\title{
Globular Clusters as Dynamical Probes of the So Galaxy NGC 3115
}

\section{JJ Kavelaars}

A thesis submitted to the Department of Physics in conformity with the requirements for the degree of Doctor of Philosophy

\author{
Queen's University \\ Kingston, Ontario, Canada \\ December, 1997
}

C)JJ Kavelaars, 1997 
Lationar Library

of Canada

Acquisitions and Bibliographic Services

395 Wellington Street Ottawa ON K1A ON4 Caneda

\section{du Canada}

Acquisitions et services bibliographiques

395, nue Wellington

Otawa ON K1A ON4

Canada
The author has granted a nonexclusive licence allowing the National Library of Canada to reproduce, loan, distribute or sell copies of this thesis in microform, paper or electronic formats.

The author retains ownership of the copyright in this thesis. Neither the thesis nor substantial extracts from it may be printed or otherwise reproduced without the author's permission.
L'auteur a accordé une licence non exclusive permettant à la Bibliothèque nationale du Canada de reproduire, prêter, distribuer ou vendre des copies de cette thèse sous la forme de microfiche/film, de reproduction sur papier ou sur format électronique.

L'auteur conserve la propriété du droit d'auteur qui protège cette thèse. Ni la thèse ni des extraits substantiels de celle-ci ne doivent être imprimés ou autrement reproduits sans son autorisation. 


\begin{abstract}
This thesis presents a photometric and spectroscopic investigation of the globular cluster system (GCS) of the S0 galaxy NGC 3115. Photometric observations, obtained at the CFHT, were made in $V$ and $I$. The limiting magnitude in both filters is approximately at the level of the peak of the globular cluster luminosity function, determined to be $m_{V}^{T O}=22.8 \pm 0.2$. This turnover value, when compared with that of the Milky Way, implies a distance modulus of $(m-M)_{V}^{T O}=30.2 \pm 0.3$ which is consistent with distance estimates based on the magnitude of the red giant branch tip. Analysis of the integrated $V-I$ colours of the clusters indicates that the GCS contains multiple population components. The red clusters appear more concentrated towards the plane of NGC 3115 than do the blue clusters.

From the spectroscopic observations, obtained at the WHT using the LDSS, we find that the cluster system is rapidly rotating and that the velocity dispersion of the cluster system implies $M / L=19_{-3}^{+10}$ at a distance of $R=15 \mathrm{kpc}$ from the center of NGC 3115 . This value of $M / L$ is larger than that found at smaller radii suggesting that NGC 3115 possesses a dark matter halo.

By combining the spectral and photometric data we find very strong evidence that NGC 3115 possesses two separate and distinct cluster populations, one a metal-rich thick disk system and the other a metal-poor halo system. This is the first detection of a disk system of clusters in a non-Local Group galaxy.
\end{abstract}




\section{Co-Authorship}

This thesis presents work conducted in collaboration with my supervisor D.A. Hanes. 


\section{Acknowledgements}

I gratefully acknowledge the support of NSERC through the operating grant of DAH, as well as that of NATO through NATO Collaborative Research Grant \#941223. Further I would like to thank Queen's University for financial support of travel necessary for the completion of this thesis. I also thank the School of Graduate studies for awarding a Thesis Completion Bursary which aided in expediting the completion of this work. I am grateful to Bill Harris of McMaster University for allowing me to take up a position at McMaster during the final stages of writing and I am grateful for the support of the McMaster Physics Department. Further I would like to thank Steve Butterworth for allowing me to use his data reduction software and for the numerous conversations and arguments we enjoyed together. In addition I am thankful to Kathy Perrett for aiding me in all aspects of the preparation of this document and for her input into its scientific content. The entire astronomy group has been a tremendously supportive during my tenure at Queen's and for that I am truly grateful. I am also greatly indebted to Dave for his inspiration, his CAP lecture in 1988 drew me into astronomy and his enthusiasm for the subject is unwavering. Thanks to Marcia for a place to sleep and SJD for a place to talk and to Andrew and Anna for food and shelter also. The list goes on and on..... 


\section{Dedication}

For Jo with all my love. You waited. You watched. You encouraged. The waiting is over. For everything you have done, for you.

\section{Statement of Originality}

For this work I conducted the observations and performed all data analysis and interpretation. The original project was motivated through discussion with my supervisor, D. A. Hanes. The observations were conducted as part of a joint observing run at the WHT and independently at the CFHT. I was present for both runs and operated the science instruments at the telescope during the acquisition of data presented in this theses. 


\section{Contents}

1 Introduction 1

1.1 Globular Clusters . . . . . . . . . . . . . . . . 3

2 Galaxy Formation and Globular Clusters 6

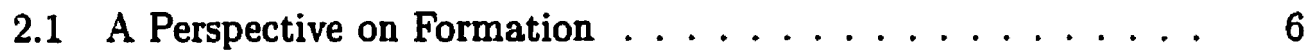

2.2 Globular Cluster Systems $\ldots \ldots \ldots \ldots \ldots$

2.2.1 The luminosity function of globulars $\ldots \ldots \ldots \ldots 8$

2.2.2 The Colour Distribution . . . . . . . . . . . 10

2.2.3 The Radial Profile . . . . . . . . . . . . 12

2.2 .4 Colour Gradient . . . . . . . . . . . . . . . 12

2.2 .5 Dynamics . . . . . . . . . . . . 13

3 Spectroscopic Techniques 15

3.1 The Evolution of Spectral Observations . . . . . . . . . 15

3.2 Multi-Object Spectroscopy . . . . . . . . . . . . 17

3.3 Two MOS Systems $\ldots \ldots \ldots \ldots \ldots \ldots \ldots$

4 The target: NGC 3115

4.1 Previous Kinematic Observations . . . . . . . . . 21

4.2 Previous Photometry . . . . . . . . . . . 23 
5 Direct Imaging $\quad 24$

5.1 Observations. . . . . . . . . . . . . . 24

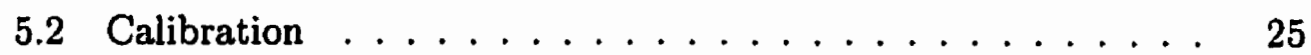

5.3 The program frames $\ldots \ldots \ldots \ldots \ldots \ldots \ldots$

5.3 .1 Preprocessing . . . . . . . . . . . . . 28

5.3.2 Photometry . . . . . . . . . . . . . . 30

5.4 Completeness . . . . . . . . . . . . . . 35

5.4 .1 The recovery curves . . . . . . . . . . . 37

6 Direct Imaging Analysis 41

6.1 The radial distribution. . . . . . . . . . . . 41

6.2 Colour . . . . . . . . . . . . . . . . . 45

6.2 .1 Metallicity . . . . . . . . . . . . . 49

6.3 The GCLF . . . . . . . . . . . . . . . . 49

6.4 Specific Frequency $\ldots \ldots \ldots \ldots \ldots \ldots \ldots$

7 Spectroscopy $\quad 57$

7.1 The Observations . . . . . . . . . . . . 57

7.2 Object Selection . . . . . . . . . . . . 58

7.3 Mask Layout . . . . . . . . . . . . . . 59

7.3.1 Preprocessing . . . . . . . . . . . . 63

7.4 Spectral Extraction . . . . . . . . . . . . 64

7.4.1 The Program Frames . . . . . . . . . . . . 64

7.4.2 Radial Velocity Standards . . . . . . . . . . 65

7.5 Velocity Calibration . . . . . . . . . . . . 66

7.6 Velocities $\ldots \ldots \ldots \ldots \ldots \ldots \ldots \ldots \ldots \ldots . \ldots . \ldots . \ldots$

7.6 .1 Cross Correlation . . . . . . . . . . . 72 
7.6 .2 Internal Dispersion $\ldots \ldots \ldots \ldots \ldots \ldots$

8 Velocity Analysis $\quad 78$

8.1 The Velocity Cut ..................... 78

8.2 Rotation ........................... 79

8.3 The Mass of NGC $3115 \ldots \ldots . \ldots . \ldots 2$

8.3.1 The Projected Mass Estimator ........... 82

8.3.2 The Mass-to-Light Ratio ............. 85

8.4 Linking colour with velocity .............. 86

8.4 .1 Rotation ................... 86

8.4 .2 The Mass .................... 88

9 Discussion and Summary $\quad 89$

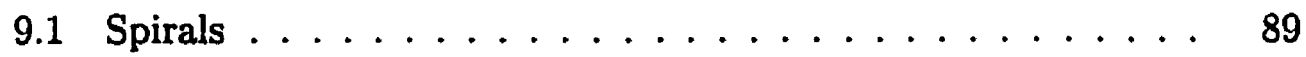

9.2 Ellipticals ........................... 90

9.3 Lenticulars . . . . . . . . . . . . . . . . . 92

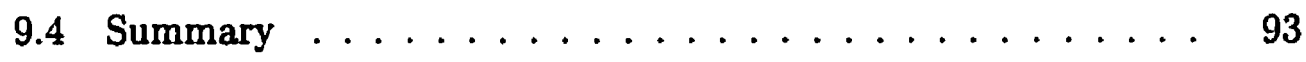

$\begin{array}{ll}\text { A Acronyms } & 99\end{array}$

B A comparison of the globular cluster luminosity functions of the inner and outer halo of the Milky Way and M31. 101

B.1 Abstract ............................. 101

B.2 Introduction . . . . . . . . . . . . . . . 102

B.3 The Milky Way GCLF, inside and out . . . . . . . . . 102

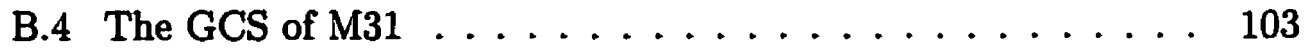

B.5 Conclusions ...................... 107

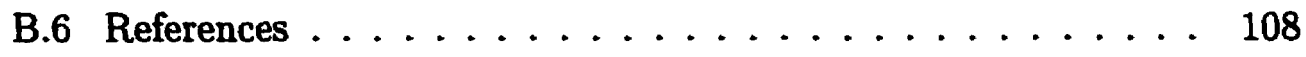


C Curriculum Vitae 


\section{List of Tables}

4.1 Physical parameters for NGC $3115 \ldots \ldots \ldots \ldots \ldots$

5.1 Observation $\log \ldots \ldots \ldots \ldots \ldots \ldots \ldots \ldots$

5.2 Calibration Coefficients . . . . . . . . . . . . 33

5.3 Aperture Corrections . . . . . . . . . . . . . . 33

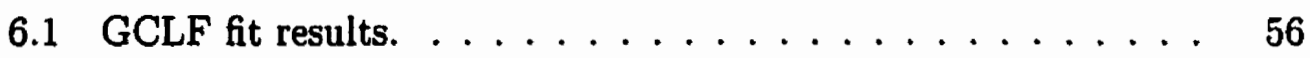

7.1 Observing $\log \ldots \ldots \ldots \ldots \ldots \ldots \ldots \ldots$

7.1 Observing $\log \ldots \ldots \ldots \ldots \ldots \ldots \ldots \ldots$

7.2 Fraunhofer lines. . . . . . . . . . . . . 69

7.3 Radial Velocity Standards . . . . . . . . . . . 71

7.4 Velocity scatter. . . . . . . . . . . . . . 74

7.5 Cross correlation velocities. $\ldots \ldots \ldots \ldots \ldots \ldots$

7.5 Cross correlation velocities. . . . . . . . . 76

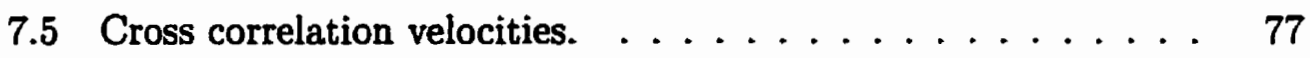

8.1 Galaxy Mass-to-Light Ratios . . . . . . . . . . . . . 87

B.1 Results of fits to the GCLF of the MW . . . . . . . . 104

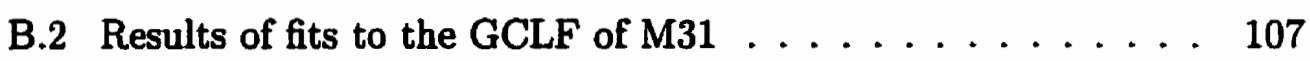




\section{List of Figures}

3.1 CFHT/MOS-SIS light train $\ldots \ldots \ldots \ldots \ldots$

5.1 The target field $\ldots \ldots \ldots \ldots \ldots \ldots \ldots \ldots \ldots \ldots$

5.2 Image classification $\ldots \ldots \ldots \ldots \ldots \ldots \ldots \ldots$

5.3 Photometric Calibration Check . . . . . . . . . . 36

5.4 Recovery Fraction, radial dependence $\ldots \ldots \ldots \ldots$

5.5 Recovery Fraction, field variations . . . . . . . . . . 40

6.1 Radial distribution of the GCS $\ldots \ldots \ldots \ldots$

$6.2 \log -\log$ radial distribution. . . . . . . . . . . . . . 44

6.3 Radial profiles and galaxy type $\ldots \ldots \ldots \ldots \ldots$

6.4 Colour distribution function $\ldots \ldots \ldots \ldots \ldots \ldots$

6.5 Colour as a function of radius $\ldots \ldots \ldots \ldots \ldots \ldots$

$6.6 \vee$ VGCLF $\ldots \ldots \ldots \ldots \ldots \ldots \ldots \ldots \ldots \ldots \ldots \ldots \ldots$

6.7 I GCLF $\ldots \ldots \ldots \ldots \ldots \ldots \ldots \ldots \ldots \ldots \ldots \ldots$

6.8 Specific Frequency $\ldots \ldots \ldots \ldots \ldots \ldots \ldots$

7.1 Slit Placement . . . . . . . . . . . . . . 61

7.2 Slit Sizes . . . . . . . . . . . . . . . 61

7.3 Spectrum of $\mathrm{M92} \ldots \ldots \ldots \ldots \ldots \ldots \ldots$

7.4 Velocity calibration .................. 70 
8.1 Velocity Histogram $\ldots \ldots \ldots \ldots \ldots$

8.2 Colour Magnitude Diagram . . . . . . . . . . . 81

8.3 Velocity and major/minor axis position . . . . . . . 83

8.4 Spatial Distribution of the GCS . . . . . . . . . . 87

B.1 MW GCLF . . . . . . . . . . . . . . . . . 104

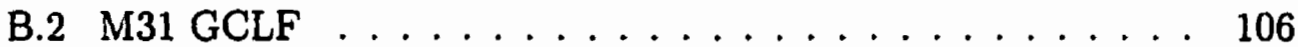




\section{Chapter 1}

\section{Introduction}

Understanding the world around us is one of the foremost goals of human civilization. Not just knowing that the Sun will rise and set, but understanding why or rather how this occurs. Science is the quest to explain and understand. Through unbiased observation of the universe, the world around us, and wellcrafted theory, astronomers have long forced society to deeper understandings of our very existence.

The occupation of astronomers as shapers of world view dates back to when people first began to look to the heavens and attempt to explain the beauty of the night sky. The stars, sentinels of the sky, transfixed and steady, draw our minds towards the heavens and push humanity to understand. From the use of stellar constellations as navigation tools to the measurement of continental drift via the positions of the most distant quasars, the sky beckons.

In exploring the world around us observation often challenges; a view of the universe that is in conflict with observation must be wrong or the incorrectness of the observations must be explained. In the 17th century the Earth was removed from is pre-eminent position as the center of all when observation showed that the Sun was in fact the center of the Earth's motion. The "per- 
fection" of circular orbits was abandoned when Kepler showed that elliptical orbits provided a simple explanation of the motions of the planets across the sky. In the 19th century, spectroscopy and Doppler's Law revealed the motions of the stars and the orbit of the Sun about the Galactic center (Mihalas and Binney 1981). In 1899 the first spectrum of a nebula was recorded and by the 1920 s these nebulae were determined to be hundreds of $\mathrm{kpc}$ from the Sun, island universes (Rubin 1995).

The evolution of our understanding has continuously pushed the role of the Earth from the center to one of insignificance. In 1933 the first evidence that visible matter may not be the dominant gravitational attractor was presented by Zwicky (1933). In 1940 further evidence from galaxy rotation curves was being found,

It may be concluded that the distribution of mass in the system must be considerably different from that of the light. A study of the light-distribution does not, therefore, enable us to draw conclusions regarding such problems as stability or the total mass of the system. (Oort 1940)

Oort had concluded that there was a necessity of large amounts of unseen matter, dark matter, to explain the rotational velocity of M31 (Rubin 1995). In that same work Oort discussed recently acquired spectra of the lenticular galaxy NGC 3115 and concluded:

There cannot be any doubt that an extension of the measures of rotation to greater distances from the nucleus would be of exceptional interest. Also our knowledge of the system could certainly be greatly advanced if it were possible to obtain spectroscopic estimates of the true mean peculiar velocities. 
Following these early studies are many investigations of the rotation curves of disk galaxies. All lead to the same conclusions: as one departs from the center of a galaxy the luminosity decays while the density of material decays more slowly (Rubin 1983 and Freeman 1996). There is a need that the majority of matter in these galaxies is dark. Further investigation of the velocity dispersion of groups of galaxies reveals that between and within them there must be vast pools of unseen material and as one travels to larger scales the implication of unseen material grows (Carlberg et al. 1996). However, arguments regarding nucleosynthesis during the early formation of universe imply that only $5-10 \%$ of this material can in fact be made of ordinary atoms (baryons) and the vast majority is likely due to some yet unknown source (Rees 1984; Mathews, Kajino, and Orito 1996), perhaps even a new form of matter. The Earth is not the center of the Universe and perhaps even the very material from which it is made, baryonic matter, is a minority of the constituents of our Universe.

\subsection{Globular Clusters}

Globular clusters are self-gravitating aggregates of some $\sim 10^{5}$ stars that orbit about the center of mass of their parent galaxies. For a review of the properties of globular cluster systems see Harris 1991. These ubiquitous objects have absolute luminosities in the range of $-11 \lessgtr M_{v} \leqslant-3$, are amongst the oldest population of their parent galaxies and the radial distribution of globulars extends to tens of kpc from their parent galaxy centers. These features combine to make globular clusters unique probes of the matter distribution at large distance (10s of kpc) from the centers of their parent galaxy. The dynamical stability of these objects has preserved a large fraction of them, preventing 
their disruption during the dynamical evolution of their parent galaxy (Murali and Weinberg 1997) and as they have now completed hundreds of orbits about the galaxy center, they are in a state of dynamical equilibrium with the galaxian potential. In their spatial and luminosity distributions they provide clues to galaxy formation. and heir spectral classification provides insight to the chemical processes and time scales of galaxy formation.

The luminosity distribution of clusters is a useful standard candle (Harris 1991) and the spatial distribution provides evidence of ancient (perhaps protogalactic) mergers (Zepf and Ashman 1993b). Our understanding of systems of clusters remains incomplete. Why does the globular cluster specific frequency correlate with galaxy type? What processes dictate the radial distributions of these objects? Why do their mass/luminosity functions follow such uniform distributions and how did these systems of objects form? To answer these questions will require a complete and accurate comparison of GCSs of galaxy of diverse types and locations. This thesis presents such a comparison for the S0, lenticular, galaxy NGC 3115.

In this thesis I present the results of photometric and spectroscopic observations of the globular clusters in orbit around the galaxy NGC 3115 . The spectra will be used to determine the kinematics of the globular cluster system thus allowing the mass of the galaxy to be inferred and then compared to the luminous component of the galaxy. The ratio of the amount of material contained in ordinary stars to that implied by the cluster system dynamics provides a measure of the importance of dark matter in this galaxy. Further, the hints, regarding the formation of globular clusters systems, contained in the kinematics will be explored. The photometric properties of the GCS of NGC 3115 will be compared to those of elliptical (E) and spiral (S) type galax- 
ies. The comparisons will focus on discerning similarities between lenticulars (S0) like NGC 3115 and S and E types.

In chapter 2 I review some relevant background to the early stages of galaxy formation and the use of globular clusters as probes of this formation. Chapter 3 provides some details of the process of multi-object spectroscopy (MOS) and compares the two MOS systems used for this project. In chapter 4 I discuss the results of previous observations of NGC 3115 , both photometric and spectroscopic. Chapter 5 outlines the photometric observations and chapter 6 contains the analysis of these observations. In chapters 7 and 8 the spectroscopic observations are presented and analyzed. Chapter 9 presents the conclusions of this thesis. 


\section{Chapter 2}

\section{Galaxy Formation and Globular Clusters}

I present here a brief review of the use of the globular cluster system (GCS) as a probe of galaxy formation. Section 2.1 sketches the setting of galaxy formation and section 2.2 outlines the process of GCS formation and how these relates to overall galaxy formation.

\subsection{A Perspective on Formation}

The standard Big Bang (SBB) model is currently the standard picture for the early evolution of the universe. The basic premise is as follows. Some $10^{10}$ years ago the universe existed with extreme densities and pressures and began to expand. During this early period fusion reactions produced a determinable level of the light elements, the primordial abundances of material. The ability of the SBB to correctly predict some of these abundances is one of the great successes of the theory. For a complete analysis of the SBB model see, for example, (Peebles 1993). 
As the early expansion continued the matter continued to cool and eventually local over-dense regions dropped out of the expanding flow and contracted to form galaxies. Many problems exist with the SBB model (the flatness problem or the horizon problem, see (Peebles 1993)) but the basic frame work provides a perspective on the use of globular clusters as tracers of galaxy formation and evolution.

Initially, the collapsing material that has dropped out of the expansion is of primordial abundance. As this material contracts, star formation occurs. If the stars are massive then they will exist for short periods of time and enrich the surrounding media during the final stages of their evolution. Thus, as objects form later and later during the formation of a galaxy they will contain larger and larger percentages of heavy elements like Fe. In standard notation the symbol $\mathrm{Fe}$ is used to represent all elements heavier than $\mathrm{H}$ and He. The amount of $\mathrm{Fe}$ to $\mathrm{H}$ is measured in units of solar abundance and presented as a logarithmic ratio.

$$
[\mathrm{Fe} / \mathrm{H}]=\log [n(\mathrm{Fe}) / n(\mathrm{H})]_{*}-\log [n(\mathrm{Fe}) / n(\mathrm{H})]_{\odot}
$$

Thus the level of enrichment that has occurred, or the metallicity, is given the notation $[\mathrm{Fe} / \mathrm{H}]$. A value of $[\mathrm{Fe} / \mathrm{H}]=0$ indicates that the object has the same "metal" abundance as the Sun while objects with $[\mathrm{Fe} / \mathrm{H}]<0$ are more "metal poor" and objects with $[\mathrm{Fe} / \mathrm{H}]>0$ are more "metal rich". As the metal content of a star is determined by the enrichment that the material, of which the star is made, has gone through, the value of $[\mathrm{Fe} / \mathrm{H}]$ is a rough tracer of time of formation. Objects with $[\mathrm{Fe} / \mathrm{H}]=-0.5$ likely formed later than objects with $[\mathrm{Fe} / \mathrm{H}]=-2.0$. Note that this is generally true but not exactly the case as enrichment depends on local conditions. For discussions of these issues see 
(Ashman, Conti, and Zepf 1995; Brodie and Huchra 1991; Jacoby, Branch, Clardullo, Davies, Harris, Pierce, Pritchet, Tonry, and Welch 1992).

If the formation process of interest is episodic then objects with distinct metallicity groupings are expected; whereas if the formation process is protracted and continuous, a smoothly varying range of metallicities is likely to be observed (Eggen, Lynden-Bell, and Sandage 1962; Searle and Zinn 1978; Zepf and Ashman 1993a; Zepf and Ashman 1993b). For the Milky Way (MW) two very distinct groups of globular clusters have been found to exist; one, the halo population, is metal poorer than the other, the disk population (Zinn and West 1984; Zinn 1985; Castellani and Melchiorri 1981; Rodgers and Paltoglou 1984). This bi-modality in metallicity is taken as evidence that the disk formed subsequent to the formation of the halo (Zinn 1985). This notion is further supported by the kinematics of the two systems. The disk population is found to be rapidly rotating while the halo population is only very slowly rotating (Zinn 1985).

\subsection{Globular Cluster Systems}

To use the GCS of NGC 3115 as pointers towards understanding the nature and formation of $\mathrm{SO}$ (lenticular in shape) galaxies and dark matter (DM) halos, we will examine some basic physical properties of the GCS and relate these to the formation and evolution of the galaxy.

\subsubsection{The luminosity function of globulars}

The globular cluster luminosity function (GCLF) is the distribution function of the luminosity of globular clusters about a parent galaxy. In a binned 
form the GCLF is the number of objects per unit magnitude. The empirical distribution function is Gaussian in shape, in log-luminosity, and generally is taken to be directly indicative of the globular cluster mass function, by assuming a constant mass to light ratio.

Numerous authors have remarked on the astounding universality of the GCLF as determined via empirical observation (for example, see Hanes 1977; Harris 1991). This universality appears to extend across diverse environments and along the entire Hubble sequence of galaxy types, although some variation of mean luminosity may exist (Fleming, Harris, Pritchet, and Hanes 1995). There are however interesting dynamical evolutionary processes being investigated using simulations which may in fact dispel this appearance of universality of shape (Murali and Weinberg 1997), and certainly some observational clues to this do exist, although the turnover magnitude appears to remain constant.

In Appendix B I compare the GCLF of the Milky Way to that of M31 in order to ensure that for the metal poor halo clusters the turnover magnitude of the GCLF is constant (at least among spirals in sparse systems). In addition I will show that the shape of GCLF may not be constant with radius and these variation may indicate evolutionary effects. However, as shown in the Appendix, these variations are not important for the comparison of the halo GCS.

This thesis will examine the shape of the GCLF of NGC 3115 in comparison with other galaxies (elliptical (E) and spiral (S) type). This comparison is made to probe the similarity of the $\mathrm{S} 0$ galaxies with the $\mathrm{E}$ and $\mathrm{S}$ galaxies in an attempt to more fully understand the nature of the S0 type. In order to compare the GCLF between various galaxies we will follow the standard 
procedure of fitting (via a maximum likelihood method) a functional form to the GCLF. This comparison allows us to search for possible dependencies of GCLF shape on galaxy type.

As a by product of the apparent stability of the peak of the GCLF, the distribution can be used as a standard candle of distance (Hanes 1977; Harris 1991; Jacoby, Branch, Clardullo, Davies, Harris, Pierce, Pritchet, Tonry, and Welch 1992), although some problems with calibration of this method do exist (Ashman, Conti, and Zepf 1995; Blakeslee and Tonry 1995). Owing to their large absolute luminosity the GCLF distance indicator can be used at very large distances.

\subsubsection{The Colour Distribution}

Photometric colours are the ratio of the luminosity of an object in one band pass to another $\left(\frac{\mathcal{L}_{V}}{\mathcal{L}_{f}}\right)$. The flux from an object in a particular band pass is typically referred to on an $\log$ scale where $V=-2.5 \log L_{V}+Z$ eroPoint, $L_{V}$ is the luminosity of the object in the band pass $(\approx 550 \mathrm{~nm} \pm 100)$ the value of ZeroPoint is determined by referring to a standard system in which the star Vega has $V=0$. Typically the ratio of flux is expressed in terms of long to short wavelength, and in magnitudes becomes $V-I$. The standard scale is defined such that the star Vega has a colour of 0 (i.e. $V-I=0, B-V=0$, etc.) Values less than 0 indicate an object which is bluer than Vega and values greater than 0 indicate an object which is redder than Vega.

The colour of a star is determined by a combination of its temperature and metallicity. As a star becomes hotter it becomes bluer, owing to the shift in the peak of the radiation spectrum. Metals within the stellar atmosphere absorb photons and then re-radiate them at longer wavelengths, thus stars 
which are metal-rich appear redder than those that are metal-poor and at the same temperature. The temperature of a star depends on its mass and stage of evolution. Very massive main sequence stars appear extremely blue, but live for only a short time in this stage before evolving and cooling. This evolution is caused by a change in the location and type of nuclear fusion which is occurring within the star, see (Novotny 1973) for a description of issues regarding colour, metallicity and age.

As globular clusters are comprised of many tens of thousands of stars, their integrated colours are comprised of the average colour of their constituents. The mass function of globular clusters is such that there are only a few very massive stars and many thousands of low-mass stars. These massive stars evolve quickly and for old cluster systems, $\sim 10^{10}$ years old, the majority of light is being produced by low-mass $\left(\sim 1 M_{\odot}\right)$ stars that have evolved to the giant branch (Fischer, Welch, Mateo, and Cote 1993). The colours of these stars are only mildly dependent on age, and as the age spread among globular clusters of a given galaxy is typically very small, colour can be taken as primarily indicative of metallicity (Ashman, Conti, and Zepf 1995).

If cluster formation is protracted then there is the possibility that the material from which later clusters form has been enriched relative to the first clusters to form. The longer the period of formation the larger the spread in the colour of the clusters. Conversely, if the formation of clusters is episodic then the expectation would be that a distribution in colours should reveal structure caused by the non-continuous enrichment that would occur (Searle and Zinn 1978; Larson 1990). As noted previously, the Milky Way cluster system contains two very distinct populations: the disk system and halo system. For the disk the median colour is $V-I=1.62$ with a spread of $\sigma_{V I}=0.37$, 
whereas the median colour and spread in the halo clusters is $V-I=1.13$ and $\sigma=0.34$ (Harris 1996b). In Section 6.2 we investigate the colour distribution of clusters about NGC 3115.

\subsubsection{The Radial Profile}

The spatial extent of material about the galactic center is an obvious indicator of compactness of the galactic material and traces the distribution of luminous material and the potential in which this material orbits. If the GCS distribution exhibits the same radial profile as the galaxy light then we might infer that the clusters share the halo formation history . A more distended GCS distribution may indicate that the GCS formed on a less compacted potential than the one the halo stars have relaxed onto, or perhaps reflect a more complex formation history for the GCS (i.e. mergers). Significantly broader systems of globulars may be indicating that the clusters themselves are more properly associated with the underlying potential well in which the galaxy formed rather than the galaxy proper. As such the radial distribution and shape of the distribution of clusters indicates the shape of the potential in which the cluster system formed (Merritt and Tremblay 1993).

\subsubsection{Colour Gradient}

The radial distribution of colour is a further tracer of the formation process (Eggen, Lynden-Bell, and Sandage 1962). A trend of objects becoming bluer with increasing galactic radius indicates that the objects at small radius formed after some enrichment had occurred, while those at larger radius formed immediately, or the environment at small radius underwent more rapid enrichment(Searle and Zinn 1978). To disentangle these effects requires more de- 
tailed analysis of highly resolved spectra of individual clusters (resolution of $1.5 \dot{A}$ with a signal-to-noise per resolution element of $\sim 50$ (Jones and Worthey 1995)), a procedure that is not possible for this data set. However, a simple model which did not allow for either age gradients or metallicity variations would be excluded by the observation of a gradient, and models that would induce such gradients will be restricted if no gradient is found.

\subsubsection{Dynamics}

Perhaps the strongest indicator of separate formation histories is dynamics. Here the spatial positions of a set of objects can be correlated with their motions, allowing an estimate of the mass of the parent galaxy and thus indicating the importance of non-luminous material. In addition, populations defined on the basis of the colour relations determined previously can now be examined for distinct dynamics, supporting or detracting from the suggestion that multiple populations exist. Systems of objects with distinct kinematics (velocity dispersion, rotation, coherent bulk motion) are unlikely to have formed together.

Rotation of the cluster system is a further indicator of the stage during which the GCS formed (Larson 1990). If the GCS of a rotating disk galaxy is not rotating then we might associate the formation of the GCS with the formation of a non-rotating halo; however, if the GCS is rotating then it is more likely to have formed with the rotating disk of the galaxy.

Clearly a wealth of pointer to and restrictions on the formation process exists within the properties of the GCS. This, coupled with their intrinsic brightness and the large population (hundreds to thousands) found around typical galaxies, make them unparalleled probes of halo structure and forma- 
tion tracers. 


\section{Chapter 3}

\section{Spectroscopic Techniques}

\subsection{The Evolution of Spectral Observations}

In his thesis Babcock (1939) acquired spectra of 4 HII regions around M31, requiring over 20 hours of integration per spectrum. This monumental work provided one of the first measures of galaxy rotation (Rubin 1995).

Since 1939 there has been a growth in the size of telescopes: Babcock's observations were made using the Lick Observatory Crossley $36 "(\approx 85 \mathrm{~cm})$ refractor while modern observatories boast $4 \mathrm{~m}$ and $8 \mathrm{~m}$ reflectors. Joined with this has been the development of charge coupled device (CCD) based imagers, which have efficiencies in the range of $80 \%-95 \%$ over a large wavelength range ( $3500-10000 \dot{A}$ ). These developments have resulted in the possibility of pursuing the spectra of objects far beyond Local Group of galaxies. However even with modern $4 m$ telescopes and CCD detectors, the acquisition of a single spectrum of a $V=20$ object requires 2 hours of integration.

How then can we provide Oort with his requested peculiar velocities? The measurement of the peculiar velocities of objects in a galaxy requires that we acyuire the spectra of individual component members and thus determine their 
radial and peculiar velocities (here the term peculiar velocity is that part of the velocity that isn't part of the bulk motion of the galaxy and isn't part of the motion of a group of objects about the galaxy's center). At the distance of NGC 3115 (some $10 \mathrm{Mpc}$ ) the brightest stars $\left(m_{I} \gtrsim 25.5\right.$ (Elson 1997)) are too faint to permit the acquisition of spectral velocities; but the globular clusters of this galaxy, for which we might expect the brightest ones at $m_{I} \gtrsim 19.5$, are attainable, with sufficient integration time, using a $4 m$ class telescope. As such the cluster population is an excellent pool of test particles with which to measure the potential well of a galaxy and thus deduce the importance of dark matter in the halos of remote galaxies.

The use of globulars as probes of galaxy structure has been successfully employed in a number of circumstances. Among the first uses was the determination of the spatial extent of the Galaxy (Shapley 1918). Determining distances to these objects and assuming they lie in a roughly spherical distribution about the Galaxy center, Shapley determined the distance of the Sun from the Galactic center and a measure of the spatial extent of the Galaxy (Fernie 1962; Harris 1976). Their use as probes of the Milky Way is further extended by studying their spectra to determine their radial velocities and metallicity (Brodie and Hanes 1986; Zinn 1985). Careful examination and correlation of these quantities provides insight to the early stages of galaxy formation, see for example (Searle and Zinn 1978; Larson 1990). Models that do not account for the dynamical signature of these objects can not be correct. Early use of the spectra of globular clusters systems in probing external galaxy potentials include M31 (Andromeda) (Van Den Bergh 1969), M87 (the giant elliptical in the Virgo cluster) (Hanes and Brodie 1986; Mould, Oke, and Nemec 1987), and M49 (Mould, Oke, De Zeeuw, and Nemec 1990). These early efforts em- 
ployed single slit spectroscopy and as such required many pointings, each of considerable integration $(\sim 2 h r s)$.

\subsection{Multi-Object Spectroscopy}

Recently the development of multi-object-spectrographs (MOS) has provided an invaluable tool for the thorough investigation of the velocities of clusters about remote galaxies. At the time of writing, studies of the velocities of globular clusters about non-Local Group galaxies have been performed for a handful of galaxies (M87, M104, NGC 5128, NGC 1399, M49 and M81), each time revealing more information about the cluster system and parent galaxy. This thesis presents an answer to Oort's request (some 57 years later) by compiling radial velocity measurements for the individual members of the GCS of NGC 3115 .

The multi-object-spectrograph allows the acquisition of some dozens of spectra from objects across the detector's field of view (typically 10'). Thus, although an individual spectrum still requires two hours of observing to acquire it is possible to use the same telescope to simultaneously acquire dozens of spectra. During a single observing session, some hundreds of spectra can be acquired. Figure 3.1 shows the basic layout of the multi-object spectrograph (MOS) in use at the Canada-France-Hawaii Telescope (CFHT). The basic operating premise of the device is to place a grated-prism (grism) in the light path between the collimating lens of the detector and the CCD, thus dispersing the image in wavelength. By placing a mask in the optical path, prior to the grism, the light striking the grism can be restricted. Only the objects whose positions correspond with slits cut through the opaque mask will be imaged onto the spectrograph. For this system to be useful requires a high degree of 
accuracy in the placement of mask slits.

\subsection{Two MOS Systems}

The data for this thesis were acquired at two separate telescopes employing two different approaches to the acquisition of spectra from multiple objects in a single pointing. The main difference between these systems is in the design of object masks.

In the CFHT+MOS system, the mask is designed at the telescope, during the run, based on images acquired (usually on the first night of an observing session) through the MOS system. The slit placement is exact and the design of the mask is a dynamic process: new masks can be designed, manufactured and inserted into the telescope in less than two hours. This allows the observer to adjust the mask design with immediate knowledge of the telescope seeing (clarity of the image), and also allows the observer to first acquire images and partially analyze them in order to choose optimal candidates. This process is highly efficient and allows a very high rate of successful, high precision spectra. The William Herschel Telescope (WHT)+low dispersion survey spectrograph (LDSS) system requires that the masks be designed prior to the observing run, thus the masks are made to allow observations to take place over a large range of seeing conditions. The ability to rapidly manufacture and exchange masks in the CFHT+MOS system allows the observer to adapt to changing seeing conditions and thus maximize the throughput and resolution of the system. 


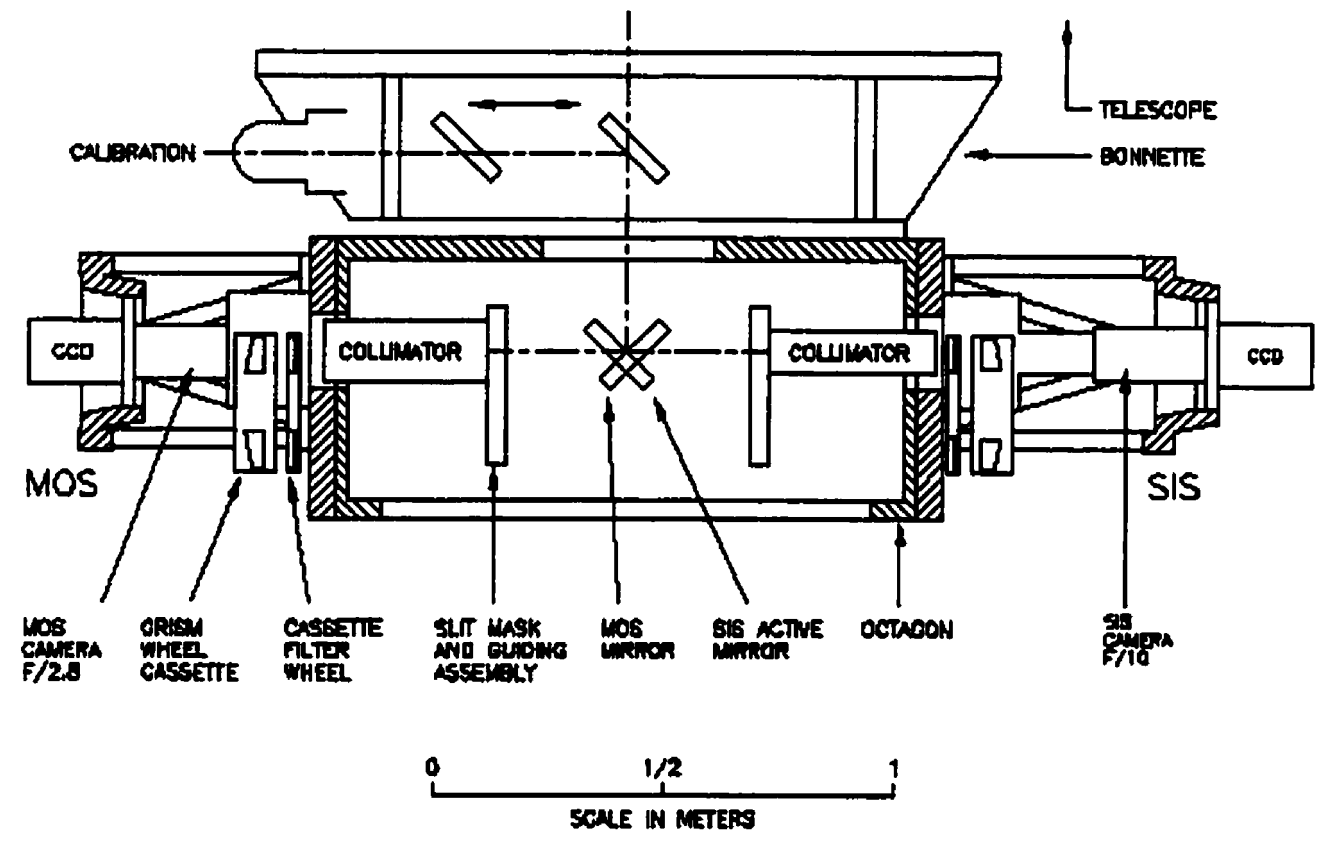

Figure 3.1 The MOS-SIS light train. Taken from the CFHT MOS manual. 


\section{Chapter 4}

\section{The target: NGC 3115}

The S0 galaxy NGC 3115 resides in the Leo spur, a low density environment. As such the encounter history of this galaxy is likely to have been relatively simple, providing an excellent laboratory for investigations of galaxy kinematics. This galaxy contains little, if any, gas, and a rapidly rotating bulge with a rotation curve that is flat out to $8.5 \mathrm{kpc}$ from the nucleus (Capaccioli, Cappellaro, Held, and Vietri 1993). Previous investigations of NGC 3115 have revealed strong evidence for $\mathrm{a} \sim 10^{9} M_{\odot}$ (Kormendy et al. 1996) black hole, a bi-modal distribution in colour of the red giant population (Elson 1997) and a disk with a very small scale height ( 1.5kpc ) (Capaccioli, Vietri, and Held 1988), although it does appear that the disk flares out at large galactic radii. This evidence for episodic formation makes NGC 3115 an interesting target for analysis of its GCS. Previous photometric studies of this galaxy have relied on photographic plates and a more accurate analysis using CCD imaging is warranted. In addition, the advent of multi-object spectrograph systems makes a complete photometric and spectroscopic study of the GCS feasible.

NGC 3115's role as an archetypical S0 galaxy of relative proximity and its nearly edge-on position make it an ideal target for numerous astronomi- 
cal investigations. There have been no fewer then 180 publications dealing specifically with observations of this interesting target. Table 4.1 provides a summary of the relevant physical parameters of NGC 3115.

\subsection{Previous Kinematic Observations}

NGC 3115 is one of the first galaxies for which disk rotation curves were measured (Oort 1940) and has been the subject of numerous investigations since (see for example (Morton and Chevalier 1973; Rubin, Peterson, and Ford 1980; Davies 1981; Walker 1989; Capaccioli, Cappellaro, Held, and Vietri 1993))

Previous studies of the kinematics of NGC 3115 have concentrated on the nuclear region of this interesting S0 galaxy. Spectra along the major axis have been used to explore the velocity field of NGC 3115. Illingworth and Schechter 1982 find that this galaxy has a rapidly rotating bulge; indeed, the rotation is almost sufficient to explain the flattened figure of this galaxy. The major axis rotation curve is flat to $\sim 100$ arc seconds radius $(\sim 5 \mathrm{kpc}$ for a distance of $10 \mathrm{Mpc}$ ), with an amplitude of $\sim 300 \mathrm{~km} / \mathrm{sec}$; the implication is that there is a considerable dark halo. Such rotationally supported flattening is characteristic of "disky" elliptical galaxies (Nieto and Bender 1989) and this may indicate that this S0 galaxy represents an extension of the E galaxy type towards that of $\mathrm{S}$ type galaxies, arguing for a smooth transition between $\mathrm{E}$ and $S$ type galaxies.

High resolution spectroscopy reveals a steep increase in the radial velocity dispersion with decreasing distance form the galaxy center, providing one of the strongest cases of highly compacted matter (i.e., a "black hole") (Kormendy and Richstone 1992). No information on rotation or velocity dispersion exists 
TABLE 4.1. Physical parameters for NGC 3115

\begin{tabular}{ll}
\hline \multicolumn{1}{c}{ Parameter } & \multicolumn{1}{c}{ Value } \\
\hline Right Ascension (1950.0) & $10^{h} 02^{m} 44.51$ \\
Declination (1950.0) & $-07^{\circ} 28^{\prime} 30^{3} .7$ \\
Morphological type & $\mathrm{S}^{-}(\mathrm{sp})$ \\
Isophotal diameter & $8^{\prime} \cdot 63 \pm 0^{\prime} .27$ \\
Axis ratio & $0.15 \pm 0.03$ \\
Position Angle & $45.5 \pm 1^{\circ}$ \\
Corrected Total Magnitude $\left(B_{T}^{0}\right)$ & $9.65 \pm 0.1$ \\
Radial Velocity & $663 \mathrm{~km} / \mathrm{s}$ \\
Distance & $10.8 \pm 0.5 \mathrm{Mpc}$ \\
\hline \hline
\end{tabular}

Notes to Table 4.1.

All values are from (de Vaucouleurs, de Vaucouleurs, Crowin Jr, Buta, Paturel, and Fouque 1991) except distance (Elson 1997) and radial velocity (Capaccioli, Cappellaro, Held, and Vietri 1993). 
beyond $\sim 8 \mathrm{kpc}$ as the disk of the galaxy is only about $20 \%$ the background level beyond this radius and so spectral analysis has been restricted.

\subsection{Previous Photometry}

Of particular relevance for this work are the previous analyses of the GCS and the light profile of the galaxy.

Previous photometric studies of NGC 3115 have revealed that the isophotes are less flattened than the isochromes (Strom, Strom, Jensen, Moller, Thompson, and Thuan 1977), an indication that some chemical evolution occurred during the galaxy's formation. Also, detailed decomposition of the light profile of NGC 3115 reveals that the underlying disk contributes less than $10 \%$ of the light of the galaxy with a scale height of 25.5 (Capaccioli, Vietri, and Held 1988) ( 1.5kpc for a distance of $10 \mathrm{Mpc}$ ). This weak disk has been interpreted as further evidence of NGC 3115 elliptical-like nature, (Capaccioli, Cappellaro, Held, and Vietri 1993).

The first study of the GCS of NGC 3115 (Strom, Strom, Jensen, Moller, Thompson, and Thuan 1977) used the counts of objects around the galaxy to determine a crude GCLF distance to this galaxy by comparing the shape of the observed GCLF with that of M31. A subsequent, more thorough study (Hanes and Harris 1986) of the GCS determined both the luminosity function of objects and analyzed their radial distribution. This more thorough analysis revealed a slight flattening of the GCS, consistent with the isophotal flattening of the galactic bulge. The broader shape of the GCLF and moderate specific frequency of clusters about the galaxy are suggestive of the GCS of an E galaxy rather than that of a spiral (for a discussion of the possible differences between elliptical and spiral GCSs see Fleming, Harris, Pritchet, and Hanes 1995). 


\section{Chapter 5}

\section{Direct Imaging}

This chapter contains an outline of the data reduction procedures used in determining the photometry of the direct images acquired at the CFHT as part of this investigation of the GCS of NGC 3115. Section 5.2 describes the standard star transformation. In Section 5.3 the method used to distinguish stellar from non-stellar objects is presented and Section 5.4 presents the method used to characterize the detection threshold of the images.

\subsection{Observations}

This projected was attempted on three observing runs at the CFHT (during the winters of 1993, 1994 and 1995). The first attempt was aborted during a ice storm at the observatory, the second lost to technical problems with the CFHT MOS system combined with poor weather. During the third run direct images of four fields around NGC 3115 were acquired at the CFHT using the MOS in direct imaging mode. Each field was imaged in two passbands $V$ and $I$, see Table 5.1. The primary purpose of these exposures was the construction of masks for the acquisition of spectra of the GCS of NGC 3115 and also to 
allow a photometric analysis of the GCS of the galaxy. However, due to the low quantum efficiency (QE) of the CCD that was available, we were forced to acquire the spectra at red $(\sim 8000 \dot{A})$ wavelengths. At these long wavelengths the very strong emission spectrum of the night sky swamped the faint absorption spectra of the globulars, thus rendering the spectral observations useless. The direct images were arranged, see Figure 5.1, to provide spatial coverage to a radius of $\sim 17 \mathrm{kpc}$ from the galaxy center (assuming a distance of $10 \mathrm{Mpc}$

). This provides a large spatial coverage allowing for a thorough photometric investigation of the GCS. Table 5.1 summarizes the program exposures and gives the field identification that will be used. The exposure times were selected to provide a signal-to-noise ratio of $\sim 5$ at the turnover of the GCLF. A value of $S / N \sim 5$ represents approximately the point at which $50 \%$ of the objects of the signal level will be detected and is the faintness limit below which little useful data can be extracted.

\subsection{Calibration}

To calibrate the photometry obtained for the program fields we observed a standard field calibrated to the $U B V(R I)_{K C}$ system ( $K C==$ Kron Cousins $\mathrm{KC}$ ). For this project we observed M 67 (a well calibrated Milky Way cluster) at multiple times throughout each night. Using these observations the following transformation equations were fit to the standard star instrumental magnitudes,

$$
\begin{array}{r}
v_{\text {inst }}-V=\alpha_{V}+\left(\beta_{V} \times X\right)+\gamma_{V} \times(V-I) \\
\left(v_{\text {inst }}-i_{\text {inst }}\right)=\alpha_{V-I}+\left(\beta_{(V-I)} \times X\right)+\gamma_{(V-I)} \times(V-I) .
\end{array}
$$

In the above equation; $\alpha$ is the ZeroPoint of the detector, $X$ represents the 
TABLE 5.1. Observation Log

\begin{tabular}{|c|c|c|c|c|c|}
\hline Target & RA & DEC & Airmass & Filter & Exp. Time \\
\hline M 67 & $8: 48: 35.9$ & $11: 57: 32$ & 1.244 & I & $2 s$ \\
\hline M 67 & $8: 48: 36.0$ & $11: 57: 32$ & 1.237 & I & $2 s$ \\
\hline M 67 & $8: 48: 35.9$ & $11: 57: 32$ & 1.232 & V & $5 \mathrm{~s}$ \\
\hline M 67 & $8: 48: 36.0$ & $11: 57: 33$ & 1.226 & V & $2 \mathrm{~s}$ \\
\hline M 67 & $8: 48: 36.0$ & $11: 57: 32$ & 1.221 & V & $2 \mathrm{~s}$ \\
\hline North & 10:03:51.9 & $-7: 33: 44$ & 1.62 & $\mathrm{~V}$ & $100 s+2 \times 900 s$ \\
\hline North & $10: 03: 51.9$ & $-7: 33: 44$ & 1.40 & I & $100 s+900 s$ \\
\hline South & 10:04:09.5 & $-7: 38: 10$ & 1.35 & I & $100 s+900 s$ \\
\hline South & 10:04:09.5 & $-7: 38: 10$ & 1.25 & V & $100 s+2 \times 900 s$ \\
\hline East & 10:04:07.8 & $-7: 33: 28$ & 1.16 & V & $2 \times 900 \mathrm{~s}$ \\
\hline East & $10: 04: 07.8$ & $-7: 33: 28$ & 1.13 & I & $900 \mathrm{~s}$ \\
\hline West & $10: 03: 51.5$ & $-7: 37: 34$ & 1.14 & I & $900 \mathrm{~s}$ \\
\hline West & $10: 03: 51.5$ & $-7: 37: 34$ & 1.15 & $\mathrm{~V}$ & $2 \times 900 s$ \\
\hline M 67 & $8: 48: 35.9$ & $11: 57: 32$ & 1.354 & V & $2 \mathrm{~s}$ \\
\hline M 67 & $8: 48: 36.0$ & $11: 57: 31$ & 1.346 & $\mathrm{~V}$ & $1 \mathrm{~s}$ \\
\hline M 67 & $8: 48: 35.9$ & 11:57:32 & 1.337 & I & 1s \\
\hline M 67 & $8: 48: 35.8$ & $11: 57: 32$ & 1.330 & I & $0.5 \mathrm{~s}$ \\
\hline M 67 & $8: 48: 36.2$ & $11: 57: 35$ & 1.718 & V & $2 . s$ \\
\hline M 67 & $8: 48: 36.1$ & $11: 57: 36$ & 1.733 & I & $0.5 \mathrm{~s}$ \\
\hline M 67 & $8: 48: 36.2$ & $11: 57: 38$ & 1.750 & I & $0.5 \mathrm{~s}$ \\
\hline M 67 & $8: 48: 36.2$ & $11: 57: 39$ & 1.764 & V & $1 \mathrm{~s}$ \\
\hline M 67 & $8: 48: 36.5$ & $11: 57: 31$ & 1.611 & I & $2 \mathrm{~s}$ \\
\hline M 67 & $8: 48: 36.5$ & $11: 57: 31$ & 1.598 & I & $2 \mathrm{~s}$ \\
\hline M 67 & $8: 48: 36.6$ & $11: 57: 31$ & 1.586 & V & $3 s$ \\
\hline M 67 & $8: 48: 36.6$ & $11: 57: 31$ & 1.574 & $\mathrm{~V}$ & $3 s$ \\
\hline
\end{tabular}

Notes to Table 5.1.

North, South, East, West targets are the NGC 3115 fields. Where multiple exposures of the same field were later combined only one entry is shown. The airmass quoted in the table is the average value during the exposure. 


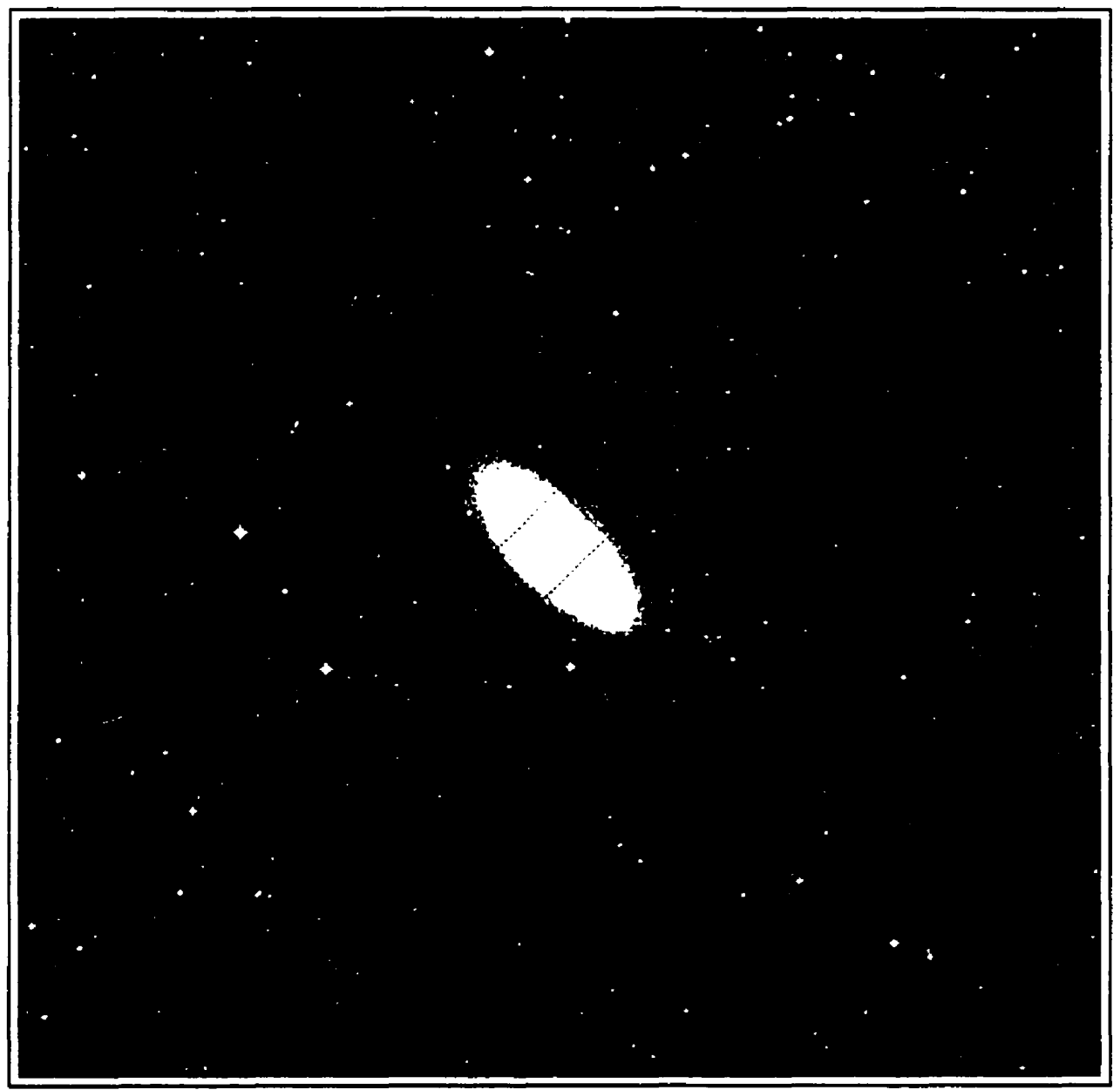

Figure 5.1 A Digitized Sky Survey image showing the area imaged for this study of the GCS of NGC 3115, North is directly up and East directly right. The boxes are $10^{\prime} \times 10^{\prime}$ and represent the area covered by single pointings of the MOS imager. The boxes are labeled according to the designations used in the text. 
airmass and $\beta$ the extinction term, results from the scattering and absorption of light by the atmosphere. The determination of the coefficients was done using the noao. photcal package in IRAF ${ }^{\mathfrak{l}}$ and the standard magnitudes used were those of (Porter, Davis, and Hall 1990). The coefficients are given in Table 5.2

\subsection{The program frames}

\subsubsection{Preprocessing}

Before CCD data can be analyzed it is necessary to remove the signature of the detector from the data set. This step is referred to as "pre-processing" as it occurs before the data is actually processed to provide astronomical information.

Within a CCD each pixel acts as a well in which electrons are trapped after having been photo-ionized from the semiconductor substrate from which the device is made. In order to use the data the number of electrons in each pixel well is read using an analogue to digital converter (ADC). This process introduces into the data a read-noise in addition to the Poisson shot-noise of the actual photon detection process. The read-noise introduced into the data by $A D C$ is a function of the bias level in the CCD array. Thus, to minimize the read-noise the ADC introduces a preset bias into the data. The bias reduces the read-noise of the system. The preset bias level is, in principle and by design, very stable over time scales of a few nights observing and can be characterized

\footnotetext{
${ }^{1}$ IRAF: Image Reduction and Analysis Facility is distributed by the National Optical Astronomy Observatories (NOAO), which is operated by the Association of Universities for Research in Astronomy, Inc. (AURA) under cooperative agreement with the National Science Foundation (NSF).
} 
by reading the CCD without exposing the chip. These frames are referred to as "zero" or "bias" frames and typically 10 or so frames are acquired per night to provide an accurate measure of the preset level. In addition to these bias frames, the level is further characterized by allowing the ADC to continue to read-out the CCD after all signal from detected photons has been wiped from the device. This "over-clocked" region is then attached to the recorded data as a measure of the bias level for the current readout.

To remove the bias level from the CCD data is then a two step process. First the over-clock strip is examined to determine the current bias level for the entire chip, a zeroth order determination of the bias. As the bias level varies, in a consistent way, from pixel to pixel the over-clock strip is only used to remove the bulk bias level from the dataset, including the actual bias frames. After the local over-clock is removed from each of the individual bias frames these frames are combined together be replacing each pixel with the median value of that pixel in each of the separate frames, referred to as a "median combined image". This provides a high-accuracy low-noise measure of the pixel-pixel variations in the bias level. This final zero frame is then subtracted from the over-clock corrected program frames, removing from them the bias level that was added by the ADC.

After correction for the bias level the image is corrected for the non-uniform illumination of the $\mathrm{CCD}$ and for the non-uniform sensitivity of the $\mathrm{CCD}$, "flat fielding". This is achieved by taking exposures of the bright twilight sky. A series of offset images are combined to provide a blank image (any stars in the individual images are rejected during the combining of the multiple exposures) and this image is then divided by its mean count level. This provides an image which is near unity everywhere with deviations that are due to spatial 
variations in the illumination of the detector and differences in sensitivity of neighboring pixels. This "flat" image is then divided into each of the program frames to remove the detection sensitivity pattern from the data. Analysis of blank sky frames reveals that the achieved flattening was of order $2.5 \%$.

\subsubsection{Photometry}

To extract photometry of the stellar images in the CCD frames the daophot task was used. There are two general approaches that can be followed in determining the luminosity of images in the CCD frame. If the background sky level is smooth, and the field is not crowded, then one can use aperture photometry. This procedure involves placing apertures (circular apertures in the case of daophot) centered on the stellar images and measuring the total flux through the aperture. Then a second, larger, aperture is used and the flux in the annulus between the inner and outer apertures is measured, the flux through this annulus is then used to determine the sky level. The outer annular flux is then scaled to an area equal to that of the inner aperture and the difference of the inner flux to the scaled outer annulus is the flux from the stellar object of interest.

If the background varies rapidly or the stellar images are close together then this method will not work. In these cases profile fitting or point-spreadfunction (PSF) photometry is required. The procedure for PSF photometry and the technique of median filtering is well established, (Stetson 1987; Fischer, Hesser, Harris, and Bothun 1990; Perrett, Hanes, Butterworth, Kavelaars, Geisler, and Harris 1997). The following is a summary of the basic procedure. For PSF photometry the user defines a PSF for each frame based on bright isolated stellar images in the frame. The magnitudes of these isolated bright 
objects are determined via aperture photometry and this sets the zero point of the magnitude scale of the PSF photometry. Then at each location in the frame where a stellar image is detected the PSF is scaled to best match the stellar image. The flux that the fit stellar image contains is then subtracted from the CCD frame. Once all the stellar objects have been removed the frame should only contain background light. This background light is then modeled (in this case we replace each pixel in the frame with the median of all pixels, within a box 5 seeing profiles wide, centered on the pixel) and the modeled light is subtracted from the original CCD frame. The process of finding stellar images and fitting them with a PSF is then repeated on this new median filtered frame. As the frame has been smoothed with a median filter the sky is smoothly varying. For these observations of the GCS of NGC 3115 the field was not crowded and so the background level can now be determined using aperture photometry on the median filtered images.

At the distance of NGC 3115 ( 10Mpc ) images of a typical Milky Way globular cluster, with core radius of $\sim 5 \mathrm{pc}$, would subtend an angle of $\sim 0.1^{\prime \prime}$, about 10 times smaller than the seeing disk of these observations. Thus images of our target objects, the globulars of NGC 3115, are indistinguishable from stellar images. However, even at much larger distances images of background galaxies are still resolvable. Using image classification, these resolved background objects can be culled from the dataset, and thus removed as a contaminant from the overall analysis. The photometry for individual objects has been determined by fitting a PSF to stellar like sources using the the task noao.digiphot.daophot.allstar which returns a "goodness of fit" parameter CHI. Figure 5.2 shows a plot of this "goodness" statistic for a set of artificial data, which were generated using the noao.artdata package and the 
PSF from West program field in the $I$ band. Clearly stellar images all have values of $C H I<1.6$. This same parameter for the West program field $I$ data is also shown and indicates the culling used. This same culling parameter was used for all four program fields and each filter.

Visual inspection of the frames reveals that objects which have been rejected via this CHI test are obviously non-stellar and that the majority of objects that appear non-stellar in the frame are rejected via the CHI test.

An aperture of 4 pixels radius was used to determine the magnitudes of the objects in the program frames. This aperture size was used as it was found to maximize the signal-to-noise ratio for the fainter objects in the field. However variations in seeing and changes in the telescope focus cause the percentage of the total light which lands in this small aperture to change from frame to frame. To compensate for this variation, the magnitudes of a few bright stars were determined using both the small aperture and a larger aperture that contained all the light from the star. The difference in the magnitude of a star in these two apertures is the aperture correction. This shift is applied to magnitudes calculated for the fainter stars for which only the smaller aperture was used. The stars in the calibration frames are all bright objects and as such the total sky flux is small compared to that of the star and therefore only the larger aperture is used on these frames. The aperture corrections were determined using the photcal package and are listed in Table 5.3

The photometry of the culled dataset was then transformed to the standard system using the coefficients in Table 5.2. For objects which had no matching photometry in the opposing filter (ie objects for which only $I$ or $V$ photometery was returned by allstar) the colour of the object was fixed at $V-I=1.0$. This is necessary to allow an estimate of the colour terms in Equations 5.1. 
TABLE 5.2. Calibration Coefficients

\begin{tabular}{ccc}
\hline \hline V-Coef. & Value & Uncertainty \\
\hline$\alpha_{V}$ & 0.22 & 0.02 \\
$\beta_{V}$ & 0.12 & 0.01 \\
$\gamma_{V}$ & -0.01 & 0.01 \\
$\alpha_{(}(V-I)$ & -0.04 & 0.04 \\
$\beta_{(V-I)}$ & 0.00 & 0.03 \\
$\gamma_{(}(V-I)$ & 1.04 & 0.03 \\
RMS-error & \pm 0.02 & \\
RMS-error $V-I$ & \pm 0.04 & \\
\hline \hline
\end{tabular}

Notes to Table 5.2.

Transformation equation coefficients for the CFHT-MOS direct imaging data. The coefficients are described in Equations 5.1.

TABLE 5.3. Aperture Corrections

\begin{tabular}{ccccc}
\hline \hline Field & $V$ correction & $\sigma_{V}$ & Icorrection & $\sigma_{I}$ \\
\hline North & -0.31 & 0.02 & -0.62 & 0.03 \\
South & -0.72 & 0.03 & -0.62 & 0.02 \\
East & -0.85 & 0.04 & -0.72 & 0.02 \\
West & -0.61 & 0.03 & -0.43 & 0.03 \\
\hline \hline
\end{tabular}



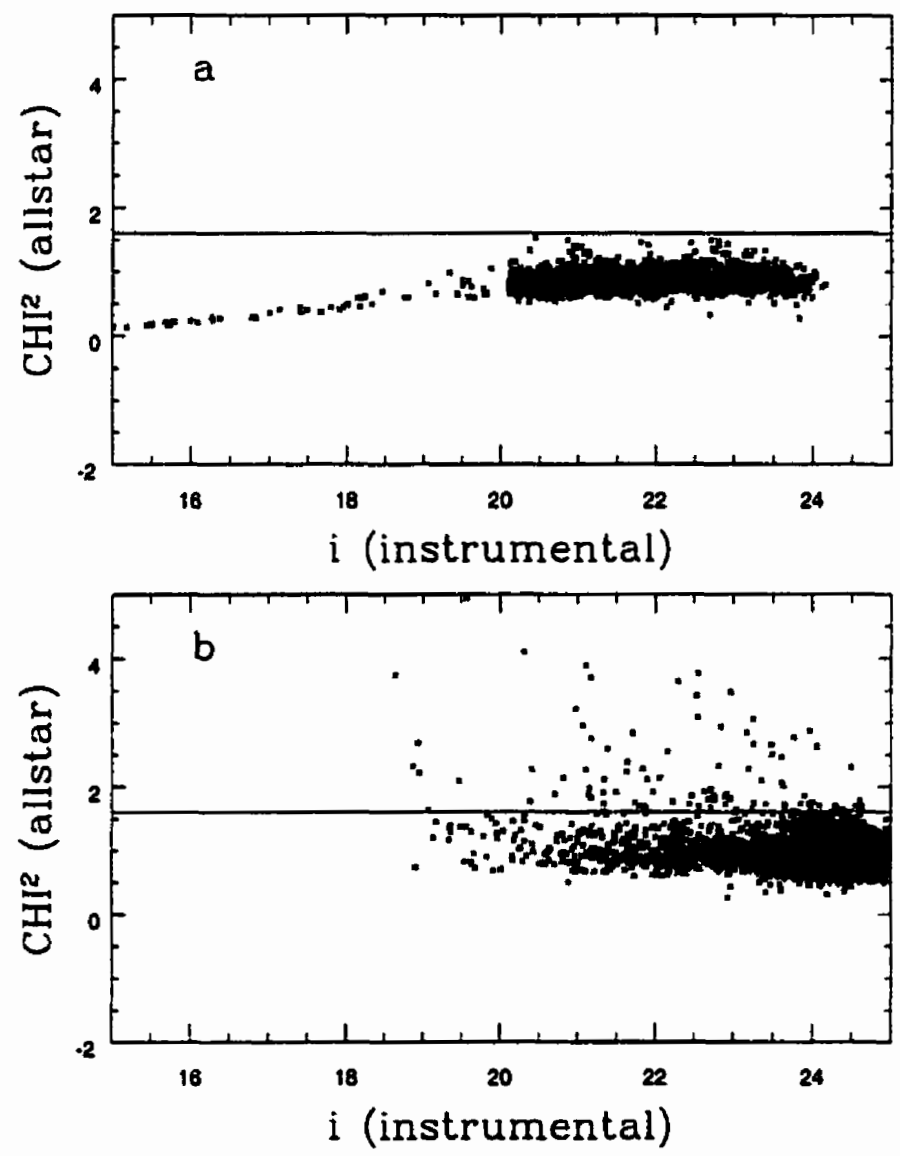

Figure 5.2 The "goodness of fit" $\mathrm{CHI}$ parameter as a function of magnitude for a) artificially generated data and b) the West I filter data. Objects for which CHI exceed 1.6 are considered likely to be non-stellar and have been eliminated from the final dataset. Also, objects fainter than the $50 \%$ recovery level are not used in the analysis, this level occurs at $i_{(i n s t r u m e n t a l)} \sim 23.5$. 
$V-I=1.0$ was the average value for the objects for which the colour could be determined and so was used in this estimate.

Objects that were detected in the West frame in $V$ and $I$, and each of the other frames in turn, were then compared to check the consistency of the photometry between the frames, see Figure 5.3. There is no strong evidence for a systematic shift between the various frames except for the North frame. The North photometry appears to have a slight scale error as a function of magnitude in $V$ and $I$, however the focus was poorest for this frame and as the scatter is generally within $2 \sigma$ of zero, no adjustment to the North photometry was made. In the region of interest for this data, $21<V<23$, the mean offset between the frames is not different from zero.

\subsection{Completeness}

In order to determine the luminosity distribution of objects down to the faintest objects detected, the data must be corrected for incompleteness. The "incompleteness"' of an image is the percentage of objects which are missed by a given reduction algorithm and is typically presented as a function of object brightness. For typical CCD+IRAF/noao.digiphot.daophot reductions the completeness of an observation is $100 \%$ for bright objects and decays rapidly to zero starting approximately 1 magnitude brighter than detection limit of the data ( $0 \%$ completeness), see Figure 5.5.

To determine the shape of the completeness curve artificial stellar data are added to each frame so that the number of objects found can be compared to the number that were added. The completeness function is a binomial distribution and as such the uncertainty in a given completeness value is given 

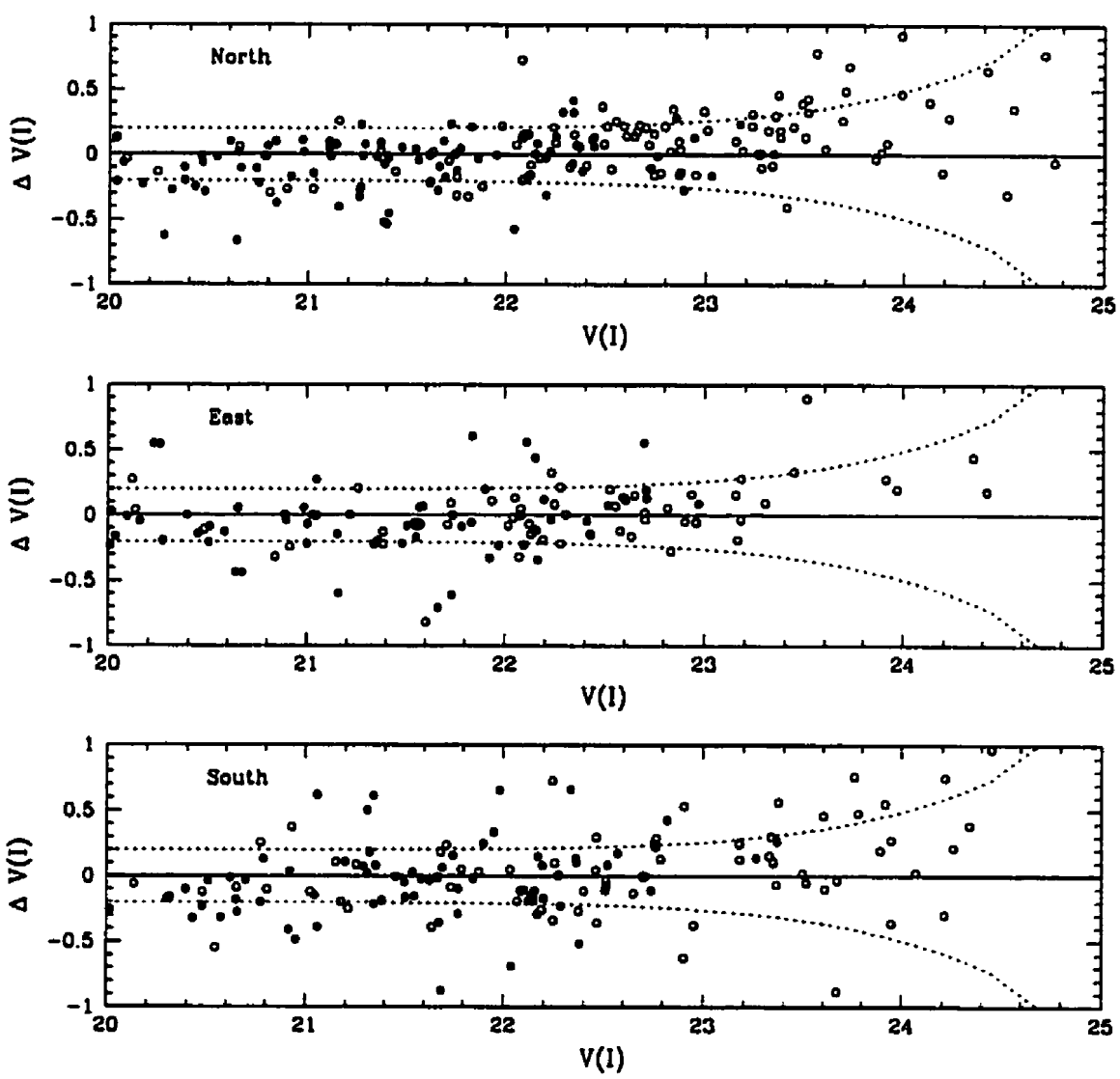

Figure 5.3 A comparison of the photometry resulting from the four different pointings around NGC 3115. $\Delta$ values are in the sense of West - North, West - East and West - South. The open circles are for $V$ and the filled circles are for $I$. The dashed line represents the average $2 \sigma_{V}$ error and includes the Poisson and calibration errors for both pointings (West+North, West+East, West+South) added in quadrature. 
by

$$
\sigma_{\mathrm{i}}=\frac{f_{\mathrm{i}} *\left(1-f_{\mathrm{i}}\right)}{\operatorname{NADD}_{\mathrm{i}}}
$$

where $f_{\mathfrak{i}}$ is the completeness value for stars whose brightness ranges between $\left(\mathrm{m}_{\mathrm{i}}-w / 2, \mathrm{~m}_{\mathrm{i}}+w / 2\right)\left(w\right.$ is the bin width) and $\mathrm{NADD}_{\mathrm{i}}$ is the number of stars added to the $i_{t h}$ bin. This formulation of the error assumes that the magnitude at which an object is found is the same as that at which it was "added" to the frame. This is, of course, not always the case. However, large numbers of simulations indicate (Butterworth 1997) that there is no significant difference between the completeness curve as determined by this assumption and one which is more correctly based on the re-binning of objects based on magnitudes at which they are discovered. The only real difference between these two approaches is in the number of artificial star simulations required to measure the completeness to the desired accuracy. For small simulation sets some small difference between the two approaches was found to exist for values of completeness below 0.3 but data at levels of completeness below 0.4 are not used in our analysis of the GCS and so those differences can be ignored.

\subsubsection{The recovery curves}

For each of the program frames a recovery-fraction curve was determined by the following procedure:

- Using the IRAF package noao.digiphot.addstar 500 stellar profiles were added to the image. The stellar profiles were derived from the PSF image combined with random noise characteristics of the program frame. The profiles were distributed randomly within the frame and randomly between the saturation and detection thresholds of the frame. 
- The artificial frames were analyzed in exactly the same manner as the original frames (excluding the creation of a new PSF image).

- The resulting allstar output was classified according to the image classification described above.

- The input star list was matched against the list of objects found in the frame. Objects that didn't match the spatial position of the input list to within 0.5 pixels were ignored.

- The preceding steps were repeated ten times per frame per filter.

- The input artificial star list and found artificial star list were binned based on magnitude, and the ratio of stars added to the frame to stars found by daophot was determined. This allows determination of the completeness fraction at any magnitude to within the resolution set by the bin widths.

The completeness levels of all four fields were found to be statistically identical, see Figure 5.5. This is expected as all the data were acquired on the same night and the seeing conditions did not very significantly. After masking off all data interior to a circle of 75 pixels raidus centered on the galaxy the completeness level was found to be independent of chip position, see Figure 5.4. From this analysis the completeness limit of the data (the point at which fewer than $50 \%$ of objects present are detected) was determined to be $m_{V(I)}=23.2(22.1)$. 


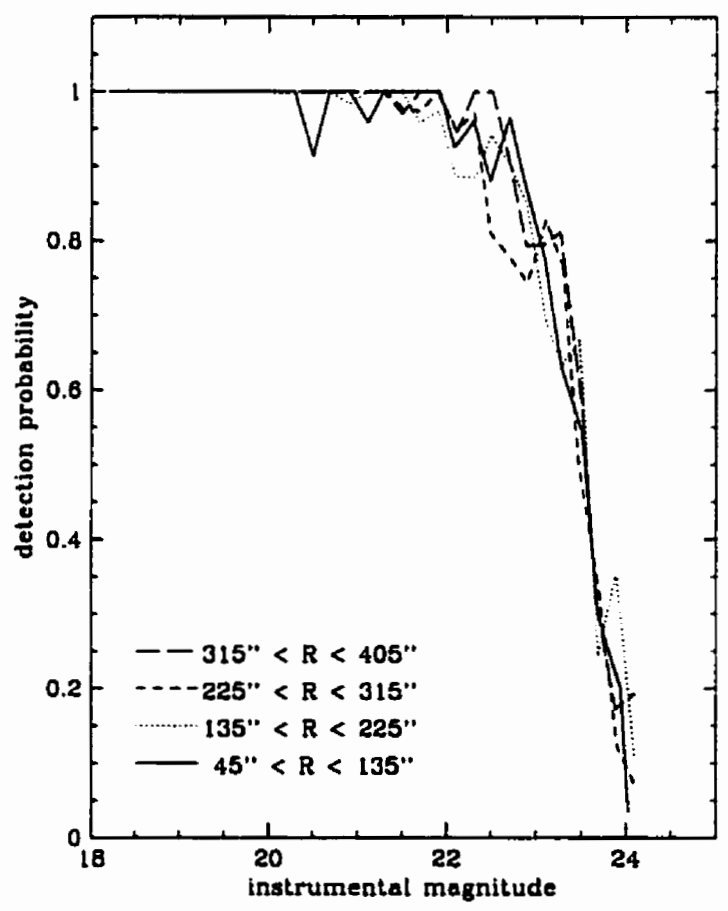

Figure 5.4 The recovery curve for the South $I$ frame in instrumental magnitudes. The recovery fraction does not depend on galactic radius, $R$. 


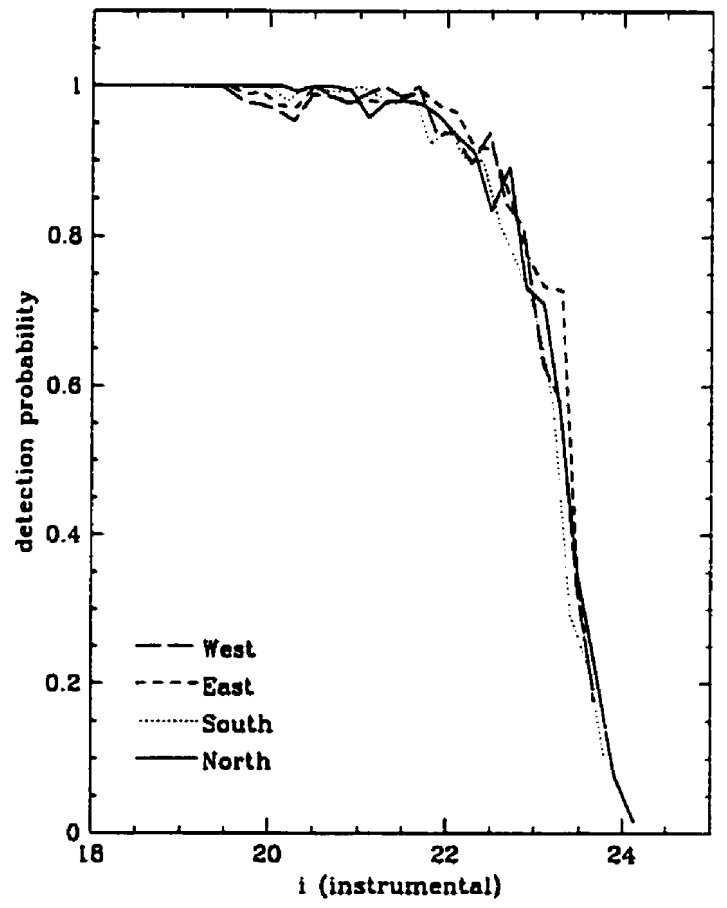

Figure 5.5 A comparison of the recovery fraction curves for each field. The recovery curve does not differ between frames. 


\section{Chapter 6}

\section{Direct Imaging Analysis}

In this chapter I present the results of the analysis of the spatial and luminosity distribution of objects about the galaxy NGC 3115. In searching for clues to the origins and evolution of cluster systems, four major features of the GCS are examined: the spatial distribution, the colour, the luminosity, and the total population (the specific frequency, $S_{N}$ ) as a function of parent galaxy luminosity.

Section 6.1 contains the analysis of the radial distribution of the cluster system, section 6.2 examines the colour distribution of objects, and section 6.3 presents the luminosity function of objects about the galaxy. Section 6.4 contains the determination of the specific frequency of the GCS.

\subsection{The radial distribution.}

The radial distribution of objects about NGC 3115 was determined using the $V$ dataset which extends about 0.2 magnitudes fainter than the $I$ data. Section 6.2 contains an examination of the colour distribution as a function of radius. 
To determine the radial distribution of the objects, each frame's dataset was treated separately and then averaged after binning. Each dataset was binned into radial annuli of $0.5^{\prime}$ width with corrections being made for incompleteness of the data at faint magnitudes. The range of magnitudes used was restricted to $20<V<23.5$ to prevent contamination from foreground stars and ensure that no objects fainter than the $50 \%$ completeness (see Figure 6.6 ) level were used. Figure 6.1 shows the radial distribution of all objects which meet this criterion.

From Figure 6.1 the distribution appears flat beyond a radius of 5 arcminutes (a linear distance of $\sim 15 \mathrm{kpc}$ for a distance of $10 \mathrm{Mpc}$ ), this is consistent with this area of the distribution being dominated by background galaxies and foreground stars, as expected at large distances from the center of the galaxy. To determine the background level the density of objects in the bins between $5.5^{\prime}$ and $7.5^{\prime}$ was averaged and this average value was then subtracted from each bin. A plot of the background-subtracted density profile is shown in Figure 6.2. A power-law, $\log (\rho(R))=\alpha * \log (R)+C$, least-squares fit to this distribution gives $\alpha=-1.8 \pm 0.2, \chi^{2} \sim 0.1$. The quoted uncertainty is the formal value of the least-squares fit and does not reflect uncertainty in the background level. If the background level is actually $10 \%$ higher than that found, then $\alpha=-2.4$, while if the level is $10 \%$ lower then $\alpha=-1.4$. Thus a value of $\alpha=-1.8 \pm 0.5$ is reasonable.

The coefficient of a power law fit of cluster surface density as a function of radius is seen to correlate well with luminosity (size) of the parent galaxy (Harris 1986). Figure 6.3 shows a plot of power index $(\alpha)$ as a function of parent galaxy luminosity. NGC 3115 is represented by the large filled square 


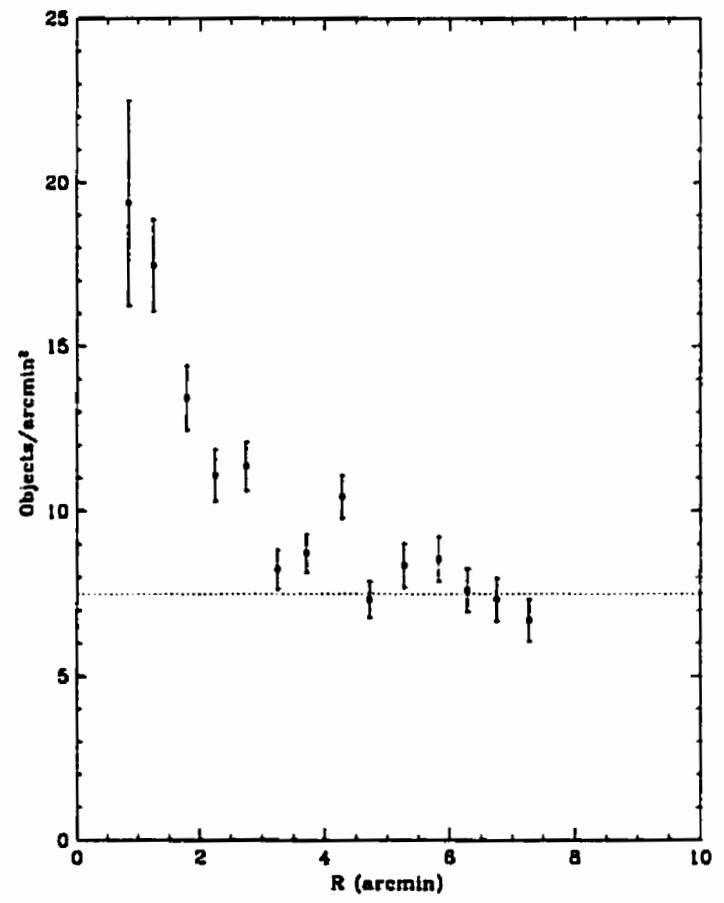

Figure 6.1 Radial distribution of the surface density for all objects for which $20<V<23.5$. The dashed line is the estimate of the background level based on the 4 outermost bins. 


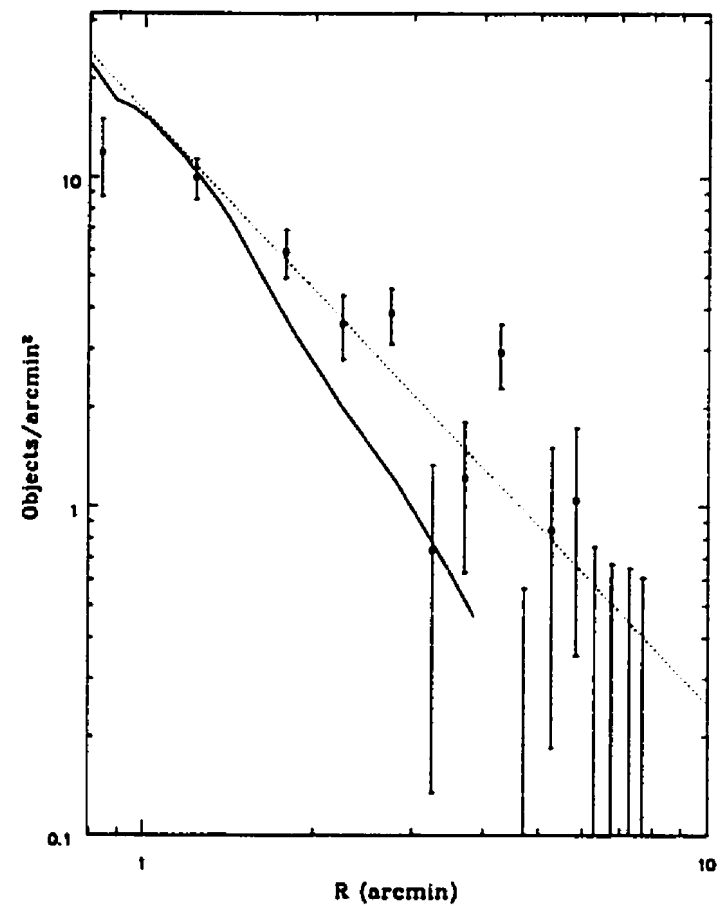

Figure 6.2 Radial distribution of the surface density for all objects for which $20<V<23.5$ plotted on a $\log$-log scale. On this scaling the power-law relation is linear. The result of a fit of a power-law $(\alpha=-1.8)$ to the data is shown by the dashed line. The solid line indicates the light profile of the galaxy bulge. 
in this figure and is seen to lie near the line of best fit for this relation. The correlation of the spatial size of the cluster population with galaxy luminosity is consistent with the more luminous galaxies being more massive and thus possessing a GCS with a larger spatial distribution. The strength of the correlation indicates that a galaxy's GCS is associated with the galaxy's potential.

\subsection{Colour}

For objects that were detected in both the $V$ and $I$ bandpasses, the ratio of the flux in $V$ to $I$ bandpasses, the $V-I$ colour, could be determined. To examine the colour distribution of the GCS of NGC 3115 we must first select those objects that are likely to be clusters. Likely clusters were selected as those objects for which $21.5<V<22.5$ and $1.0^{\prime}<R_{G C}<3.0^{\prime}$. In addition a background colour distribution was determined by selecting objects in the same magnitude range and $6.0^{\prime}<R_{G C}<7.5^{\prime}$. Both the inner and outer distribution were then binned and their difference reveals the colour distribution of globular clusters about NGC 3115, see Figure 6.4. This distribution was then fit with a three-component Gaussian and a one-component Gaussian. The three-component fit is clearly superior; the GCS of NGC 3115 is multimodal in colour. The central peaks of the distribution occur at $V-I=0.56$ $V-I=0.99$ and $V-I=1.29$. The reduced-CHI parameter for the uni-modal fit rejects the fit at the $99 \%$ level $(\chi=8.67)$ while the multi-modal fit is accepted $(\chi=0.67)$. Reduced $\chi$ values near $1(1.5$ to 0.5$)$ imply a good fit while large values reject the fit.

The mean $V-I$ colour as a function of radius is presented in Figure 6.5. As the figure indicates, there is no trend of colour as a function of radius. 


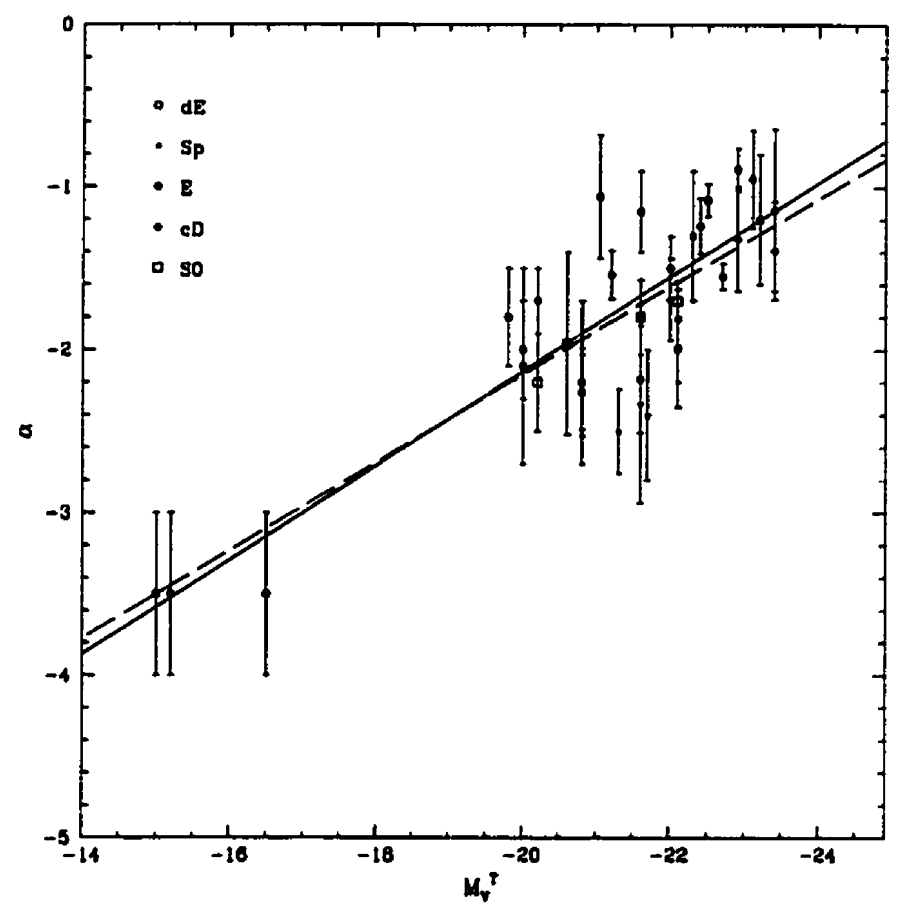

Figure 6.3 Index of power-law fit to the radial distribution of globular cluster density shown as a function of the parent galaxy luminosity. The solid line is a fit to all the data points and the dashed line does not include the $\mathrm{dE}$ data points. The large filled square is the result obtained for NGC 3115 (formal uncertainty only). Three of the four measures of $\alpha$ for $\mathrm{S} 0$ galaxies lie below the average relation. Although there is a great deal of scatter in the relation the values of $\alpha$ for the S0 galaxies suggests that the GCSs of S0 galaxies are more compact than the GCSs of E galaxies of similar luminosity. All values from (Perrett, Hanes, Butterworth, Kavelaars, Geisler, and Harris 1997) except NGC3115. 


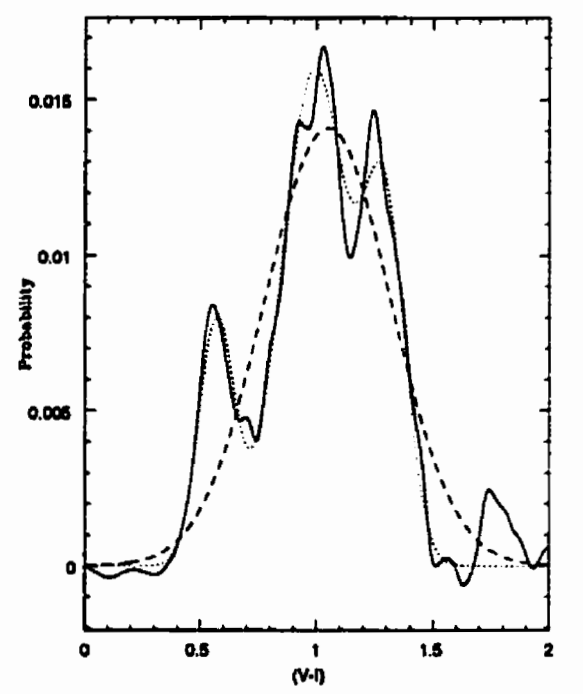

Figure 6.4 The background subtracted $V-I$ density distribution for objects selected as likely globulars, see text for details of selection. The dotted line is a fit of multi-modal Gaussian and the solid line is a uni-modal fit. The uni-modal fit is rejected at the $99 \%$ confidence level 


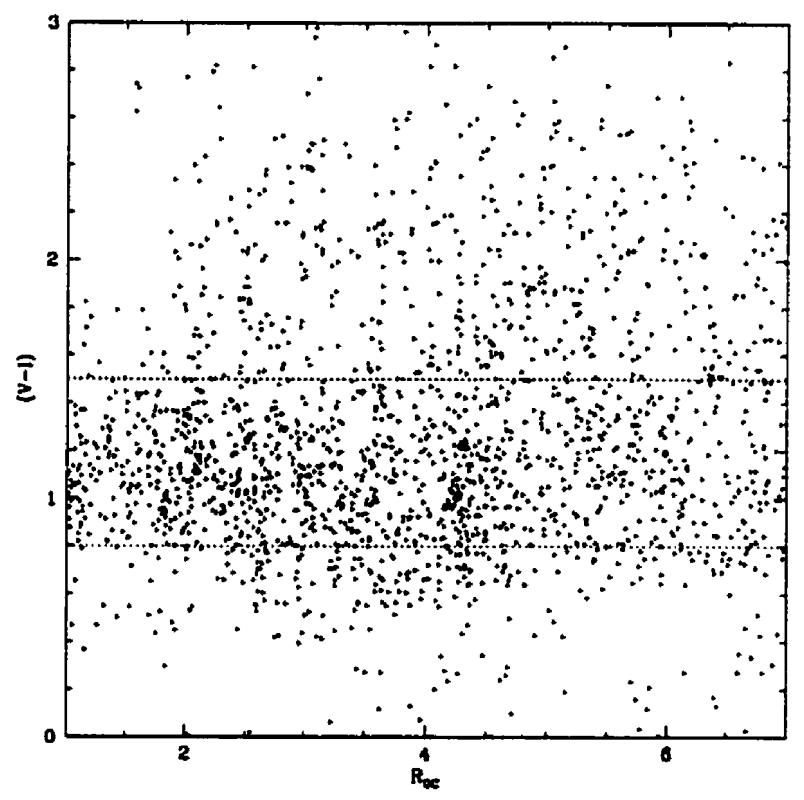

Figure 6.5 The $V-I$ colour as a function of the projected distance of objects from the center of NGC 3115. There appears to be no trend of $V-I$ with radial position. The dashed lines indicate the approximate limits of the cluster population as determined by an inspection of Figure 6.4 . 


\subsubsection{Metallicity}

For the globular clusters of the Milky Way the disk population has a mean colour of $\langle V-I\rangle=1.62$ and a mean metallicity of $\langle[\mathrm{Fe} / \mathrm{H}]\rangle=-0.55$ while for the halo clusters $\langle V-I\rangle=1.13$ and $\langle[\mathrm{Fe} / \mathrm{H}]\rangle=-1.57$. For the metal poor halo clusters of the Milky Way,

$$
(V-I)_{0}=0.15[\mathrm{Fe} / \mathrm{H}]+1.20
$$

(Harris 1996b).

The above relation is only an indicator of metallicity and may seriously over estimate metallicities for objects with $[\mathrm{Fe} / \mathrm{H}]>-0.5$ (Harris, Geisler, Harris, and Hesser 1992). Based on this relation between colour and metallicity, and the colour in each of the peaks found in the colour distribution of NGC 3115 (Figure 6.4) a metallicity of $[\mathrm{Fe} / \mathrm{H}]=-1.17$ for the halo (blue) and $[\mathrm{Fe} / \mathrm{H}]=0.5$ for the disk (red) systems is found. Clearly there has been an phase of enrichment subsequent to the formation of the halo GCS of NGC 3115 , or the halo system has been captured from a more metal-poor system.

The red-giant halo stars of NGC 3115 exhibit a bi-modal colour distribution with peaks at $(V-I) \sim 1.75$ and $(V-I) \sim 2.25$ (Elson 1997). Thus the halo globulars are bluer (more metal poor) than both system of halo stars, a finding that is typical of halo GCSs (Harris 1991).

\subsection{The GCLF}

The luminosity function of clusters about a parent galaxy is found empirically to follow that of a normal distribution, and the width and peak value of the GCLF are remarkably stable across galaxy type and environment (for example (Harris 1991; Hanes and Whittaker 1987)). The stability of form 
of the GCLF and ubiquity of globular clusters is a strong constraint in theories of the formation and evolution of GCSs. More recently the use of the t5 distribution function has evolved to replace the Gaussian as the choice of fitting function in analyzing the shape of the GCLF for galaxies with sparse (fewer then 1000 objects) cluster populations (Secker and Harris 1993; Secker 1992; Abraham and van den Bergh 1995), although the resulting widths of the function are still quoted in Gaussian widths where the width of an equivalent Gaussian is 1.29 times the $t 5$ width. A number of authors have commented on and shown that to best determine the shape of the underlying distribution of objects which creates the observed GCLF, a maximum-likelihood approach should be employed (Hanes and Whittaker 1987; Secker and Harris 1993). A "package" algorithm for such a determination is now available in the form of the MAXIMUM program (Secker and Harris 1993).

In determining the shape of the luminosity function an accurate background is critical. The level of foreground stellar density rises exponentially and can obscure the true shape of the GCLF; at faint magnitudes, $V>22$, background galaxies become a strong contaminant. The density level of these noise signals varies spatially on scales of a few arc-minutes and so a background area that is near to the target galaxy is crucial. From Figure 6.1 we see that the cluster population is essentially zero beyond a radius of $\sim 5^{\prime}$ from the galaxy center. Thus we can use the data beyond this radius to measure the background level of objects. We thus define the background from the region $6^{\prime}<R<7.5^{\prime}$. This region is well away from the bulk of the cluster distribution and avoids areas at large radii from the galaxy center which are invariably imaged on the edge of the CCD and are subject to vignetting and thus a brighter limiting magnitude than the center of the field. 
To determine the shape of the GCLF, a large sample of clusters is required. To achieve this goal the data from all four frames has been combined. The zero points of these four samples are consistent and an average recovery curve was used to correct for completeness. To determine the best parameters for modeling the data the code MAXIMUM is used to fit the $t 5$ function,

$$
\gamma(m)=\frac{8}{3 \sqrt{5} \pi \sigma_{t}}\left(1+\frac{\left(m-m^{0}\right)^{2}}{5 \sigma_{i}^{2}}\right)-3
$$

where $\sigma_{t}$ is the width of distribution and $m^{0}$ is the turn-over or peak of the distribution. Figures $6.6 \& 6.7$ shows the results of the maximum-likelihood fit. The width and turnover magnitude of these fits are given in Table 6.1. The $V$ turnover magnitude of $m_{\text {TO }}^{V}=22.8 \pm .2$ combined with a distance modulus of $(m-M)=30.2 \pm .1$ implies that the absolute value of the turn-over occurs at $M_{T O}^{V}=-7.4 \pm .25$, typical of spiral galaxies (Fleming, Harris, Pritchet, and Hanes 1995). In addition the width of the distribution, $\sigma_{t 5} \sim 0.8 \pm 0.05$, is similar to that found for the spiral M31, $\sigma_{t 5}=0.82 \pm 0.01$ (Secker 1992)

\subsection{Specific Frequency}

The magnitude limit of this investigation is $V=23.2$ (the $50 \%$ completeness limit, see Figure 6.6). Thus, from the fit of the GCLF we have observed $~ 60 \%$ of the luminosity function of NGC 3115. Taking the radial distribution and multiplying the density of objects in each radial bin by the area of the bin and subtracting off the previously found background density results in a total of 465 objects brighter than the completeness threshold of the system, for a total population of 775 clusters. To determine the total population of objects requires knowing the density of objects in the innermost radial bin. This inner bin was exclude from the fit in Figure 6.2 as its value is likely suppressed 

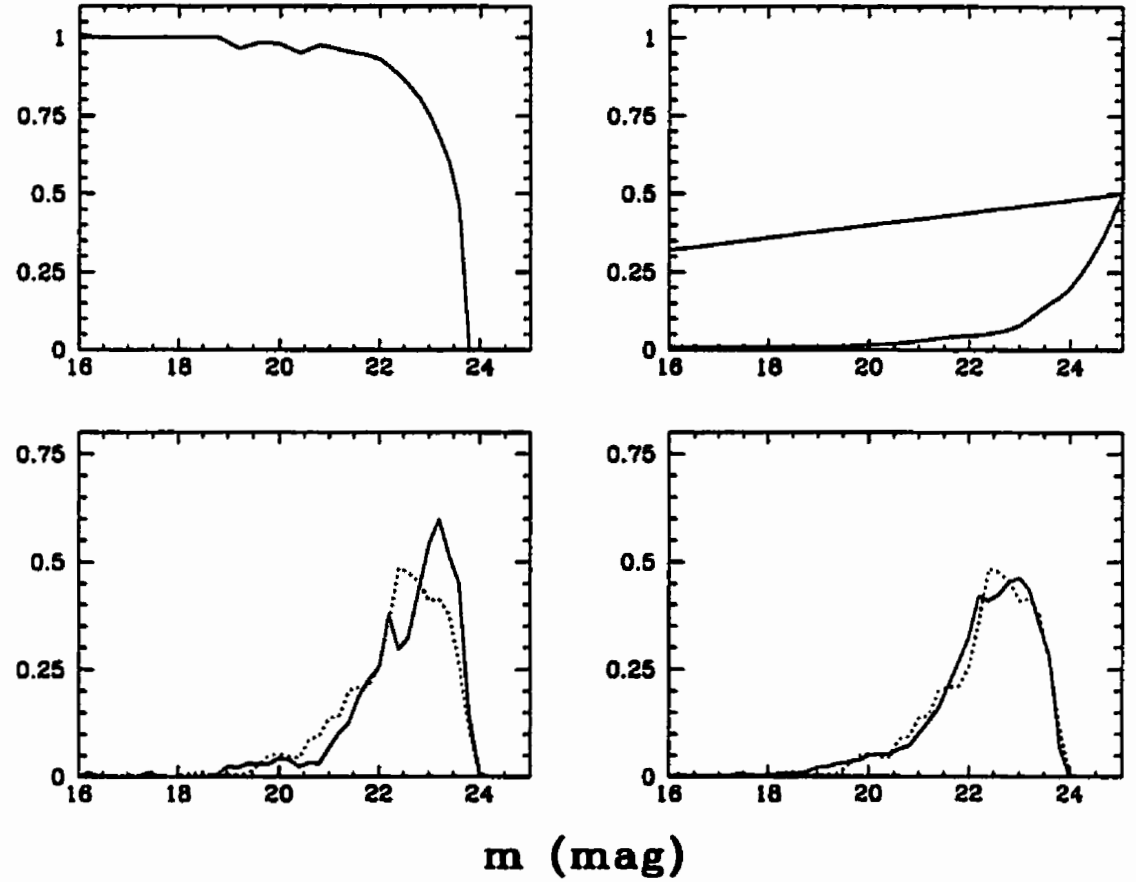

Figure 6.6 Results of MAXIMUM for the V GCLF. Counterclockwise starting with the upper right these plots are i) the photometric error, ii) the completeness, iii) the background LF (solid line) and the observed LF of objects around NGC 3115 (dashed line) iv) the maximum-likelihood solution for the combined background LF and a GCLF with a t5 distribution (solid line) and the observed LF (dashed line). All plots are probability per magnitude versus $V$ magnitude. 

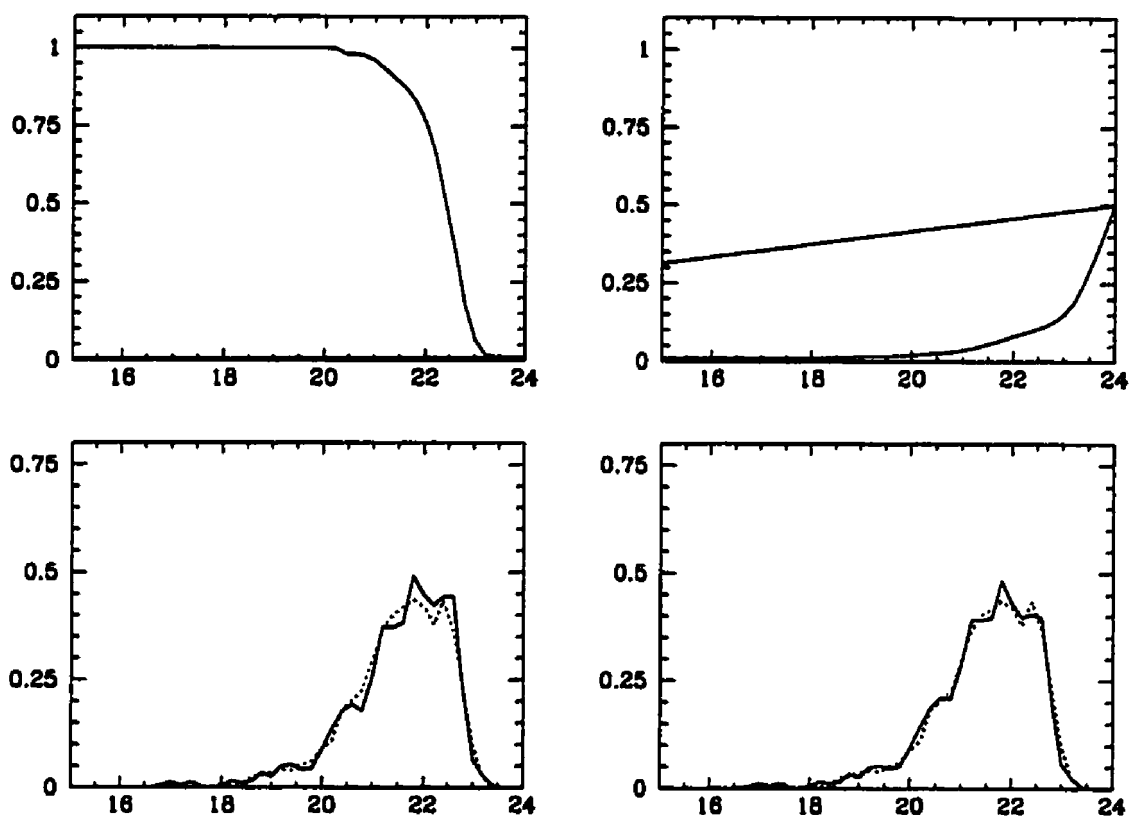

m (mag)

Figure 6.7 Results of MAXIMUM for the I GCLF. Counterclockwise starting with the upper right these plots are i) the photometric error, ii) the completeness,iii) the background LF (solid line) and the observed LF of objects around NGC 3115 (dashed line) iv) the maximum-likelihood solution for the combined background LF and a GCLF with a t5 distribution (solid line) and the observed LF (dashed line). All plots are probability per magnitude versus $I$ magnitude. 
by incompleteness effects very near the galaxy center. Thus, the inner density (11 clusters/arcminute) is determined from the power-law fit and not the inner data point.

The specific frequency of a GCS is a measure of the total number of clusters per unit luminosity of the parent galaxy (Harris and Van Den Bergh 1981).

$$
S_{N}=N G C \times 10^{0.4\left(M_{V}^{T}+15.0\right)}
$$

Where $N G C$ is the total number of globular clusters around the galaxy. This relation removes the dependency on parent galaxy luminosity from the total number of globulars and allows a comparison of total relative populations of clusters of different galaxies.

We find that for NGC $3115 S_{N}=2 \pm 1$. This result assumes a distance modulus of $(m-M)=30.2$ and $m_{T}^{V}=8.75$ (de Vaucouleurs, de Vaucouleurs, Crowin Jr, Buta, Paturel, and Fouque 1991). Figure 6.8 compares the value found for NGC 3115 with those of other E, S0 and Spiral galaxies.

Of interest in Figure 6.8 is the, albeit weak, trend of $S_{N}$ with luminosity in the S0 type. The low luminosity S0 have low values of $S_{N}$, typical of Sa and $\mathrm{Sb}$ galaxies while the brighter $\mathrm{S} 0$ galaxies are more like $\mathrm{E}$ types in their value of $S_{N}$. This apparent shift in association of So from spirals to ellipticals is consistent with other parameters (the luminosity function S0, their isophotal shapes, the prominence of bulge components, etc.) of these interesting objects (van den Bergh 1990; van den Bergh 1997). 


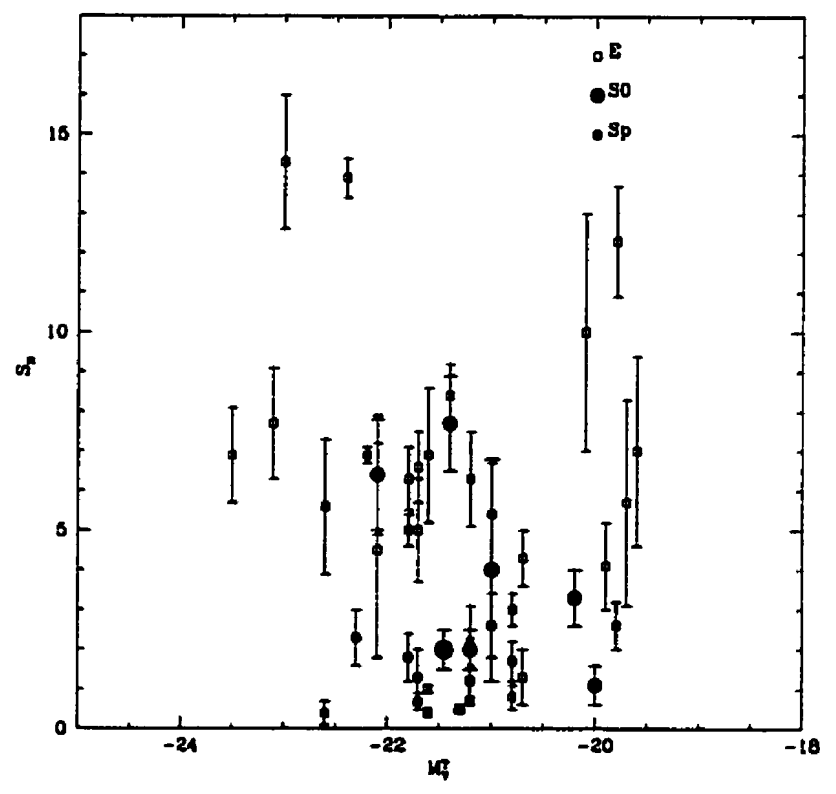

Figure 6.8 Specific frequency as a function of parent galaxy luminosity. All data taken from the McMaster Catalogue (Harris 1996a), except the NGC 3115 point (large filled circle) which is taken from this work. Spirals all have a value of $S_{N}<3$ while the $\mathrm{E}$ galaxies span the range from 0 to 15 . The two lenticular galaxies with values of $S_{N}$ larger than five both contain central bars and are type SB0. 


\section{TABLE 6.1. GCLF fit results.}

\begin{tabular}{lcccc}
\hline \multicolumn{1}{c}{ Data set } & $m_{T O}^{V}$ & $\sigma_{t 5}$ & $m_{T O}^{I}$ & $\sigma_{t 5}$ \\
\hline All data & $22.8 \pm 0.1$ & $0.81 \pm 0.05$ & $21.6 \pm 0.2$ & $0.75 \pm 0.08$ \\
$0.80<(V-I)<1.50$ & $22.7 \pm 0.1$ & $0.81 \pm 0.08$ & $21.9 \pm 0.2$ & $0.77 \pm 0.08$ \\
$0.80<(V-I)<1.15$ & $22.6 \pm 0.2$ & $0.71 \pm 0.08$ & $21.9 \pm 0.4$ & $0.71 \pm 0.08$ \\
$1.15<(V-I)<1.50$ & $23.0 \pm 0.2$ & $0.86 \pm 0.11$ & $21.9 \pm 0.2$ & $0.91 \pm 0.13$ \\
\hline \hline
\end{tabular}

Notes to Table 6.1.

A comparison of GCLF fit parameters determined for various subsets of the GCS of NGC 3115. There are no significant differences between parameters as determined using the various subsets. All values were determined via maximum likelihood analysis and $1 \sigma$ errors are quoted. 


\section{Chapter 7}

\section{Spectroscopy}

In this chapter I discuss the acquisition of the spectra of target globulars about NGC 3115 . Section 7.1 outlines the actual observations and data preprocessing. Section 7.2 details the selection of target objects that are likely to be globular clusters. Section 7.3 describes the process of creating a mask for use in a multi-object-spectrograph. The extraction of the actual spectra from the CCD images and their calibration are reviewed in sections 7.4 and 7.5. Section 7.6 presents the final velocities found for all objects observed.

\subsection{The Observations}

The observations for this work were carried out at the $4 \mathrm{~m}$ William Herschel Telescope (WHT) using the Low Dispersion Spectrograph (LDSS) in "Blue Arm" mode. The LDSS has a field of view of $10^{\prime} \times 10^{\prime}$ and spectral resolution of $2.5 \dot{\mathrm{A}} /$ pixel from $3500-5000 \dot{\mathrm{A}}$. The observing log appears as Table 7.1.

In the LDSS system the slit mask is designed prior to arrival at the summit using astrometry of the target field. We were able to acquire direct images of NGC 3115 at the Cerro Tololo Inter-American Observatory (CTIO) prior 
to the run at WHT for use in construction of the mask for LDSS. The CTIO images were acquired under poor seeing conditions and are not adequate for a photometric analysis but did provide good astrometric positions. The frames taken using the CFHT+MOS system, during the 1st and 2nd run at CFHT, are of even poorer quality. In addition the CFHT+MOS system introduces large amounts of image distortion and so construction of a LDSS mask from those data was not appropriate. As the masks for the LDSS were constructed prior to the observing session, we were forced to plan for the most probable conditions and so created masks with slits that were large enough for poor to average conditions. These masks were made with $1.5^{\prime \prime}$ slits and so the resolution of the spectra acquired are reduced compared to slits of $0.8^{\prime \prime}$ that would have been acceptable based on the seeing of the actual observations for this thesis.

\subsection{Object Selection}

A remote galaxy field will doubtless be contaminated with foreground stars and background galaxies and thus not every object observed about a remote galaxy will be a globular cluster. To maximize the ratio of detected clusters versus acquired spectra we first analyzed direct images of the galaxy field. Then, based on the expected brightness and radial distribution of the GCS of NGC 3115 , a list of likely globular clusters was created.

From the list of cluster candidates we then selected objects so as to maximize the number of objects per mask. The placement of object slits is restricted by the desired dispersion range, see Figure 7.1. For these observations the LDSS high dispersion grism was used. The dispersion is approximately $2.5 \dot{A} / C C D$ pixel. Thus to achieve a spectrum that ranges from $3500-5000 \dot{A}$ 
requires $\sim 600$ pixels. By design, the grism in the telescope is such that the middle of the spectral range $(4250 \dot{A})$ falls approximately on the same pixel that the direct image would fall on if the dispersing element was removed. Thus, to acquire a full spectrum, the object of interest must be no less then 300 pixels from the edge of the CCD detector. As a result of vignetting around the edge of the detector this restriction is somewhat larger, $\approx 400$ pixels. In fact a complete spectrum is not needed as only the feature used in the crosscorrelation needs to be imaged onto the CCD, so objects can actually be closer to the edge of the CCD ( $\sim 200$ pixels) and a velocity will still be measurable. The spectral range restriction only applies to the dispersing direction while the image-vignetting problem affects both axes.

\subsection{Mask Layout}

In order for the light to be detected on the CCD a slit or aperture must be cut into the spectrograph mask at the position of the object of interest. The size of this slit is restricted by the following considerations (see also Figure 7.2)

- The slit must be wide enough to allow most of the object light to pass through (compare object $A$ with object $C$ in Figure 7.2).

- The slit must be narrow enough so as to restrict the convolution of the point spread function of the object's image on the grism with the grism resolution.

- The slit must be narrow enough so as to prevent the stellar image from being resolved within the slit. (see Figure 7.2 object $B$ ) 
- The slit must be long enough to allow an adequate sampling of background sky light around each object.

- The slit must be short enough to allow many slits to be placed on the same mask.

From our list of target objects, seven masks which optimized the number of spectra and allowed for some duplication between masks (important for later calibration) were designed. From these seven mask layouts, three were chosen for observing. The observations for this thesis were carried out as part of a larger observing program and it was impossible to allocate a sufficient resource of time for all 7 masks to be observed. The slit size was chosen to be 1.5" in width to ensure that seeing did not restrict the level of illumination of the slit but still provided sufficient velocity resolution, and the length of the slits was allowed to vary from $10^{\prime \prime}$ to whatever size would not interfere with the spectra of neighboring objects.

[p] 


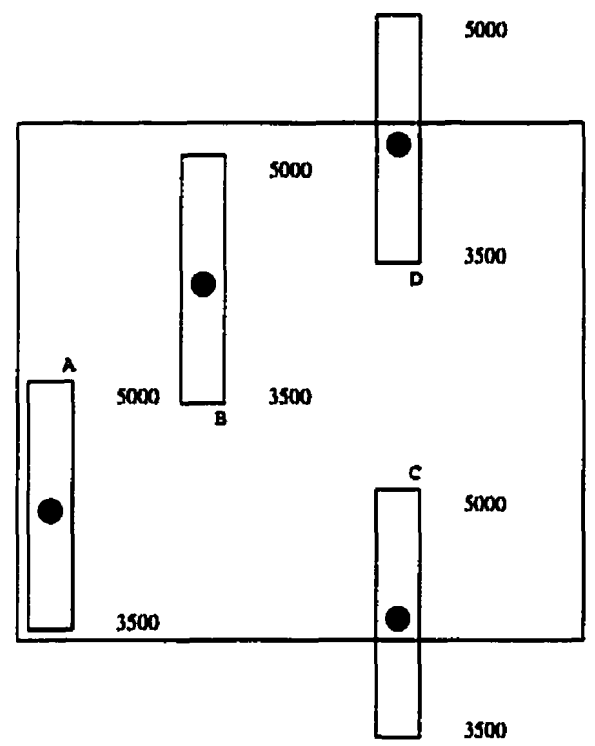

Figure 7.1 Restrictions on slit placements caused by dispersion requirements.

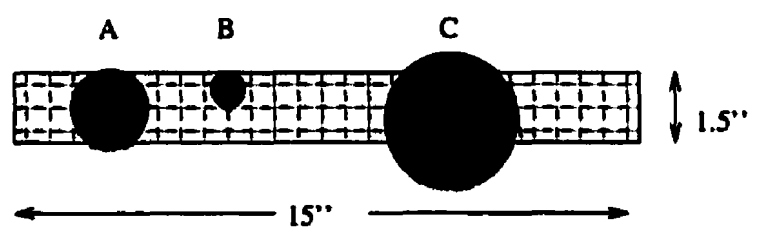

Figure 7.2 Example of mask slit sizes demonstrating image quality induced restrictions. The slit is well sampled by the CCD array (hashed background); object A makes efficient use of the slit; object B's spectrum will have a systematic offset relative to the calibrated wavelength; object $\mathrm{C}$ overfills the slit (ie not all photons from the object are allowed to reach the detector) but velocity resolution is preserved. The ideal goal is $A$ but $C$ is typical as the results of $B$ can be difficult to discover. 
TABLE 7.1. Observing Log

\begin{tabular}{|c|c|c|c|c|c|}
\hline UT & ID & $\mathrm{RA}$ & DEC & Mask & Exposure s \\
\hline 97-APR-11/12 & IQ: $1.4 "$ & & & & \\
\hline $21: 03$ & NGC 3115 & $10: 05: 13.90$ & $-07: 43: 08.5$ & $\mathrm{~m} 1$ & $2 \times 1800$ \\
\hline $22: 07$ & Cu-Ar ARC & $10: 05: 13.90$ & $-07: 43: 08.5$ & $\mathrm{~m} 1$ & $1 \times 50$ \\
\hline $22: 16$ & NGC 3115 & $10: 05: 13.90$ & $-07: 43: 08.5$ & m1 & $3 \times 1800$ \\
\hline $05: 30$ & M 13 & $16: 41: 39.20$ & $+36: 27: 31.3$ & LS & $1 \times 300$ \\
\hline $05: 40$ & M 92 & $12: 17: 07.19$ & $+43: 08: 11.3$ & LS & $1 \times 300$ \\
\hline $05: 49$ & NGC 6356 & $17: 23: 34.08$ & $-17: 48: 58.4$ LS & $1 \times 300$ & \\
\hline $06: 19$ & HD 171232 & $18: 32: 37.44$ & $+25: 29: 22.7$ & LS & $1 \times 10$ \\
\hline $06: 25$ & Cu-Ar ARC & $18: 32: 37.44$ & $+25: 29: 22.7$ & LS & $1 \times 10$ \\
\hline 97-APR-12/13 & IQ: $1.1^{\prime \prime}$ & & & & \\
\hline $20: 35$ & $\mathrm{Cu}-\mathrm{Ar}$ Arc & 08:02:13.12 & $+26: 38: 10.1$ & LS & $1 \times 30$ \\
\hline $20: 45$ & HD 65934 & $08: 02: 13.12$ & $+26: 38: 10.1$ & $\mathrm{LS}$ & $1 \times 20$ \\
\hline $20: 48$ & HD 65934 & $08: 02: 13.12$ & $+26: 38: 10.1$ & LS & $1 \times 20$ \\
\hline $21: 10$ & NGC 3115 & $10: 05: 13.90$ & $-07: 43: 08.5$ & $\mathrm{~m} 2$ & $2 \times 1800$ \\
\hline $22: 15$ & Cu-Ar Arc & $10: 05: 13.90$ & $-07: 43: 08.5$ & $\mathrm{~m} 2$ & $1 \times 50$ \\
\hline $22: 16$ & NGC 3115 & $10: 05: 13.90$ & $-07: 43: 08.5$ & $\mathrm{~m} 2$ & $2 \times 1800$ \\
\hline $04: 38$ & NGC 5024 & $13: 12: 55.22$ & $+18: 10: 08.4$ & LS & $1 \times 300$ \\
\hline $04: 49$ & NGC 6171 & $16: 32: 30.81$ & $-13: 03: 22.0$ & LS & $1 \times 300$ \\
\hline $04: 57$ & NGC 6218 & $16: 47: 14.03$ & $-01: 56: 52.0$ & LS & $1 \times 300$ \\
\hline 05:05 & NGC 5904 & $15: 18: 33.10$ & $+02: 04: 58.1$ & LS & $1 \times 300$ \\
\hline $05: 15$ & NGC 6712 & $18: 53: 02.63$ & $-08: 42: 21.0$ & $\mathrm{LS}$ & $1 \times 300$ \\
\hline $05: 24$ & NGC 7078 & $21: 29: 58.28$ & $+12: 10: 00.9$ & LS & $1 \times 300$ \\
\hline $05: 40$ & HD 132737 & $14: 59: 53.81$ & $+27: 09: 28.8$ & LS & $1 \times 50$ \\
\hline $05: 43$ & Cu-Ar Arc & $14: 59: 53.81$ & $+27: 09: 28.8$ & LS & $1 \times 50$ \\
\hline $05: 51$ & HD 194071 & $20: 22: 39.17$ & $+27: 12: 04.0$ & LS & $1 \times 50$ \\
\hline $05: 57$ & $\mathrm{Cu}-\mathrm{Ar}$ Arc & $20: 22: 39.17$ & $+27: 12: 04.0$ & LS & $1 \times 50$ \\
\hline 97-APR-13/14 & IQ: 1.6 & & & & \\
\hline $21: 20$ & NGC 3115 & $10: 05: 13.90$ & $-07: 43: 08.5$ & m5 & $3 \times 1800$ \\
\hline $22: 58$ & Cu-Ar Arc & $10: 05: 13.90$ & $-07: 43: 08.5$ & $\mathrm{~m} 5$ & $1 \times 50$ \\
\hline 23.01 & NGC 3115 & $10: 05: 13.90$ & $-07: 43: 08.5$ & m5 & $3 \times 1800$ \\
\hline $05: 34$ & hd 132737 & $14: 59: 53.81$ & $+27: 09: 28.8$ & LS & $1 \times 30$ \\
\hline $05: 42$ & hd 171232 & $18: 32: 37.30$ & $+25: 29: 21.5$ & LS & $1 \times 30$ \\
\hline $05: 50$ & hd 171232 & $18: 32: 37.30$ & $+25: 29: 21.5$ & LS & $1 \times 15$ \\
\hline $05: 54$ & hd 194071 & $20: 22: 39.23$ & $+28: 14: 49.6$ & LS & $1 \times 20$ \\
\hline
\end{tabular}




\subsubsection{Preprocessing}

First the bias level was removed as described in section 5.3.1. Then, for spectroscopic CCD data the preprocessing required depends on the end use of the data. If the absolute signal strength is important, as for line-strength analysis, then knowledge of the CCD+telescope optics response is needed as well as information regarding the illumination of the slit (the slit function). If, however, the extracted spectra will not be flux calibrated then absolute measure of the response is not required and only the small scale (pixel-pixel) variations need to be removed. The use of absolute calibration was not required for this project as the objects being examined are very faint and the amount of exposure time available was too limited to allow line strength measurements to be made.

To remove the small scale detector sensitivity variations from the data requires the even illumination of the slit mask. In addition the illumination source must be free of emission lines and absorption features. To achieve this goal the telescope is illuminated via a quartz lamp which contains no spectral emission lines or absorption features and only a broad/smooth response function. For each row of pixels in the dispersion direction a high order (50) cubic spline is fit to the spectral shape of the quartz lamp and each pixel value is divided by its fit value. The resulting form then provides a normalized dataset which only varies due to changes in local pixel sensitivity. This procedure leaves the broad-scale features, caused by vignetting of the CCD, but the broad-scale features are of no relevance as each spectrum will be individually continuum subtracted prior to the cross-correlation procedure.

With the individual frames flat fielded and bias corrected, the data can now be processed to extract the astrophysically significant information. 


\subsection{Spectral Extraction}

\subsubsection{The Program Frames}

Each of the program masks was imaged multiple times, see the bold entries in Table 7.1. Between these exposures the telescope was repositioned (tweaked) to optimize the placement of the target objects in the mask slits. As the actual mask did not move relative to the grism there should be no variation in the wavelength calibration between tweaked images. To verify that this is the case the spectrum of one object was extracted from each of the exposures separately. These spectra were then cross-correlated on a pixel scale with no measurable shift in the spectra detected, i.e., the wavelength scale of the slit did not change from exposure to exposure and no offset of images was required before the frames could be combined to form a master image.

Given that there was no offset between exposures of the same mask, the exposures for each mask were combined. Pixels which deviated from the average by more than an amount that would be consistent with the noise level were flagged as deviant and the average was then performed without the deviant pixels. This rejection procedure (crreject in IRAFs imcombine task) ensures that a majority of the cosmic ray strikes are removed from the images.

Using the IRAF package rgo.sam, each program image was examined and the slit spectra were extracted. To extract the spectra a window of the digitized CCD image is defined which contains the entire spectral run of the slit of interest and extends spatially to the edges of the slit. Then, using a plot across the spatial extent of the slit, a region which contains the stellar signal and two regions which define a sky background are selected. The signal across the spatial extent of the slit is then combined for each pixel row (ie each 
spectral resolution element). An optimal weighting scheme ensures that the pixels which contain the majority of the light from the object of interest are weighted more heavily than pixels from the wings of the light profile (Horne 1986). During the extraction, the sky across the spatial extent of the slit is modeled, using a third order polynomial fit to the pixels that were selected as the sky background.

Once the extraction region was defined for the program frame, the same region was extracted from the calibration arc exposure. The extraction of arc lines does not require weighting and no background subtraction is performed. The arc spectra were then examined and the wavelength/pixel calibration was defined using the imred.specred.identify task in IRAF and a dispersion solution was found. The dispersion solution was fit using a first order cubic spline resulting in typical RMS residuals of $0.08 \dot{A}$. That is to say, that arc features were found (on average) within $0.08 \dot{\mathrm{A}}$ of their true values, this translates into a velocity uncertainty of $\sim 6 \mathrm{~km} / \mathrm{s}$ at $4000 \dot{\mathrm{A}}$ resulting from the wavelength calibration.

\subsubsection{Radial Velocity Standards}

The object of the observations was to acquire the spectra of targets around NGC 3115 that were in the right brightness range to be considered globular clusters. Determining the radial velocities of these objects requires crosscorrelating their spectra with the spectra of templates of similar composition and the template spectra should be of high quality. Thus we chose to use the smeared light from metal-poor galactic globulars as our template objects as this would simulate the expected spectrum of the metal-poor halo clusters of NGC 3115. The spectra were acquired by placing a long slit in the light 
path and tracking the cluster at a non-sidereal rate, thus smearing the cluster image across the slit. These template object spectra were then extracted and calibrated in a fashion similar to that described for the program frames.

In addition to the cluster templates, radial velocity standard stars were also observed. This was required as some of the objects acquired as cluster candidates would, inevitably, turn out to be field stars and as such would correlate with stellar spectra better than the integrated spectra of old halo clusters. This also allows us the opportunity to verify the velocity scale of our template set of MW clusters. The standard templates used are summarized in Table 7.1, the templates are the targets observed using the long slit (LS) mask.

\subsection{Velocity Calibration}

Unlike the program frames the observations of arc spectra did not always occur before the long-slit mask had been moved. Thus there is a possibility that the long-slit mask arcs do not exactly register with the long-slit spectra we acquired. Table 7.1 indicates the arc used for each of template observations. In order to test the velocity calibration the radial velocity of each template was determined by measuring the central wavelength of the Fraunhofer $\mathrm{H} \& \mathrm{~K}$ absorption features, see Figure 7.3, and comparing the measured wavelength with the rest frame value, see Table 7.2. The velocities for each of the templates were then compared with the catalogued velocities, see Table 7.3. From this comparison an obvious systematic difference between measured and catalogued velocities were found. From Figure 7.4 there is clearly a trend of offset in the measured velocity as a function of the hour angle of the observation for the globular cluster templates and a systematic zero point shift for the velocity 
standard stars.

The shift of the cluster velocities is likely due to flexure that occurs in the telescope as it slews to large hour angles. As no spectral arcs were taken for the globular cluster velocity standards at large hour angles, the flexure of the telescope manifests itself as a trend of velocity offset with hour angle. The two clusters observed at large negative hour angles will not be used for velocity measurements, owing to the large flexure in the spectrograph at these large hour angles. The mean offset between the measured and catalogued velocity for the clusters observed at positive hour angle was approximately 50 pixels. So a shift of 0.5 pixels was applied to the cluster spectra to remove this offset.

The radial velocity standards are free of flexure problems as arcs were always acquired immediately before or after the spectrum of the standard with the telescope orientation remaining fixed. However, as the seeing profiles of the radial velocity standard stars were resolved in the slit (owing to the good seeing conditions) there is a systematic velocity shift for these stars. The velocity shift of the standard stars are approximately equal as each stellar profile was aligned to the same pixel in the long slit mask, ie offset to the same position within the slit. To correct for this misalignment the spectra of the HD stars were shifted by 0.75 pixels.

As these two shifts have been applied independently they can now be checked by comparing the velocity that each type of template gives for a program object. If the shifts were incorrectly applied then the velocity as determined using HD stars should differ from that found using the cluster templates. To this end three program objects were cross-correlated with each of the templates and the resulting velocities were examined for any systematic difference between the values found using the HD stars and those found using 
TABLE 7.1. (continued)

\begin{tabular}{cccccc}
\hline UT & ID & RA & DEC & Mask & Exposure s \\
\hline $05: 54$ & hd 194071 & 20:22:39.23 & $+28: 14: 49.6$ & LS & $1 \times 5$ \\
\hline \hline
\end{tabular}

Notes to Table 7.1.

Observations taken at the WHT using the LDSS. Program frames appear in bold. Various detector calibration frames (flat, biases and darks) are not included. Exposure times are in seconds. $\mathrm{LS}==$ long slit mask, $\mathrm{ml}==$ mask $1, \mathrm{~m} 2==$ mask $2, \mathrm{~m} 5==$ mask 5 .

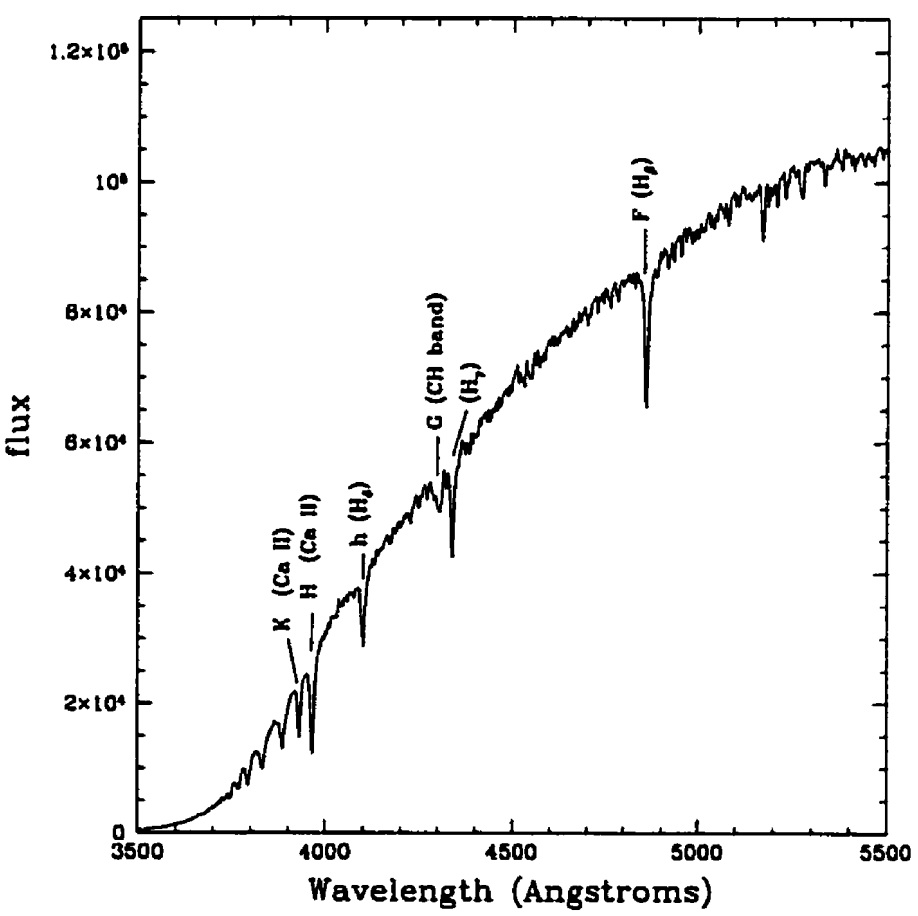

Figure 7.3 Spectrum of M92 showing the major features. Note that the shape of the continuum has not been corrected for the response of the CCD and optics. Features are labeled with their designations by Fraunhofer and the atomic element is shown in braces 
TABLE 7.2. Fraunhofer lines.

\begin{tabular}{|c|c|}
\hline Wavelength & Designation \\
\hline 7594 & $\mathrm{~A}$ (terrestrial $\left.\mathrm{O}_{2}\right)$ \\
\hline 7165 & a (water vapour) \\
\hline 6867 & $\mathrm{~B}\left(\right.$ terrestrial $\left.\mathrm{O}_{2}\right)$ \\
\hline 6563 & $\mathrm{C}\left(\mathrm{H}_{\alpha}\right)$ \\
\hline 5890 & D1 (Na doublet) \\
\hline 5896 & D2 \\
\hline 5270 & $\mathrm{E} 1(\mathrm{Fe})$ \\
\hline 5167 & $\mathrm{E}_{b}$ (MgI triplet) \\
\hline \multicolumn{2}{|l|}{5173} \\
\hline \multicolumn{2}{|l|}{5184} \\
\hline 4861 & $\mathrm{~F}\left(\mathrm{H}_{\beta}\right)$ \\
\hline 4384 & $d(\mathrm{FeI})$ \\
\hline 4300 & $\mathrm{G}$ band (CH\&Fe) \\
\hline 4227 & $\mathrm{~g}(\mathrm{CaI})$ \\
\hline 4102 & $\mathrm{~h}\left(\mathrm{H}_{\delta}\right)$ \\
\hline 3968 & $\mathrm{H}(\mathrm{CaII})$ \\
\hline 3934 & $\mathrm{~K}(\mathrm{CaII})$ \\
\hline
\end{tabular}




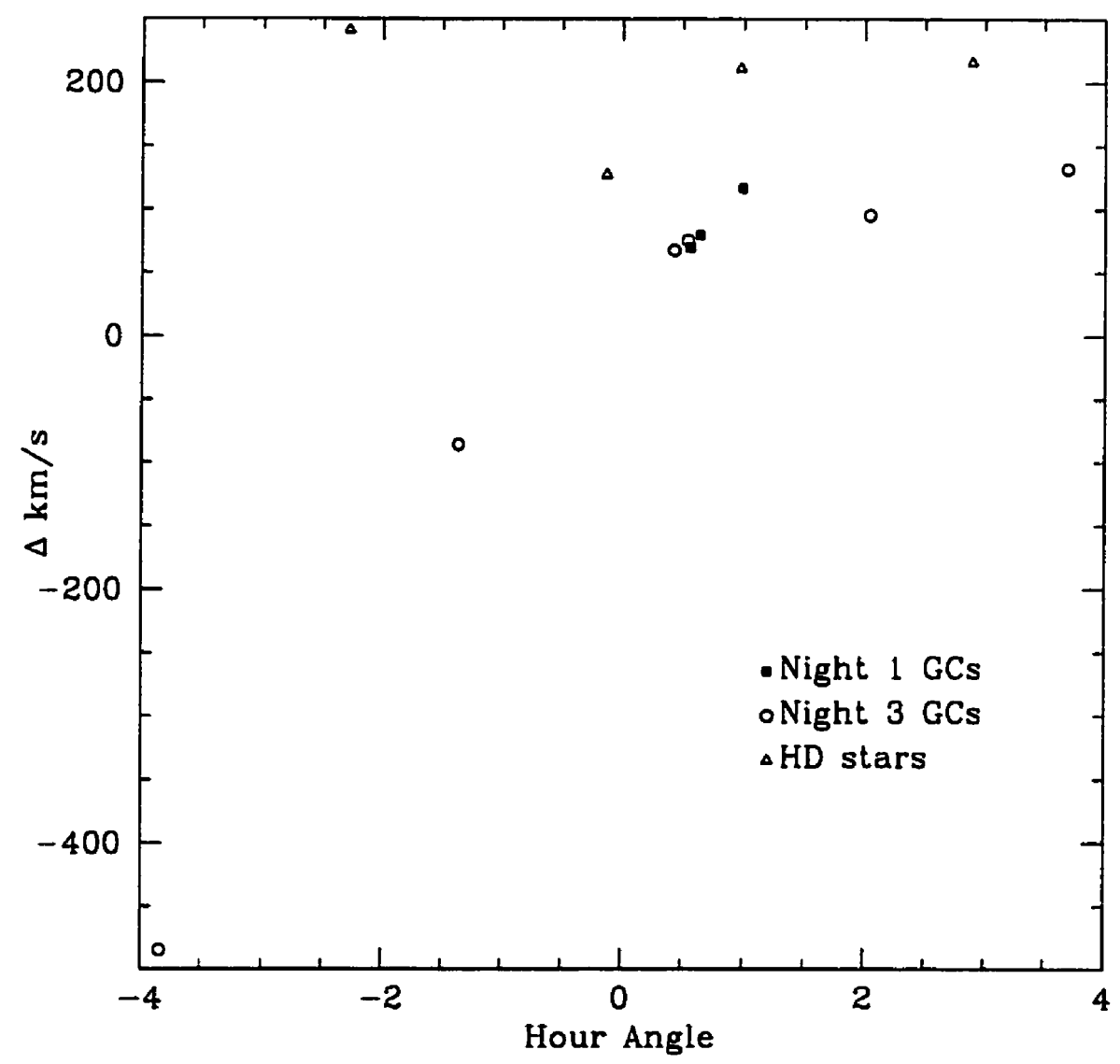

Figure 7.4 The offset in measured radial velocity from that expected for the radial velocity standards. The globular clusters at large negative hour angle were rejected (see text) and the remaining standards were shifted to zero residual velocity (see text). 
TABLE 7.3. Radial Velocity Standards

\begin{tabular}{clll}
\hline \hline Object & $v_{\text {Helio }}$ & $\Delta v$ & Hour Angle \\
\hline NGC 5024 & -79 & 132 & 3.69 \\
NGC 5904 & 51.0 & 95 & 2.04 \\
NGC 6171 & -34.8 & 75 & 0.54 \\
NGC 6205 & -24.6 & 116 & 0.99 \\
NGC 6218 & -43.5 & 67 & 0.43 \\
NGC 6341 & -120 & 69 & 0.56 \\
NGC 6356 & 25.6 & 79 & 0.64 \\
NGC 6712 & -107 & -86 & -1.36 \\
NGC 7078 & -106 & -485 & -3.84 \\
HD 132737 & -24.1 & 216 & 2.89 \\
HD 171232 & -35.90 & 127 & -0.13 \\
HD 194071 & -9.80 & 241 & -2.29 \\
HD 65934 & 35.00 & 211 & 0.97 \\
\hline \hline
\end{tabular}

Notes to Table 7.3.

The catalogue values for the radial velocity standards along with the difference between the catalogue value and that measured using the $\mathrm{H} \& \mathrm{~K}$ Fraunhofer lines, in the sense $v_{\text {Helio }}-v_{\text {Fraunhofer }}$. 
the cluster templates. No difference was found and so the zero-points of the two templates sets are consistent. The program spectra are free of both these concerns as a) arc spectra were acquired without variation of the telescope (no flexure change) and b) the multiple slits of the program field were aligned using multiple guide holes, ensuring that each object fell in the middle of its mask slit.

\subsection{Velocities}

\subsubsection{Cross Correlation}

After extracting the spectra from the program frames they were cross-correlated against each of the velocity templates using the rv.fxcor task in IRAF. The procedure followed was to first divide out the continuum, modelled by a cubic spline fit to a smoothed version of the spectrum. Then the continuum corrected spectra was re-binned onto a log wavelength scale and cross-correlated with a similarly prepared template, using a Fourier cross-correlation (see Tonry and Davis 1979). The central peak position of the cross-correlation spectra was determined by fitting a Gaussian to the peak. For a cross-correlation to be considered valid required that the cross-correlation coefficient, $C C$, (the height of best fit Gaussian) exceeds 0.30 for an individual template or that the velocities returned using multiple templates, each with $C C \gtrless 0.25$, agreed to within the uncertainty of the $C C$ peak. This restriction on the $C C$ peak ensures that the velocity returned by the $C C$ has a high likelihood of being meaningful (Tonry and Davis 1979). The resulting velocities are listed in Table 7.5. 


\subsubsection{Internal Dispersion}

There are 5 objects in common between mask 1 and 5 , and 3 objects in common between mask 2 and 5 . In cases where a valid cross correlation was possible in both masks the difference between the two velocities gives some indication of the internal velocity dispersion. However, only a few pairs had velocities, as some of the common objects had different spectral features from those of the templates. In these cases the two program spectra were cross-correlated to provide a velocity difference between the two masks being compared. The results are listed in Table 7.4. No systematic difference between the masks was found. The uncertainty in a single velocity determination was found to be $\sim 90 \mathrm{~km} / \mathrm{s}$. 
TABLE 7.4. Velocity scatter.

\begin{tabular}{ll}
\hline Object & \multicolumn{1}{c}{$\Delta(\mathrm{km} / \mathrm{s})$} \\
\hline & mask-2 - mask-5 \\
184 & 43 \\
245 & 10 \\
263 & -82 \\
mean & $-9 \pm 65$ \\
& \\
69 & mask-1 - mask-5 \\
77 & -113 \\
98 & 179 \\
404 & -145 \\
490 & 88 \\
mean & 139.0 \\
\hline \hline
\end{tabular}

\section{Notes to Table 7.4.}

Difference in velocity for the same object measured in different masks. There is no statistically significant difference between the velocities as measured in mask-1 versus 5 or mask-2 versus 5 . There were no mask-2 : mask-1 pairs. 
TABLE 7.5. Cross correlation velocities.

\begin{tabular}{|c|c|c|c|c|}
\hline Slit & RA & DEC & $v_{\text {helio }} \mathrm{km} / \mathrm{s}$ & $\Delta v \mathrm{~km} / \mathrm{s}$ \\
\hline 9006 & $10: 04: 53.8$ & $-7: 40: 15$ & $\ldots$ & $\cdots$ \\
\hline 306 & $10: 04: 54.0$ & $-7: 38: 58$ & $\cdots$ & $\cdots$ \\
\hline 306 & $10: 04: 54.0$ & $-7: 38: 58$ & $\cdots$ & $\cdots$ \\
\hline 319 & $10: 04: 55.1$ & $-7: 48: 06$ & $\cdots$ & $\cdots$ \\
\hline 499 & $10: 04: 55.2$ & $-7: 43: 38$ & $\cdots$ & $\cdots$ \\
\hline 269 & $10: 04: 55.3$ & $-7: 44: 54$ & $\cdots$ & $\cdots$ \\
\hline 9061 & $10: 04: 55.6$ & $-7: 42: 10$ & -44 & 16 \\
\hline 320 & $10: 04: 55.7$ & $-7: 43: 51$ & 520 & 91 \\
\hline 283 & $10: 04: 56.8$ & $-7: 47: 42$ & $\cdots$ & $\ldots$ \\
\hline 283 & $10: 04: 56.8$ & $-7: 47: 42$ & $\cdots$ & $\cdots$ \\
\hline 490 & $10: 04: 57.5$ & $-7: 39: 20$ & $\cdots$ & $\cdots$ \\
\hline 367 & $10: 04: 57.6$ & $-7: 42: 56$ & -76 & 3 \\
\hline 536 & $10: 04: 57.6$ & $-7: 40: 07$ & $\cdots$ & $\cdots$ \\
\hline 521 & $10: 04: 58.2$ & $-7: 45: 30$ & $\cdots$ & $\ldots$ \\
\hline 521 & $10: 04: 58.2$ & $-7: 45: 30$ & $\cdots$ & $\cdots$ \\
\hline 85 & $10: 04: 58.2$ & $-7: 43: 31$ & $\ldots$ & $\cdots$ \\
\hline 435 & $10: 04: 58.3$ & $-7: 40: 42$ & -65 & 3 \\
\hline 9136 & $10: 04: 58.3$ & $-7: 46: 31$ & 695 & 15 \\
\hline 183 & 10:04:59.1 & $-7: 46: 58$ & 47385 & 3 \\
\hline 9023 & 10:04:59.1 & $-7: 46: 58$ & 31640 & 3 \\
\hline 190 & $10: 04: 59.2$ & $-7: 47: 32$ & $\ldots$ & $\ldots$ \\
\hline 256 & $10: 04: 59.2$ & $-7: 42: 15$ & $\cdots$ & $\ldots$ \\
\hline 97 & $10: 04: 59.3$ & $-7: 46: 11$ & 409 & 3 \\
\hline 69 & $10: 04: 59.7$ & $-7: 39: 49$ & ... & $\cdots$ \\
\hline 95 & $10: 04: 60.0$ & $-7: 45: 35$ & ... & ... \\
\hline 154 & $10: 05: 00.6$ & $-7: 41: 19$ & 13 & 6 \\
\hline 83 & 10:05:03.0 & $-7: 42: 34$ & ... & $\ldots$ \\
\hline 140 & $10: 05: 03.1$ & $-7: 39: 34$ & -54 & 34 \\
\hline 312 & $10: 05: 03.8$ & $-7: 40: 27$ & $\ldots$ & ... \\
\hline 523 & $10: 05: 04.2$ & $-7: 38: 55$ & 69 & 15 \\
\hline 578 & $10: 05: 04.3$ & $-7: 40: 18$ & $\ldots$ & $\ldots$ \\
\hline 175 & $10: 05: 04.5$ & $-7: 45: 45$ & $\ldots$ & ... \\
\hline 9004 & $10: 05: 04.6$ & $-7: 45: 45$ & $\cdots$ & $\cdots$ \\
\hline 242 & $10: 05: 04.8$ & $-7: 40: 04$ & $\ldots$ & $\ldots$ \\
\hline
\end{tabular}


TABLE 7.5. (continued)

\begin{tabular}{|c|c|c|c|c|}
\hline Slit & $\mathrm{RA}$ & DEC & $v_{\text {helio }} \mathrm{km} / \mathrm{s}$ & $\Delta v \mathrm{~km} / \mathrm{s}$ \\
\hline 9089 & $10: 05: 05.6$ & $-7: 48: 06$ & $\ldots$ & $\ldots$ \\
\hline 9077 & $10: 05: 05.7$ & $-7: 41: 42$ & 833 & 17 \\
\hline 430 & $10: 05: 07.4$ & $-7: 38: 40$ & $\ldots$ & $\cdots$ \\
\hline 195 & $10: 05: 07.8$ & $-7: 47: 54$ & $\cdots$ & $\cdots$ \\
\hline 569 & $10: 05: 07.8$ & $-7: 44: 34$ & $\cdots$ & $\cdots$ \\
\hline 144 & $10: 05: 07.9$ & $-7: 39: 56$ & 573 & 20 \\
\hline 195 & $10: 05: 07.9$ & $-7: 47: 53$ & $\cdots$ & $\cdots$ \\
\hline 561 & 10:05:09.1 & $-7: 44: 38$ & 650 & 7 \\
\hline 132 & $10: 05: 09.2$ & $-7: 38: 48$ & $\ldots$ & $\cdots$ \\
\hline 363 & 10:05:09.5 & $-7: 42: 01$ & 614 & 17 \\
\hline 129 & $10: 05: 09.7$ & $-7: 38: 33$ & 563 & 18 \\
\hline 55 & $10: 05: 09.7$ & $-7: 48: 04$ & $\ldots$ & $\ldots$ \\
\hline 173 & $10: 05: 09.9$ & $-7: 45: 00$ & -129 & 7 \\
\hline 108 & $10: 05: 11.3$ & $-7: 48: 07$ & $\ldots$ & $\ldots$ \\
\hline 9038 & $10: 05: 11.3$ & $-7: 42: 22$ & $\ldots$ & $\ldots$ \\
\hline 9008 & $10: 05: 11.4$ & $-7: 45: 00$ & 385 & 4 \\
\hline 9053 & $10: 05: 12.5$ & $-7: 44: 07$ & 322 & 16 \\
\hline 588 & $10: 05: 12.7$ & $-7: 42: 43$ & $\ldots$ & $\cdots$ \\
\hline 527 & $10: 05: 13.1$ & $-7: 43: 58$ & 421 & 13 \\
\hline 566 & $10: 05: 13.1$ & $-7: 42: 18$ & 794 & 35 \\
\hline 583 & $10: 05: 14.9$ & $-7: 41: 51$ & 52450 & 3 \\
\hline 498 & $10: 05: 15.8$ & $-7: 43: 17$ & $\cdots$ & $\cdots$ \\
\hline 333 & $10: 05: 16.0$ & $-7: 47: 00$ & -62 & 32 \\
\hline 238 & $10: 05: 16.6$ & $-7: 39: 33$ & $\ldots$ & $\ldots$ \\
\hline 181 & $10: 05: 16.8$ & $-7: 46: 47$ & 469 & 16 \\
\hline 98 & $10: 05: 16.8$ & $-7: 46: 12$ & 47340 & 28 \\
\hline 192 & $10: 05: 17.1$ & $-7: 47: 37$ & $\ldots$ & $\ldots$ \\
\hline 538 & $10: 05: 17.1$ & $-7: 42: 47$ & 630 & 15 \\
\hline 567 & $10: 05: 17.1$ & $-7: 43: 55$ & 72753 & 3 \\
\hline 9002 & $10: 05: 17.1$ & $-7: 43: 55$ & 72814 & 3 \\
\hline 9019 & $10: 05: 17.1$ & $-7: 47: 36$ & $\cdots$ & $\cdots$ \\
\hline 9055 & $10: 05: 17.1$ & $-7: 43: 20$ & $\cdots$ & $\cdots$ \\
\hline 497 & $10: 05: 18.2$ & $-7: 42: 58$ & $\ldots$ & $\cdots$ \\
\hline 330 & $10: 05: 19.1$ & $-7: 46: 24$ & 47462 & 3 \\
\hline
\end{tabular}


TABLE 7.5. (continued)

\begin{tabular}{|c|c|c|c|c|}
\hline Slit & RA & DEC & $v_{\text {helio }} \mathrm{km} / \mathrm{s}$ & $\Delta v \mathrm{~km} / \mathrm{s}$ \\
\hline 9065 & $10: 05: 19.3$ & $-7: 41: 56$ & $\cdots$ & $\cdots$ \\
\hline 253 & $10: 05: 20.1$ & $-7: 41: 44$ & 615 & 6 \\
\hline 245 & $10: 05: 20.3$ & $-7: 40: 09$ & 268 & 5 \\
\hline 252 & $10: 05: 21.1$ & $-7: 41: 37$ & 893 & 7 \\
\hline 9073 & $10: 05: 21.2$ & $-7: 46: 45$ & $\ldots$ & $\ldots$ \\
\hline 531 & $10: 05: 22.7$ & $-7: 45: 27$ & 107 & 34 \\
\hline 313 & $10: 05: 22.8$ & $-7: 41: 06$ & 651 & 115 \\
\hline 404 & $10: 05: 22.9$ & $-7: 38: 23$ & $\cdots$ & $\ldots$ \\
\hline 263 & 10:05:23.1 & $-7: 43: 32$ & 250 & 20 \\
\hline 472 & $10: 05: 23.4$ & $-7: 44: 07$ & 31 & 15 \\
\hline 368 & $10: 05: 23.8$ & $-7: 43: 29$ & -0.23 & 24 \\
\hline 184 & $10: 05: 23.9$ & $-7: 47: 00$ & 44123 & 3 \\
\hline 331 & $10: 05: 23.9$ & $-7: 46: 26$ & $\cdots$ & $\cdots$ \\
\hline 9092 & $10: 05: 24.2$ & $-7: 39: 09$ & 780 & 45 \\
\hline 431 & $10: 05: 25.0$ & $-7: 39: 00$ & 275 & 33 \\
\hline 326 & $10: 05: 25.2$ & $-7: 45: 00$ & 74 & 6 \\
\hline 9015 & 10:05:25.2 & $-7: 45: 21$ & $\cdots$ & $\cdots$ \\
\hline 530 & $10: 05: 25.3$ & $-7: 45: 16$ & -45 & 15 \\
\hline 308 & $10: 05: 25.8$ & $-7: 39: 49$ & $\cdots$ & $\cdots$ \\
\hline 327 & 10:05:26.1 & $-7: 45: 32$ & 959 & 8 \\
\hline 9109 & $10: 05: 26.2$ & $-7: 41: 44$ & $\cdots$ & $\cdots$ \\
\hline 9022 & $10: 05: 26.8$ & $-7: 39: 25$ & $\cdots$ & $\cdots$ \\
\hline 137 & $10: 05: 26.9$ & $-7: 39: 24$ & $\cdots$ & $\cdots$ \\
\hline 153 & $10: 05: 27.9$ & $-7: 41: 14$ & $\cdots$ & $\cdots$ \\
\hline 72 & $10: 05: 28.0$ & $-7: 40: 04$ & 79 & 21 \\
\hline 77 & $10: 05: 28.0$ & $-7: 40: 50$ & $\cdots$ & $\cdots$ \\
\hline 136 & $10: 05: 29.7$ & $-7: 39: 06$ & $\cdots$ & $\cdots$ \\
\hline 233 & $10: 05: 31.0$ & $-7: 38: 46$ & $\cdots$ & $\cdots$ \\
\hline 105 & $10: 05: 32.1$ & $-7: 47: 57$ & $\cdots$ & $\ldots$ \\
\hline 445 & 10:05:33.4 & $-7: 46: 10$ & $\ldots$ & $\cdots$ \\
\hline 240 & $10: 05: 33.7$ & $-7: 39: 40$ & $\cdots$ & $\cdots$ \\
\hline
\end{tabular}

Notes to Table 7.5.

The entries marked $\ldots$ would not cross-correlate with the templates. 


\section{Chapter 8}

\section{Velocity Analysis}

In this chapter I present the results of our analysis of the velocities of objects around NGC 3115. In Section 8.1 I describe the choice of velocity cut which selects the globular clusters about NGC 3115 . Section 8.2 presents evidence that the cluster system is rotating. In Section 8.3 the mass-to-light ratio of NGC 3115 is determined.

\subsection{The Velocity Cut}

The heliocentric radial velocity of NGC 3115 is $v_{\odot}=663 \pm 6 \mathrm{~km} / \mathrm{s}$ (Capaccioli, Cappellaro, Held, and Vietri 1993) and we therefore expect that objects in bound orbits (ie clusters) should have helio-centric velocities with $350<v_{\odot}<1050 \mathrm{~km} / \mathrm{s}$. The histogram of velocities in Figure 8.1 shows a suggestive break in the velocity distribution at $v_{\odot}=150 \mathrm{~km} / \mathrm{s}$ with a group of three objects in the range of $250-300 \mathrm{~km} / \mathrm{s}$. The cut at $150 \mathrm{~km} / \mathrm{s}$ is reasonable but perhaps somewhat low; in addition, this velocity cut is asymmetric about the systemic velocity of NGC 3115 as no objects were found with velocities above $\sim 1000 \mathrm{~km} / \mathrm{s}$. Figure 8.2 is a colour-magnitude diagram for objects 
with $250<v_{\odot}<1000 \mathrm{~km} / \mathrm{s}$. From this diagram we see that the objects with $v_{\odot}<300 \mathrm{~km} / \mathrm{s}$ are all very blue, accounting for three of the four objects with $V-I<0.8$ (outside the range of colour used to select clusters in section 6.2) and may in fact be old metal-poor halo stars. Thus we eliminated the three low velocity objects and made the cut at $v_{\odot}>300 \mathrm{~km} / \mathrm{s}$, which results in a distribution which is more symmetric about the systemic velocity of NGC 3115. If these three objects were included in the analysis but are in fact halo stars the result would be an over estimate of the true mass of NGC 3115. By not including these points the mass derived below, and more importantly the evidence of a dark halo, is more robust.

\subsection{Rotation}

Is the cluster system of NGC 3115 rotating? This question is key to understanding the formation of cluster systems and galaxies. Figure 8.3 presents the radial velocity as a function of the projected major-axis distance of the cluster from the center of the galaxy. Objects at positive major axis have velocities larger (on average) than the systemic velocity of the galaxy and objects at negative major axis position have velocities lower (on average) than the systemic velocity of the galaxy. A linear regression reveals a relation of

$$
v_{\odot}(A)=633+44 * A \pm 15 \mathrm{~km} / \mathrm{s}
$$

where $\mathrm{A}$ is the projected semi-major axis position of the object in arc-minutes. The linear correlation co-efficient is $R=0.76$, ie. there is only a $0.5 \%$ probability that the correlation found would occur randomly given the number of data points. The uncertainty quoted is the mean residual of the fit and does not account for possible systematic effects. The systemic velocity of the clus- 


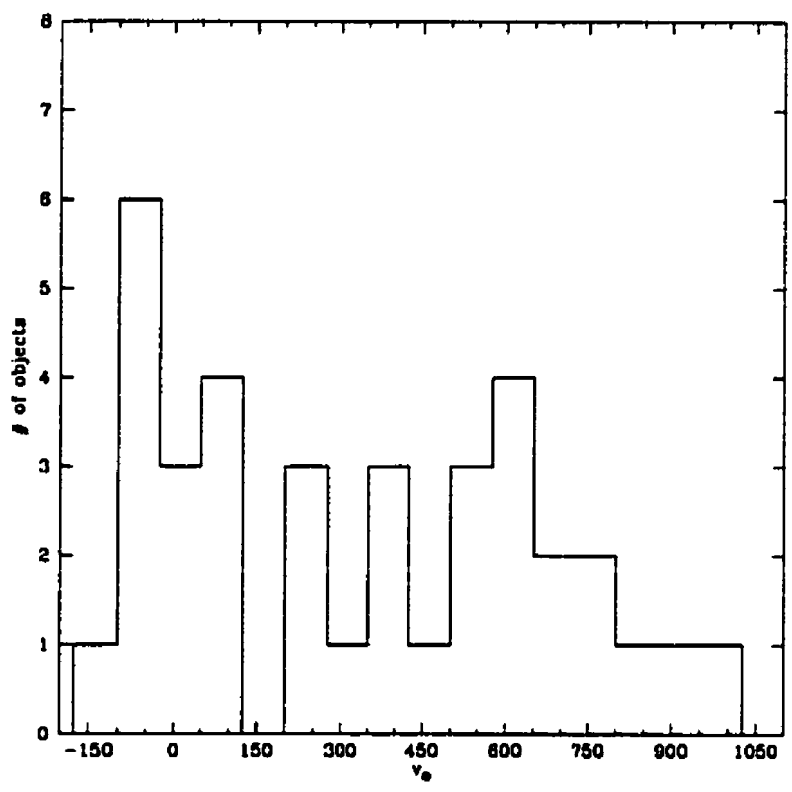

Figure 8.1 Histogram of velocities for all objects for which radial velocities could be determined (excluding those objects which are obviously galaxies). The objects clustered near $0 \mathrm{~km} / \mathrm{s}$ are halo field stars. The objects in the $225 \mathrm{~km} / \mathrm{sto} 275 \mathrm{~km} / \mathrm{s}$ bin are rejected on the basis of colour as unlikely to be globular clusters (see text). 


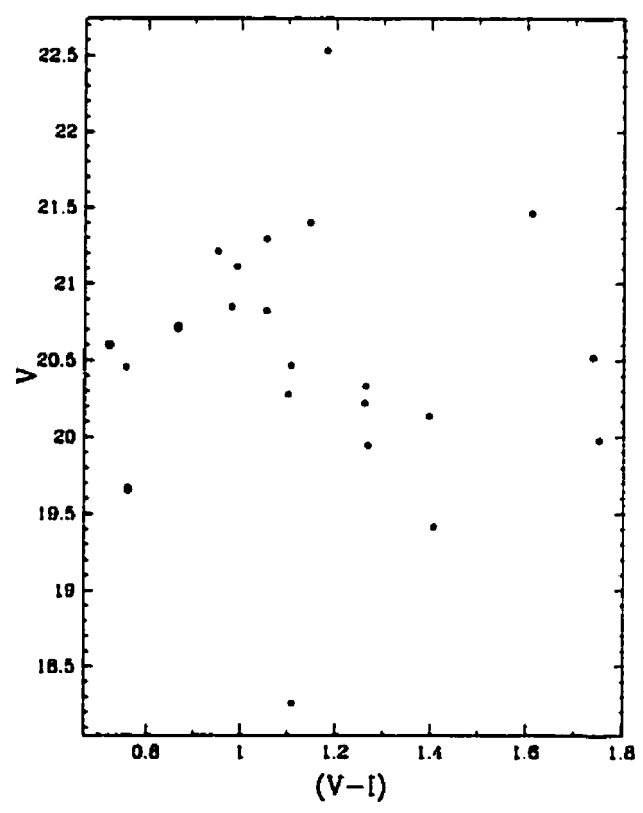

Figure 8.2 A colour magnitude diagram of objects with $250<v_{\odot}<1000 \mathrm{~km} / \mathrm{s}$. Objects with $v_{\odot}<300 \mathrm{~km} / \mathrm{s}$ are given as filled circles and objects with $v_{\odot}>$ $300 \mathrm{~km} / \mathrm{s}$ are shown as open circles. 
ter system, $633 \pm 15 \mathrm{~km} / \mathrm{s}$, is in good agreement with the systemic velocity of NGC $3115(663 \pm 6 \mathrm{~km} / \mathrm{s})$.

\subsection{The Mass of NGC 3115}

Determining the mass of an object with particles in orbit requires a knowledge of the orbits that those particles are on. Unfortunately it is impossible to determine exactly the orbits that are being followed when viewing a system in projection as we only have access to velocity and position information in the projected plane (ie only 2 constraints on the six degrees of freedom in the orbit). However, if we assume the specific distribution of orbits (which then act as a constraint of the remaining 4 degrees of freedom) it is possible to estimate the mass of the galaxy. Traditionally the viral theorem is turned to in such circumstance but the viral mass estimator is biased and not efficient, while the projected mass estimator (PME) provides a more robust, unbiased, and efficient tool (Bachall and Scott 1981).

\subsubsection{The Projected Mass Estimator}

The PME is

$$
M=f \frac{1}{N G} \sum_{i=1}^{N} v_{z i}^{2} * R_{i}
$$

where $f$ is an empirical scale factor determined by the the shape of the orbits, $M$ is the mass estimate, $N$ is the number of objects for which we have radial velocities, $v_{z i}$ is the line-of-sight velocity (after removal of the systemic velocity and any rotational component), $R_{i}$ is the projected radius of the ith object and $G$ is Newton's constant. The two extremes of orbit types, isotropic orbits and linear orbits yield 

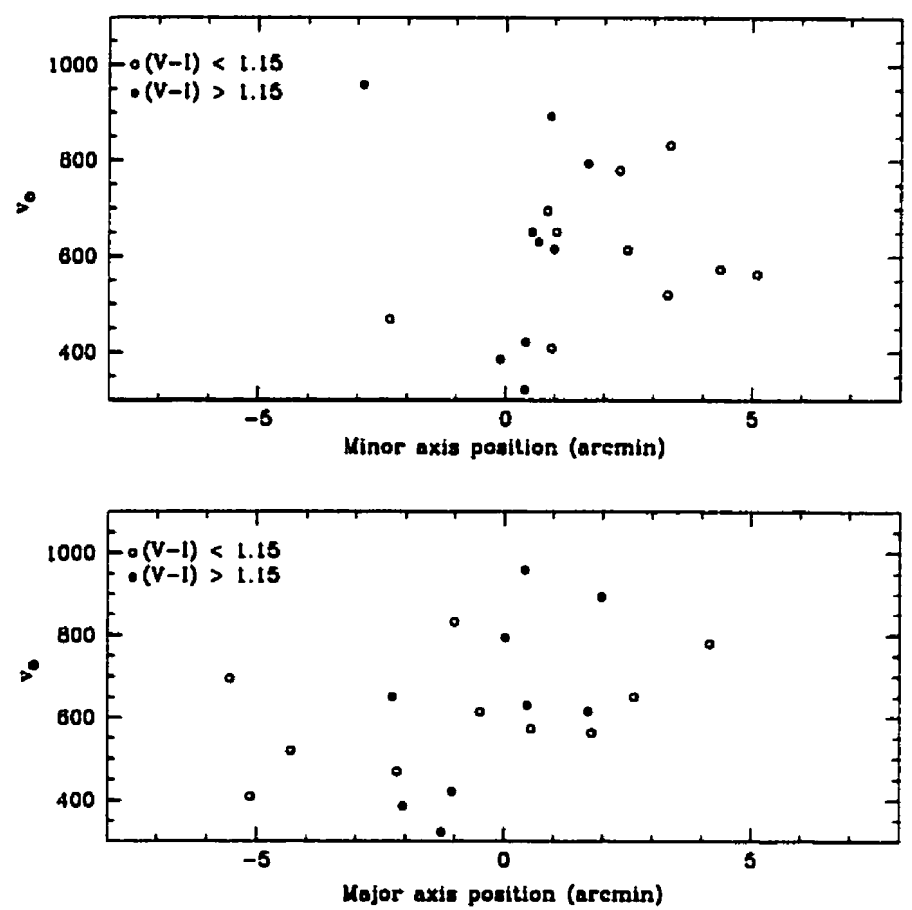

Figure 8.3 Radial velocities as a function of projected position relative to the major and minor axes of NGC 3115. Notice that the red objects exhibit a larger velocity dispersion and larger rotation velocity than do the blue objects. 


$$
\begin{array}{ll}
f=16 / \pi & \text { isotropic orbits } \\
f=32 / \pi & \text { linear orbits }
\end{array}
$$

For the case of the GCS of NGC 3115 we have already determined that there is some rotation and so the linear orbit value is an obvious overestimate, however the orbits are not likely to be purely isotropic either. We use here a value of $f=\frac{64}{3 \pi}$. If the orbits of the clusters follow exactly the shape of the light profile (an unlikely case but more realistic perhaps than linear orbits) then the normalization

$$
f=\frac{32}{\pi\left(3-2<e^{2}>\right)}
$$

where $\left\langle e^{2}\right\rangle$ is the mean eccentricity. Thus using $\langle e\rangle=0.4$ gives $f=\frac{32}{2.68 \pi}$ where the value of $\langle e\rangle=0.4$ is the eccentricity of the galaxy light profile (Bachall and Scott 1981). Thus a value of $f=\frac{64}{3 \pi}$ is a reasonable choice.

For this problem it is convenient to re-express Equation 8.3.1 in more "natural" units giving:

$$
M=6.79 \frac{1}{N} \sum_{i=1}^{N}\left(\frac{v_{z i}}{385 \mathrm{~km} / \mathrm{s}}\right)^{2}\left(\frac{R_{i}}{\operatorname{arcmin}}\right)\left(\frac{D}{10 M p c}\right) \times 10^{11} M_{\odot}
$$

where $D$ is the distance to NGC 3115 in tens of Mpc, and $v_{z i}$ is the velocity of the object after removal of the rotational and systemic components.

For the globular clusters around NGC 3115, use of the PME gives the result

$$
M=3.7_{-0.9}^{+1.9} \times 10^{11} M_{\odot} \frac{D}{10 M p c}
$$

where the upper limit assumes that the clusters are on linear orbits and the system has no rotation and is moving with the systemic velocity of the galaxy $(664 \mathrm{~km} / \mathrm{s})$, and the lower limit is derived assuming that the cluster system is rotating, that the systemic velocity is $633 \mathrm{~km} / \mathrm{s}$ and the clusters are on isotropic orbits. 


\subsubsection{The Mass-to-Light Ratio}

As a measure of the level of dark matter within a galaxy we quote here the ratio of mass in units of $M_{\odot}$ to that of luminosity in units of $L_{\odot}$. This ratio reflects the amount of material that is present gravitationally as a multiplier of that which would be present if all the light of a galaxy was derived from stars of solar luminosity. Of interest is the change in $M / L$ as a function of radius. As there should only be minor changes in the composition of the stellar population with radius any changes in the value of $M / L$ with radius reflect a change in the relative importance of non-luminous material.

For the luminosity of NGC 3115 we use $B_{T}^{0}=9.65$ (Capaccioli, Cappellaro, Held, and Vietri 1993); the integrated luminosity contained within the diameter at which the surface brightness of the galaxy falls below $m u_{B}=25.0$ $\left(D_{25}\right)$. This is the same value as that used in the determination of the specific frequency of the GCS. For the value of the solar luminosity we use $M_{B}=5.48$ (Allen 1973). Combining the mass determined above with the luminosity provides a value of

$$
M / L_{B}=19_{-4}^{+10}\left(\frac{D}{10 M p c}\right)^{-1}
$$

Studies of the disk kinematics of NGC 3115 (Capaccioli, Cappellaro, Held, and Vietri 1993) determine that $M / L_{B}\left(R=1^{\prime}\right)=6$ and $M / L_{B}\left(R=3^{\prime}\right) \sim 10$ while we find that $M / L_{B}\left(R=5^{\prime}\right)=19_{-4}^{+10}$. The value of $M / L$ is increasing with distance from the galaxy center, NGC 3115 possess a dark matter halo. The value of $M / L_{B}=20$ is typical of that found using globular cluster velocities of other galaxies and is more typical of spiral than elliptical systems, Table 8.1. 


\subsection{Linking colour with velocity}

A more careful examination of the colour bi-modality is provided if we examine only those objects which have a high likelihood of being globular clusters on the basis of their radial velocities. Figure 8.4 reveals the telling signature of a disk structure in the positions of clusters. Clusters which are redder than the average are confined to the plane of the galaxy disk while those which are blue are distributed spherically about the galaxy center.

A Kolmogrov-Smirnov comparison of the major axis positions of these populations of objects reveals that there is a $60 \%$ probability that they share the same distribution while a comparison of minor axis positions suggests the probability that they are from the same parent population is $\$ 1 \%$. We take this as strong evidence that the two populations have distinct minor axis distributions. This apparent disk structure is reminiscent of that of the Milky Way and further strengthens the suggestion that the GCS of NGC 3115 is a very much like that of a spiral galaxy.

\subsubsection{Rotation}

In section 8.2 a correlation between a cluster's major axis position and its velocity was found. If we now further assert that the blue and red objects come from distinct populations then the rotation curves should be investigated separately. Figure 8.3 presents the radial velocity data from the disk (redder than average) clusters and halo (bluer than average) clusters. From this we see that the disk system of objects is rotating more rapidly than the halo subsystem but both systems have the same mean velocity. For the disk system of clusters the rotation curve approaches an amplitude of $\sim 250 \mathrm{~km} / \mathrm{s}$ at distance of $\sim 3^{\prime}$, a value which is consistent with the asymptotic value of the disk 
TABLE 8.1. Galaxy Mass-to-Light Ratios

\begin{tabular}{rrrll}
\hline \multicolumn{1}{c}{ Name } & Type & M/L & \multicolumn{1}{c}{ radius } & \multicolumn{1}{c}{ Ref. } \\
\hline M31 & SA(s)b & 16 & $R<30 k p c$ & Huchra et al. 1991 \\
M81 & SA(s)ab & 19 & $R<20 k p c$ & Perelmuter et al. 1995 \\
M104 & SA(s)a & 16 & $R<15 k p c$ & Bridges et al. 1997 \\
M49 & E2/S0(2) & $<10$ & $R<20 k p c$ & Mould et al. 1990 \\
NGC 3115 & S0- & 19 & $R<15 k p c$ & This work \\
M87 & E+0-1 pec (cD) & 31 & $R<40 k p c$ & Mould et al. 1990 \\
NGC 1399 & E1 pec & $70-80$ & $20<R<40 k p c$ & Grillmair et al. 1994 \\
\hline \hline
\end{tabular}

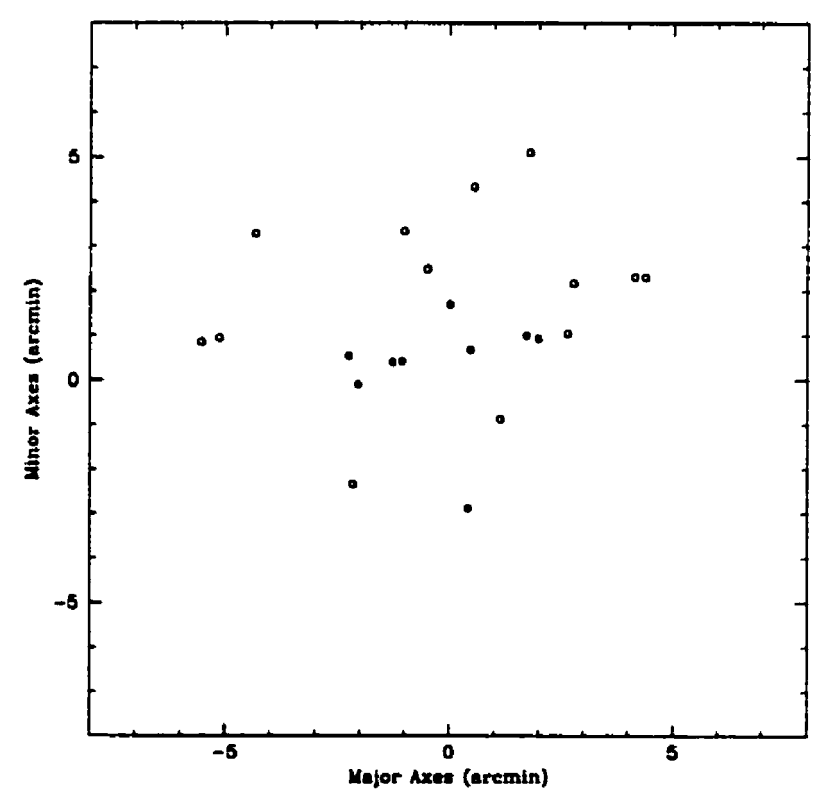

Figure 8.4 Positions of objects selected as being globular clusters on the basis of their radial velocities. Objects with $V-I<1.15$ are shown as open circles while objects with $V-I>1.15$ are shown with filled circles. The coordinates are the projected displacement along the major and minor axis. 
rotation $(v=260 \mathrm{~km} / \mathrm{s}$ (Capaccioli, Cappellaro, Held, and Vietri 1993)). At the same radius the halo system of clusters has a rotation velocity of $\sim 90 \mathrm{~km} / \mathrm{s}$. Although both of these values are very ill-determined (owing to the small number of objects available and the large instrumental uncertainties) both are consistent with the hypothesis that there are two distinct cluster systems present.

\subsubsection{The Mass}

Using fits to the rotation curves for the disk and halo systems alone and then determining the PME reduces the estimated mass by $\sim 40 \%$ and likewise reduces the value of $M / L$. This reduction is caused by the less restricted fit better matching the scatter in the velocity data and thus reducing it by a small fraction. 


\section{Chapter 9}

\section{Discussion and Summary}

This summary examines the properties of the the GCS of NGC 3115 by comparing the GCS of NGC 3115 to the GCSs of other galaxies. Does the lenticular galaxy NGC 3115 appear to be like that of a spiral or that of an elliptical?

\subsection{Spirals}

The GCS of the Milky Way is now understood to be a multiple-component system with at least a thick metal-rich disk of clusters that is distinct from the metal-poor halo of clusters (Zinn 1985). This two-component model is most certainly over simplifying the case, as some MW clusters are now found to be associated with satellite galaxies of the MW (van den Bergh 1994), and the globulars of the inner halo appear to have a luminosity function whose width is distinct from the outer halo clusters, see Appendix B.

The nearest "normal" galaxy, M31, also exhibits disk and halo subsystems of clusters (Huchra, Kent, and Brodie 1991). Again this simple view of a two component family is not complete, with strong evidence that within the halo population there exist sub groups which are dynamically associated and may 
be the remnants of past captures (Ashman and Bird 1993).

\subsection{Ellipticals}

Much effort in the study of GCSs beyond the local group has been expended in the analysis of the cluster systems of elliptical galaxies recently (see (Forbes 1996; Baum, Hammergren, Groth, Ajhar, Lauer, O'Neil, Lynds, Faber, Grillmair, Holtzman, and Light 1995; Fleming, Harris, Pritchet, and Hanes 1995; Kissler, Richtler, Held, Grebel, Wagner, and Capaccioli 1994; Perrett, Hanes, Butterworth, Kavelaars, Geisler, and Harris 1997)). This is most certainly the result of the numerically larger cluster populations that these galaxies possess, making them easier targets for observations. The concentrated analysis of $E$ galaxy GCSs has revealed many interesting features. Ellipticals are found to be more abundant in globular clusters than are spirals (see Figure 6.8) their radial profiles are found to be consistent with those found for spirals (see Figure 6.3) and the peak of the luminosity function of ellipticals maybe somewhat fainter than that of spiral galaxies (Fleming, Harris, Pritchet, and Hanes 1995).

Normal elliptical galaxies can be grouped into two "dynamical" categories. These categories are based on the quadrupole moment of the velocity distribution. "Disky" ellipticals are those galaxies with positive quadrupole moments (ie. somewhat flattened systems) and "boxy" ellipticals have negative quadrupole moments (ie somewhat square shape) (Bender 1988; Nieto and Bender 1989).

Disky ellipticals have cluster specific frequencies that are higher than those of spiral galaxies, possessing nearly twice as many clusters per unit luminosity as spirals (Kissler-Patig 1997). This larger population of clusters may be due to efficient formation of clusters early in the galaxy's formation, a fact that 
would explain the lack of hot gas in these galaxies. In addition the efficient formation of clusters may have inhibited the formation of a large disk thus preventing the formation of a spiral galaxy.

In addition to ellipticals which are over-abundant with respect to spirals there exists a subset (perhaps the dominant group) which have cluster systems that are over-abundant compared to other ellipticals. These higher $S_{N}$ ellipticals are typically drawn from the population of $E$ galaxies referred to as "boxy" ellipticals (Kissler-Patig 1997). This group of galaxies contain hot gas, show little to no evidence of any disk structure, and have isophotal shapes dominated by the velocity dispersion of the member stars with little evidence of rotation (Kormendy and Bender 1996). In addition the radial profiles of the cluster population of this group of galaxies tend to be more flattened then those of "disky" ellipticals (Kissler-Patig 1997). Perhaps the overabundance is related to past mergers of galaxies or the stripping of clusters from their parent galaxies during close encounters (Zepf and Ashman 1993b). Additionally, some ellipticals exhibit spectra and colours that are consistent with the merger formation of globular clusters (Zepf, Carter, Sharples, and Ashman 1995; Hilker and Kissler-Patig 1996).

$\mathrm{cD}$ galaxies are the large dominant ellipticals at the centers of some clusters of galaxies. These galaxies possess extended stellar envelopes and are found at or near the dynamical centers of their host galaxy clusters (Schombert 1988). Some cD galaxies possess a large over-abundance of globular clusters (even after normalizing for luminosity). Perhaps the over-abundance is the result of past merger events or perhaps the central galaxies have come to rest at the center of the cluster's potential where large numbers of previously stripped globulars have settled (West, Cote, Jones, Forman, and Marzke 1995). The 
cluster populations of these galaxies fall off very slowly with radius, even more slowly then boxy ellipticals, and appear to trace the extend light profile of the parent $c D$ galaxy (McLaughlin, Harris, and Hanes 1994). This appears to support the association of the globular clusters with the overall potential of the galaxy cluster rather than that of the galaxy itself.

\subsection{Lenticulars}

Lenticular galaxies have received little attention in the examination of cluster systems (Harris 1996a). Only seven such galaxies have been studied to date and this thesis is the first study to reach past the luminosity peak of the distribution and the first conducted in two band passes. The exact nature of lenticular galaxies is of considerable debate (see for example (van den Bergh 1990)) and so the view taken here is to compare the parameters of the cluster system with those of other galaxy types.

The radial distribution, the peak in the luminosity distribution, the specific frequency of clusters for NGC 3115 and the existence of a disk system of clusters all indicate that this galaxy possesses a GCS that is more like that of a spiral than an elliptical. In addition the $M / L$ ratio of $\sim M / L=20$ is consistent with being intermediate between the values found in spiral galaxies and the $M / L$ found in ellipticals using globular clusters as probes, see Table 8.1. Thus the disky lenticular NGC 3115 appears to be a galaxy whose characteristics are very much like those of typical spiral galaxies, supporting the conclusion that S0 galaxies are an extension of the spiral galaxy type towards that of disky ellipticals and not associated with boxy ellipticals (Kormendy and Bender 1996).

What does the GCS of NGC 3115 tell us about its formation? Clearly the 
multiple components (in metallicity) tell of a episodic formation. The metalrich system is concentrated towards the center of the galaxy while the more metal-poor objects lie at larger radii. In addition the dynamics of the two systems are distinguishable favoring an accretion model of formation for this galaxy. These clues to the past of NGC 3115 are consistent with the discovery of two families of red giant branch stars in the halo of NGC 3115 (distinguished by their metallicity).

\subsection{Summary}

The main conclusions of this thesis are as follows:

- The GCS of NGC 3115 is at least a bi-modal system of objects; one system is dynamically, spatially and by stellar content associated with the disk of the galaxy and the other associated with the halo. This is typical of spiral systems (M31 \& MW).

- NGC 3115 possesses a dark matter halo; the $M / L$ value rises with increasing distance from the galactic center and reaches a value of $M / L=$ $19_{-4}^{+10}$. This value of $M / L$ is more typical of elliptical systems then that of spirals.

- The specific frequency of globular clusters, the shape and peak height of the GCLF and the radial profile of clusters are all reminiscent of spiral systems.

- NGC 3115 appears, by its cluster population, to be intermediate between spiral and disky elliptical in its type. 


\section{Bibliography}

Abraham, R. G. \& van den Bergh, S. 1995, January. ApJ, 438, 218-222.

Allen, C. W. 1973. In London: University of London, Athlone Press, $-c 1973,3 r d$ ed.

Ashman, K. M. \& Bird, C. M. 1993, December. AJ, 106, 2281-2290.

Ashman, K. M., Conti, A., \& Zepf, S. E. 1995, September. AJ, 110, 1164+.

Babcock, H. W. 1939. Lick Obs. Bull, 19, 41-51 (1939), 19, 41-51.

Bachall, J. N. \& Scott, T. 1981, March. ApJ, 244, 805-819.

Baum, W. A., Hammergren, M., Groth, E. J., Ajhar, E. A., Lauer, T. R., O'Neil, E. J., J., Lynds, C. R., Faber, S. M., Grillmair, C. J., Holtzman, J. A., \& Light, R. M. 1995, December. AJ, 110, $2537+$.

Bender, R. 1988, March. A\&A, 193, L7-L10.

Blakeslee, J. P. \& Tonry, J. L. 1995, April. ApJ, 442, 579-588.

Bridges, T. J., Ashman, K. M., Zepf, S. E., Carter, D., Hanes, D. A., Sharples, R. M., \& Kavelaars, J. J. 1997, January. MNRAS, 284, 376384.

Brodie, J. P. \& Hanes, D. A. 1986, January. ApJ, 300, 258-278.

Brodie, J. P. \& Huchra, J. P. 1991, September. ApJ, 379, 157-167.

Butterworth, S. T. 1997. Private communication. Simmulations conducted in software testing, unpublished.

Capaccioli, M., Cappellaro, E., Held, E. V., \& Vietri, M. 1993, July. A\&A, $274,69+$.

Capaccioli, M., Vietri, M., \& Held, E. V. 1988, September. MNRAS, 234, 335-340.

Carlberg, R. G., Yee, H. K. C., Ellingson, E., Abraham, R., Gravel, P., Morris, S., \& Pritchet, C. J. 1996, May. ApJ, 462, 32+. 
Castellani, V. \& Melchiorri, M. 1981, December. Ap\&SS, 80, 289-302.

Davies, R. L. 1981, March. MNRAS, 194, 879-902.

de Vaucouleurs, G., de Vaucouleurs, A., Crowin Jr, H., Buta, R. J., Paturel, G., \& Fouque, P. 1991. Third Reference Catalogueof Bright Galaxies (3.9 ed.). Springer, New York.

Eggen, O. J., Lynden-Bell, D., \& Sandage, A. R. 1962, November. ApJ, 136, $748+$.

Elson, R. A. W. 1997, April. MNRAS, 286, 771-776.

Fernie, J. D. 1962, December. AJ, 67, 769+.

Fischer, P., Hesser, J. E., Harris, H. C., \& Bothun, G. D. 1990, January. PASP, 102, 5-25.

Fischer, P., Welch, D. L., Mateo, M., \& Cote, P. 1993, October. AJ, 106, 1508-1523. Provided by the NASA Astrophysics Data System.

Fleming, D. E. B., Harris, W. E., Pritchet, C. J., \& Hanes, D. A. 1995, March. AJ, 109, 1044-1054.

Forbes, D. A. 1996, September. AJ, 112, 954t.

Freeman, K. 1996. Notes on the Kinematics of Spiral Galaxies, presented at the 1996 CASCA AGM. Private Bag, Weston Creek, ACT 2611, Australia: MSSSO.

Grillmair, C. J., Freeman, K. C., Bicknell, G. V., Carter, D., Couch, W. J., Sommer-Larsen, J., \& Taylor, K. 1994, February. ApJ, 422, L9-L12.

Hanes, D. A. 1977, May. MNRAS, 179, 331-337.

Hanes, D. A. \& Brodie, J. P. 1986, January. ApJ, 300, 279-288.

Hanes, D. A. \& Harris, W. E. 1986, May. ApJ, 304, 599-606.

Hanes, D. A. \& Whittaker, D. G. 1987, October. AJ, 94, 906-916.

Harris, G. L. H., Geisler, D., Harris, H. C., \& Hesser, J. E. 1992, August. AJ, 104, 613-626. Provided by the NASA Astrophysics Data System.

Harris, W. 1996a, March. Globular cluster systems in other galaxies. http://www.physics.mcmaster.ca/GC/s_n.dat.

Harris, W. E. 1976, December. AJ, 81, 1095-1116.

Harris, W. E. 1986, April. AJ, 91, 822-841.

Harris, W. E. 1991. ARA\&A, 29, 543-579. 
Harris, W. E. 1996b, October. AJ, 112, 1487+.

Harris, W. E. \& Van Den Bergh, S. 1981, November. AJ, 86, 1627-1642.

Hilker, M. \& Kissler-Patig, M. 1996, October. A\&A, 314, 357-367.

Horne, K. 1986, June. PASP, 98, 609-617.

Huchra, J. P., Kent, S. M., \& Brodie, J. P. 1991, April. ApJ, 370, 495-504.

Illingworth, G. \& Schechter, P. L. 1982, May. ApJ, 256, 481-496.

Jacoby, G. H., Branch, D., Clardullo, R., Davies, R. L., Harris, W. E., Pierce, M. J., Pritchet, C. J., Tonry, J. L., \& Welch, D. L. 1992, August. PASP, 104, 599-662.

Jones, L. A. \& Worthey, G. 1995, June. ApJ, 446, L31-t.

Kissler, M., Richtler, T., Held, E. V., Grebel, E. K., Wagner, S. J., \& Capaccioli, M. 1994, July. A\&A, 287, 463-469.

Kissler-Patig, M. 1997, March. A\&A, 319, 83-91.

Kormendy, J. \& Bender, R. 1996, June. ApJ, 464, L119-+.

Kormendy, J., Bender, R., Richstone, D., Ajhar, E. A., Dressler, A., Faber, S. M., Gebhardt, K., Grillmair, C., Lauer, T. R., \& Tremaine, S. 1996, March. ApJ, 459, L57-+.

Kormendy, J. \& Richstone, D. 1992, July. ApJ, 393, 559-578.

Larson, R. B. 1990, July. PASP, 102, 709-722.

Mathews, G. J., Kajino, T., \& Orito, M. 1996, January. ApJ, 456, 98+.

McLaughlin, D. E., Harris, W. E., \& Hanes, D. A. 1994, February. ApJ, 422, 486-507.

Merritt, D. \& Tremblay, B. 1993, December. AJ, 106, 2229-2242.

Mihalas, D. \& Binney, J. 1981. In San Francisco, CA, W. H. Freeman and Co., 1981. 608 p., pp. 53...+.

Morton, D. C. \& Chevalier, R. 1973. ApJ, 179, 55-68.

Mould, J. R., Oke, J. B., De Zeeuw, P. T., \& Nemec, J. M. 1990, June. AJ, $99,1823-1830$.

Mould, J. R., Oke, J. B., \& Nemec, J. M. 1987, January. AJ, 93, 53-59.

Murali, C. \& Weinberg, M. D. 1997, June. MNRAS, 288, 749-766.

Nieto, J. L. \& Bender, R. 1989, May. A\&A, 215, 266-271. 
Novotny, E. 1973. In New York: Oxford University Press, 1973.

Oort, J. H. 1940, april. ApJ, 91, 273-306.

Peebles, P. J. E. 1993. In Princeton, N.J. : Princeton University Press, c1993.

Perelmuter, J.-M., Brodie, J. P., \& Huchra, J. P. 1995, August. AJ, 110, $620+$.

Perrett, K. M., Hanes, D. A., Butterworth, S. T., Kavelaars, J., Geisler, D., \& Harris, W. E. 1997, March. AJ, 113, 895+.

Porter, A., Davis, L., \& Hall, P. 1990. Iraf/PHOTCAL catalogs. KPNO.

Rees, M. J. 1984, December. Journal of Astrophysics and Astronomy (ISSN 0250-6335), vol. 5, Dec. 1984, p. 331-348., 5, 331-348.

Rodgers, A. W. \& Paltoglou, G. 1984, August. ApJ, 283, L5-L7.

Rubin, V. C. 1983, June. Science (ISSN 0036-8075), vol. 220, June 24, 1983, p. 1339-1344., 220, 1339-1344.

Rubin, V. C. 1995, October. ApJ, 451, 419+.

Rubin, V. C., Peterson, C. J., \& Ford, W. K., J. 1980, July. ApJ, 239, 50-53.

Schombert, J. M. 1988, May. ApJ, 328, 475-488.

Searle, L. \& Zinn, R. 1978, October. ApJ, 225, 357-379.

Secker, J. 1992, October. AJ, 104, 1472-1481.

Secker, J. \& Harris, W. E. 1993, April. AJ, 105, 1358-1368.

Shapley, H. 1918. ApJ, 48, 154.

Stetson, P. B. 1987, March. PASP, 99, 191-222.

Strom, K. M., Strom, S. E., Jensen, E. B., Moller, J., Thompson, L. A., \& Thuan, T. X. 1977, March. ApJ, 212, 335-337.

Tonry, J. \& Davis, M. 1979, October. AJ, 84, 1511-1525.

Van Den Bergh, S. 1969. ApJS, 19, 145-174.

van den Bergh, S. 1990, January. ApJ, 348, 57-60.

van den Bergh, S. 1994, December. AJ, 108, 2145-2153. Provided by the NASA Astrophysics Data System.

van den Bergh, S. 1997, January. AJ, 113, 197+.

Walker, M. F. 1989, April. PASP, 101, 333-350. 
West, M. J., Cote, P., Jones, C., Forman, W., \& Marzke, R. O. 1995, November. ApJ, 453, L77-+.

Zepf, S. E. \& Ashman, K. M. 1993a, January. In NASA. Ames Research Center, The Evolution of Galaxies and Their Environment $p$ 48-49 (SEE N93-26706 10-90), pp. 48-49.

Zepf, S. E. \& Ashman, K. M. 1993b, October. MNRAS, 264, 611+.

Zepf, S. E., Carter, D., Sharples, R. M., \& Ashman, K. 1995, May. ApJ, 445, L19-L22.

Zinn, R. 1985, June. ApJ, 293, 424-444.

Zinn, R. \& West, M. J. 1984, May. ApJS, 55, 45-66.

Zwicky, F. 1933. Helvetica Physica Acta, 6, 100. 


\section{Appendix A}

\section{Acronyms}

CCD charge coupled device An electronic semi-conductor based photon detection device. Photons striking the surface of the semiconductor substrate cause electrons in the substrate to become energized. These electrons are then captured into potential wells in the semi-conductor material.

CFHT Canada-France-Hawaii Telescope. The Canada-France-Hawaii Telescope is operated by the National Research Council of Canada, the Centre National de la Recherche Scientifique de France and the University of Hawaii.

CTIO Cerro Tololo Inter-American Observatory, National Optical Astronomy Observatories, operated by the Association of Universities for Research in Astronomy (AURA), Inc., under contract with the National Science Foundation.

DM dark matter This term refers generically to all forms of matter that are non-luminous in the optical. eg. toasters, buses, Jupiter type planets, black holes, and this thesis.

GC globular cluster. A tightly bound, self-gravitating, group of $\sim 10^{5}$ population II stars.

GCLF globular cluster luminosity function. The integrated luminosity function of a GCS. NOT the luminosity function of an individual GC.

GCS globular cluster system. The population of globular clusters associated with a galaxy. 
LDSS low dispersion survey spectrograph. The MOS system at the WHT.

MOS multi-object spectrograph A spectrographic device designed to allow the user to acquire the spectra of multiple objects within the same field of view.

MW Milky Way The galaxy in which the solar system is embedded.

PME projected mass estimator An estimate of the mass of body based on the projected velocities of objects in orbit about the body. This estimated is based on the virial theorem with weighting for the radius of the orbit of the test body included.

PSF point-spread-function The blurring of point-like images caused by the Earth's atmosphere and the optics of the telescope and detector system.

QE quantum efficiency The number of photons detected by a CCD as a percent of the number of photons striking the device.

SBB standard Big Bang. The currently accepted standard model of the early stages of the evolution of the Universe.

WHT The William Herschel Telescope. The WHT is operated on the island of La Palma by the Royal Greenwich Observatory at the Spanish Observatorio del Roque de los Muchachos of the Instituto de Astrofisica de Canarias. 


\title{
Appendix B
}

\section{A comparison of the globular cluster luminosity functions of the inner and outer halo of the Milky Way and M31.}

\author{
JJ Kavelaars and D.A. Hanes \\ Department of Physics, Queen's University \\ Kingston, ON K7L 3N6 (CANADA)
}

\section{B.1 Abstract}

We show that the globular cluster luminosity function (GCLF) of the inner halo of the Milky Way is statistically different from the GCLF of the outer halo. We also find a similar difference between the inner and outer halo globular cluster populations of M31. We assert that this difference is evidence for some form of dynamical evolution of the cluster population and/or a dependence of GCLF shape on the environment in which the cluster population formed. We also find that the turnover luminosity of the GCLF is unaffected by these differences and further assert that this stability of the turnover luminosity affirms its usefulness as an indicator of cosmic distance. 


\section{B.2 Introduction}

The globular cluster system of the Milky Way has long been known to be a multiple component system that can be divided into disk and halo subsystems; in addition, the halo subsystem can be further divided into an inner and outer halo (Searle and Zinn 1978). This division of the halo into inner and outer components has allowed the discrimination of the clusters along age boundaries on the basis of horizontal branch type (Lee et. al 1994). For an excellent review of these issues see Zinn (1996) and other works in the same volume.

There is an emerging acceptance of the view that the current population of globulars is not the primordial distribution but rather the surviving component of a once grander population (Fall and Rees 1977; Murali and Weinberg 1996a). This view is directly testable within the Milky Way globular cluster population itself: it has been suggested (Murali and Weinberg 1996a) that outer halo clusters will not suffer significant disruption while those which are interior to $R_{G C} \sim 8 k p c$ do, with consequential changes in the observed GCLFs.

Given both that the outer halo clusters are younger and that their orbits make them less likely to have undergone externally imposed dynamical evolution, the outer halo cluster population should be most like that of a primordial system while that of the inner halo should be evolved. This reasonable view can be tested by a comparison of the globular cluster systems (hereafter GCSs) of the inner and outer halo. To allow an extension of this comparison to external galaxies, the comparison should be between observables which can be studied in remote systems. The globular cluster luminosity function (GCLF) is the obvious choice.

\section{B.3 The Milky Way GCLF, inside and out}

Figure B.1 shows (a) the GCLF for all of the halo clusters $([\mathrm{Fe} / \mathrm{H}]<-0.8)$ of the Milky Way, and the GCLFs for the inner $\left(R_{G C}<8 \mathrm{kpc}\right)(\mathrm{b})$ and outer $\left(R_{G C}>8 \mathrm{kpc}\right.$ ) (c) halo subsystems. (These data were taken from the McMaster University globular cluster database maintained by W.E. Harris ${ }^{1}$ ). There are approximately $\mathbf{5 0}$ clusters in each of the subdivisions and so the differences between these two distributions are decidedly real. A Kolmogrov-Smirnov comparison of the two populations gives a probability of less than $5 \%$ that the two distributions come from the same parent population. This evidence

\footnotetext{
${ }^{\text {' }}$ http://www.physics.mcmaster.ca/Globular.html
} 
strongly suggests that the inner and outer halo populations have different formation histories and/or different evolutionary histories.

Fits of the function

$$
A \exp \left(-\left(m-M_{T O}\right)^{2} /\left(2 \sigma^{2}\right)\right)
$$

to the three distributions are overlaid on the histograms in Figure B.1, with the numerical results shown in Table B.1. These fits were performed using the NGAUSSFIT routine in STSDAS under the assumption of Poisson sampling errors. It is of interest to note that although the inner and outer halo GCLFs have markedly different distributions, they have the same peak luminosity.

Parenthetically, we acknowledge that the Gaussian representation is not an optimal fit to the GCLF in the outer sample in particular. We use it partly because of its long standing as a tool in extra-galactic distance determinations (Hanes 1977; Harris 1991), but emphasis the important point that a variety of statistical descriptors demonstrate that the mean luminosities in the two sub-samples are not significantly different. A Kolmogrov-Smirnov test, however, reveals that there is a statistically significant probability that the two samples are not drawn from the same parent population: the shapes are clearly different.

These results together appear to be in conflict with recent dynamical models (Murali and Weinberg 1996a) which suggest that the peak value of the GCLF will become significantly fainter as the cluster population evolves, and that such evolution should be strongest for the inner halo population. It appears, then, that a model is required in which the dynamical erosion of bright and faint clusters occurs indifferently. ${ }^{2}$

\section{B.4 The GCS of M31}

The recognition of the two-component nature of the Milky Way halo cluster luminosity function immediately suggests an examination of other GCLFs under the same conditions. The obvious choice for such a comparison is M31. To allow such a comparison it is necessary to divide the M31 cluster population into disk and halo subsystems. The question of which M31 clusters are halo members and which are disk members is confused by the lack of spectroscopic metallicities for nearly half the cluster candidates. Ideally a cut of metallicity similar to that used for the Milky Way would isolate the disk and halo

\footnotetext{
${ }^{2}$ Recent additional simulations (Murali and Weinberg 1996b) are consistent with the findings we report here.
} 

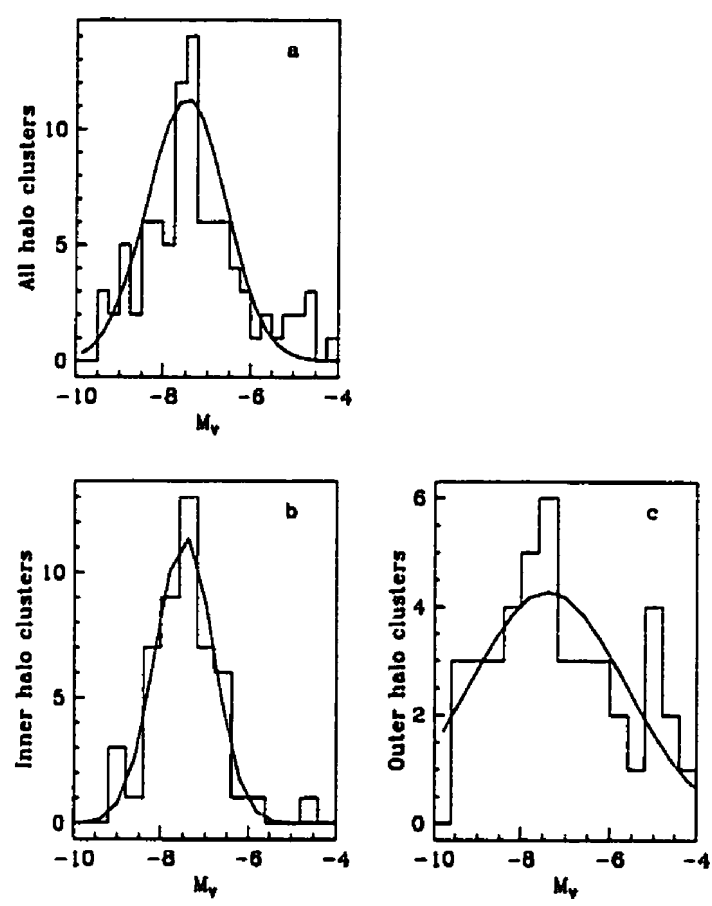

Figure B.1 (a) The luminosity function for the halo globular clusters of the Galaxy. (b) The luminosity function for the inner halo clusters. (c) The luminosity function for the outer halo clusters. A Kolmogrov-Smirnov test shows that there is a $3.7 \%$ probability that the two distributions are drawn from the same parent population (ie. the hypothesis that these two distributions are from the same parent population is rejected).

Table B.1 Fits to the GCLFs for various components of the Milky Way GCS. These fits were performed using the NGAUSSFIT package in STSDAS. The data are from the McMaster catalogue. Note that the peak or turnover magnitude remains constant (within uncertainties) for all components in the Galaxy but the outer halo population is significantly broader.

$\begin{array}{cccc}\text { MW-GCS } & M_{\text {TO }} & \sigma_{\text {GCLF }} & \mathrm{N} \\ \text { all clusters } & -7.44 \pm 0.15 & 1.08 \pm 0.1 & 132 \\ \text { all halo clusters } & -7.48 \pm 0.15 & 0.90 \pm 0.1 & 93 \\ \text { inner halo clusters } & -7.47 \pm 0.13 & 0.66 \pm 0.1 & 49 \\ \text { outer halo clusters } & -7.41 \pm 0.4 & 1.76 \pm .3 & 44\end{array}$


subsystems, but in practice such a straightforward approach is not possible. However, there exists a strong correlation between the integrated colours and metallicities of globular clusters (Reed et. al 1994b). It is possible then to make a reasonable separation of M31 GCS into disk and halo populations on the basis of colour.

Previous authors have selected clusters as halo members based on their position on the sky (Reed et. al 1994a). This selection criterion has the disadvantage of preferentially excluding many inner halo clusters. To avoid such a bias, we select as our "halo sample" all clusters for which $(B-V)<0.8$ in the Battistini et al. (1987) survey. This selection seems justified in that for the Galaxy there are no disk clusters bluer than $(B-V)=0.8$ and so this cut should eliminate the majority of disk clusters from the M31 sample. Unfortunately this selection criterion also removes many true halo members from the sample; however, we find that there is no significant difference in parameters between a Milky Way halo sample selected as $[\mathrm{Fe} / \mathrm{H}]<-0.8$ and one selected using $(B-V)<0.8$. This culling also has the advantage of excluding background galaxies from the Battistini et al. (1987) A,B sample (see (Reed et. al 1994b) Figure 8).

In panel (a) of Figure B.2 we present the LF for all the globular cluster candidates which have (B-V) $<0.8$ (from Battistini (1987)). In panel (b), we present the luminosity function for the inner halo objects, that subset of objects which lie within a projected radius of $10 \mathrm{kpc}(140 \mathrm{arcmin}$ for a true distance modulus of 24.45 (Jacoby et al. 1992)) of the center of M31; in panel (c) we show the luminosity function for the objects beyond $10 \mathrm{kpc}$. This figure qualitatively reveals the same separation of the GCLF into peaked and flat components, when selected on the basis of radius, as was seen for the Galaxy. (The widths $(\sigma)$ of the fitted Gaussians differ formally by $\sim 2.3$ times the combined uncertainties.) We do not, however, consider this as strong a test as that afforded by the MW sample because of the uncertain extent to which incompleteness affects the luminosity functions in the subsamples at the faint end. In addition, of course, there will be some contamination of the "inner halo" subsample by outer halo clusters projected into the $10 \mathrm{kpc}$ circle. Also, the determination of halo/disk membership for individual clusters is uncertain and it is probable that there is contamination of the inner halo sample by disk clusters.

Table B.2 presents the results of fits of equation B.1 to the various cluster populations in M31. As was found for the Milky Way GCS the outer halo clusters of M31 have a broader luminosity function, and once again there is no 

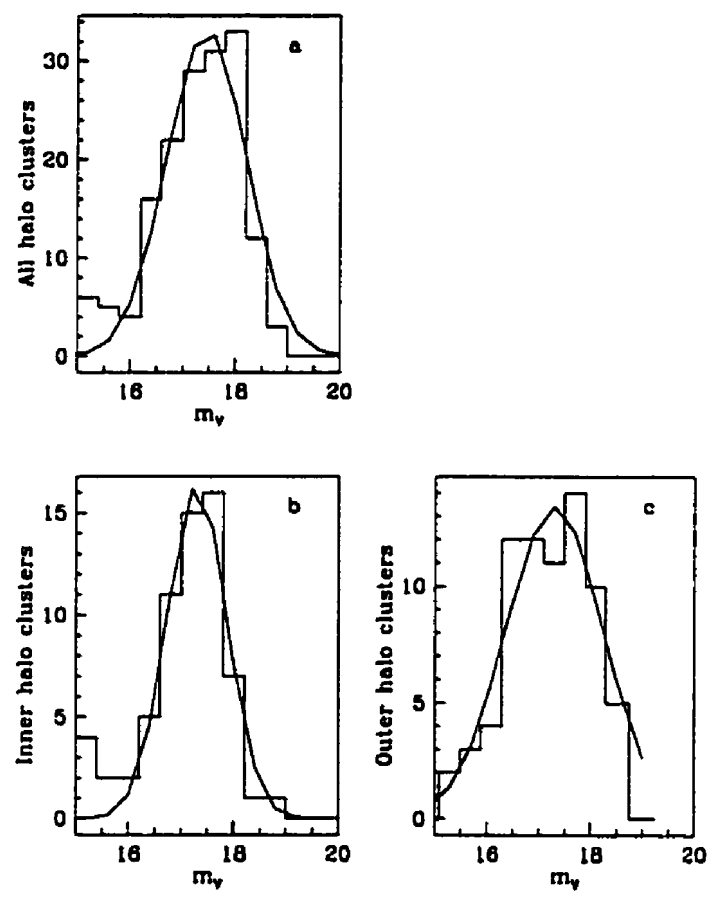

Figure B.2 (a) The luminosity function for the halo globular clusters of the M31. (b) The GCLF for the inner halo clusters. (c) The GCLF for the outer halo clusters. A Kolmogrov-Smirnov test shows that there is a $18 \%$ probability that the two distributions (inner verses outer) are drawn from the same parent population (ie. the hypothesis that these two distributions are from the same parent population is not supported). 
evidence for a difference between the mean luminosity of the clusters in the inner and outer halo. A K-S comparison of the data sets gives a probability of $18 \%$ that the two samples are drawn from the same parent population. This provides marginal evidence for a multiple component halo and is consistent with previous work (Ashman and Bird 1993; Huchra et al. 1991) which has shown that the distribution of halo clusters in M31 contains spatial substructure.

As for the Milky Way, the observed difference between the two M31 halo populations cannot be the result of dynamical evolution of the type predicted by Murali and Weinberg (1996) since their model implies that the inner population should appear measurably fainter than that of the outer. There is no evidence for such a shift in mean luminosities.

\section{B.5 Conclusions}

Both within the Milky Way and, to a lesser extent, in M31 the outer halo clusters are clearly more indicative of a broad luminosity function. This population may be, then, indicative of a broad initial mass function for globular clusters.

The inner halo clusters demonstrate a remarkably peaked distribution. The difference between the inner and outer luminosity functions may indicate the fate that dynamical evolution has in store for clusters formed near the centers of galaxies.

The turnover luminosities of the inner and the outer cluster populations appear to be consistent. If this is the case, then dynamical models of cluster evolution will need to account for the preferential stripping away of clusters fainter and brighter than the turnover luminosity. This decoupling of mean luminosity from position in the galaxies gives further assurance that GCLFs

Table B.2 Fits to the GCLFs for various components of the Andromeda galaxy (M31). These fits were performed using the NGAUSSFIT package in STSDAS. The data are from Battistini et. al (1987). Once again, the outer halo population is somewhat broader than that of the inner halo sub-system.

$\begin{array}{cccc}\text { M31-GCS } & m_{T O} & \sigma_{G C L F} & \mathrm{~N} \\ \text { all halo clusters } & 17.45 \pm 0.08 & 0.76 \pm 0.05 & 161 \\ \text { inner halo clusters } & 17.30 \pm 0.10 & 0.57 \pm 0.10 & 64 \\ \text { outer halo clusters } & 17.29 \pm 0.17 & 0.94 \pm 0.13 & 97\end{array}$


can be used as standard candles in cosmic distance determinations.

The dependence of the shape of the GCLF on galactocentric radius, however, suggests that future comparisons should only be made between cluster populations that include clusters at comparable distances from their host galaxy centers. If cluster disruption depends on the mass of the parent galaxy and if the form of the GCLF is the result of dynamical evolution, the severity of the effect will depend on galactocentric distance and galaxy mass. Determining what cluster sub-populations are comparable then becomes a question which is model dependent.

If dynamical effects are not found to be responsible for the dependence of GCLF shape on radius, then the environment in which clusters form is likely to be the deciding factor.

Many thanks to S.T. Butterworth and D. Wing for their clarifying discussion of this issue. We also thank the referees for their helpful comments on many aspects of this manuscript.

\section{B.6 References}

Ashman, K.M., Bird, C.M. 1993 AJ 106, 2281

Battistini, P., Bonoli, F., Braccesi, A. Federici, F. Fusi Pecci, F., Marano, B., Borngen, F., 1987, A\&AS, 67, 447

Fall, S.M., Rees, M.J. 1977, MNRAS, 181, 37P

Hanes, D.A. 1977, MNRAS, 180, 309

Harris, W.E. 1991, ARAA, 29, 543

Huchra, J.P., Brodie, J.P., Kent, S.M., 1991, ApJ, 370, 495

Jacoby, G.H. et al., 1992, PASP, 104, 599

Lee, Y.-W., Demarque, P., Zinn, R., ApJ, 423, 248

Murali, C., Weinberg, M.D., 1996a, MNRAS, submitted; preprint astro-ph/9604049

Murali, C., Weinberg, M.D., 1996b, MNRAS, submitted; preprint astro-ph/9610229

Reed, L.G., Harris, G.L.H., Harris, W.E., 1994a, AJ, 103, 824

Reed, L.G., Harris, G.L.H., Harris, W.E., 1994b, AJ, 107, 555 
Searle, L., Zinn, R., 1978, ApJ, 225, 357

Secker, J., 1992, AJ, 104, 1472

Zinn, R., 1996, ed. H. Morrison and A. Sarajedini, ASP Conf. Ser. 92, Formation of the Galactic Halo. . . .Inside and Out, 211 
